\title{
Combining force and fluorescence microscopy for the manipulation and detection of single cells, viruses, and proteins
}

\author{
Dissertation \\ for the award of the degree \\ "Doctor rerum naturalium" \\ of the Georg-August-Universität Göttingen
}

within the doctoral program

Physics of Biological and Complex Systems

of the Georg-August University School of Science (GAUSS)

\author{
submitted by \\ Kai Bodensiek \\ from Wolfenbüttel \\ Göttingen, August 31, 2014
}




\title{
Members of the Thesis Committee:
}

\author{
Dr. Iwan A.T. Schaap (Supervisor, 1st Reviewer) \\ Atomic Force Microscopy Group at the Third Institute of Physics \\ Faculty of Physics \\ Prof. Dr. Andreas Janshoff (2nd Reviewer) \\ Institute for Physical Chemistry \\ Faculty of Chemistry \\ Prof. Dr. Helmut Grubmüller \\ Director of Max Planck Institute for Biophysical Chemistry
}

Additional Members of the Examination Committee:

\author{
Prof. Dr. Kai Tittmann \\ Albrecht-von-Haller Institute \\ Faculty of Biology and Psychology \\ PD Dr. Peter Dechent \\ MR Research / Department of Cognitive Neurology \\ University Medicine \\ Dr. Dieter Klopfenstein \\ Third Institute of Physics \\ Faculty of Physics
}




\section{Contents}

1 Outline of this thesis 6

2 Introduction 8

2.1 Atomic Force Microscopy . . . . . . . . . . . . . . . . 8

2.1.1 Cantilever calibration . . . . . . . . . . . . . 10

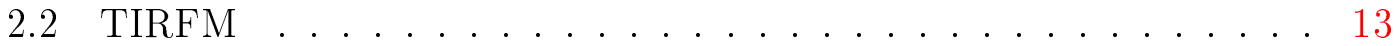

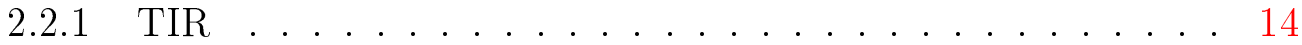

2.2 .2 TIR excitation . . . . . . . . . . . . . 17

2.3 Optical Tweezers . . . . . . . . . . . . . . . . . . 19

2.3.1 Trapping forces and the ray optics model . . . . . . . . 20

2.3.2 Trap calibration . . . . . . . . . . . . . . . . 21

2.4 Biological samples . . . . . . . . . . . . . . . . . 24

2.4.1 Cell mechanics and Parkinson's Disease associated LRRK2

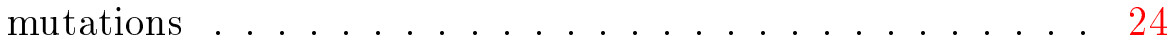

2.4.2 Adenovirus infection pathway and structure . . . . . . . 25

$2.4 .370 \mathrm{~S}$ ribosome . . . . . . . . . . . . . 28

2.4.4 Actin-binding protein DBN-1 . . . . . . . . . . 29

3 Methods and materials 31

3.1 TIRFM beam path design . . . . . . . . . . . . . 31

3.2 Localization microscopy . . . . . . . . . . . . . 35

3.3 Fluorescence experiments with single labeled ribosomes . . . . . . 37

3.3.1 Cover glass functionalization and buffer . . . . . . . . . . 37

3.4 Optical tweezers . . . . . . . . . . . . . . . . . 38

3.4.1 Beam path design . . . . . . . . . . . . . . 39

3.4.2 Optical tweezers alignment . . . . . . . . . . . 42

3.4.3 Sample chamber and beads . . . . . . . . . . . . 42 
3.4.4 Fitting the periodicity of the interference effect between bead and coverslip ..................... 43

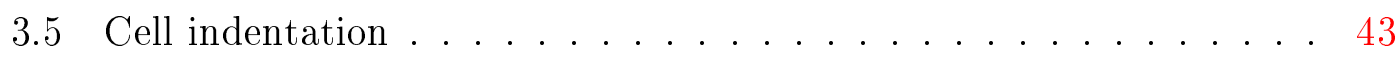

3.5.1 Mechanical description of bio material . . . . . . . . 43

3.5.2 Contact mechanics . . . . . . . . . . . . . . 45

3.6 Mechanical Adenovirus unpacking . . . . . . . . . . . . . . . . 48

3.6.1 Determining genome spread after capsid breaking . . . . . 48

3.6.2 Sample dilution and dye concentration . . . . . . . . . 50

3.6.3 Sample support and immobilization . . . . . . . . . 50

3.6.4 AFM and TIRFM detection . . . . . . . . . . . 52

4 Results and Discussion $\quad 54$

4.1 The combined TIRFM-AFM setup . . . . . . . . . . 54

4.1 .1 Design ....................... 54

4.1 .2 Noise uncoupling . . . . . . . . . . . . 56

4.1.3 Controlling the depth of the evanescent field . . . . . . . 58

4.1.4 TIRFM localization accuracy . . . . . . . . . . 59

4.1.5 TIR excitation decay . . . . . . . . . . . . 65

4.1 .6 Discussion . . . . . . . . . . . . . . . 66

4.2 Single molecule fluorescence with TIRFM-AFM . . . . . . . . 67

4.2.1 Introduction to the experiments . . . . . . . . 67

4.2 .2 Bleaching times . . . . . . . . . . . 67

4.2.3 Achievable frame rates . . . . . . . . . . . . . 72

4.2.4 Background light from AFM cantilevers during single molecule detection . . . . . . . . . . . . . . 73

4.2.5 Fluorescence quenching by AFM tips . . . . . . . . . 76

4.2 .6 Disscussion . . . . . . . . . . . . . . . . . 77

4.3 Practical AFM force set point limits in liquid environments . . . . . 78

4.4 The optical tweezers setup . . . . . . . . . . . . . . . 81

4.4.1 Opto-mechanical design . . . . . . . . . . . 82

4.4 .2 Force limits . . . . . . . . . . . . . . . . . . . 84

4.4.3 Trap stiffness in vertical direction . . . . . . . . . . 87

4.4.4 Interferometry between the trapped bead and the surface . . 88

4.4 .5 Focus shift . . . . . . . . . . . . . 90

4.4 .6 Discussion . . . . . . . . . . . . . . . . 91 
4.5 The application of the AFM-TIRFM setup on biological samples . . 93

4.5.1 Cell mechanics and Parkinson's Disease associated LRRK2 mutations . . . . . . . . . . . . . 93

4.5.2 Actin binding protein DBN-1 . . . . . . . . . 98

4.5.3 Mechanical unpacking of Adenovirus capsids . . . . . . . . . 101

$\begin{array}{llr}5 \text { Conclusion } & 116\end{array}$

6 Appendix 119

6.1 Mechanical unpacking of Adenovirus capsids: supplementary data . 120

6.2 Custom programs . . . . . . . . . . . . . . . . . 122

$\begin{array}{llr}7 & \text { List of abbreviations } & 129\end{array}$

$\begin{array}{ll}\text { List of Tables } & 131\end{array}$

$\begin{array}{ll}\text { List of Figures } & 132\end{array}$

$\begin{array}{ll}\text { Bibliography } & 135\end{array}$ 


\section{Outline of this thesis}

Total Internal Reflection Fluorescence Microscopy (TIRFM) and Atomic Force Microscopy (AFM) are both successfully applied in biophysics and biology. AFM provides the possibility to image and to mechanically manipulate the samples on a nanometer scale. TIRFM allows the observation of single fluorescent molecules close to the surface at a high signal-to-noise ratio and, consequently, improves the temporal and spatial localization of events of interest. The ability to use both microscopy techniques simultaneously allows to study specific biochemical changes (by using fluorescence markers) that are induced by mechanical stimulation (AFM) and gives access to new methodical approaches.

This thesis describes the construction of a combined TIRFM-AFM instrument, its possibilities, and limitations, e.g. time correlated mechanical manipulation and fluorescence detection, effects of the tip inside the excitation and emission volume, as well as noise interactions of both systems. To investigate the tip effects, the fluorescence of single dye molecules linked to ribosomes was characterized. The background light from the AFM cantilevers during single molecule detection was determined as well as the quenching potential of the tip.

The TIRFM-AFM setup was applied to investigate biological samples from the micrometer length scale of living cells down to the nanometer length scale of viruses and actin protein filaments.

1) On the cell scale the TIRFM capabilities were used to identify three sub-types of a Parkinson-like cell line in a mixed population which could not be separated by cell-biological procedures. After identification, the AFM was applied to probe the mechanical properties of the individual sub-types. The results indicate a sub-type specific interaction between the fluorescently labeled Leucine-rich repeat kinase2 (LRRK2) and parts of the cytoskeleton which lead to a different mechanical response for each of the sub-types. 
2) On the virus scale AFM-induced mechanical fatigue experiments were performed on the Adenovirus. The goal was to disassemble the virus capsid and to visualize the release of the genome in real time by specific fluorescence labeling of Deoxyribonucleic acid (DNA). We compared the genome release of the wild type mature adenovirus with that of a non-infectious immature-like mutant. The measurements show that the immature genome remains compact after the shell is opened up by AFM. This suggests that the maturation step is essential for rendering the virus to be infective.

3) On the protein level we studied a newly discovered protein that is involved in the organization of the actin-myosin fibers in muscle cells of C. elegans. In vitro, the protein called DreBriN-like family homolog 1 (DBN-1) induces bundling of actin filaments. These bundles tend to form loops. TIRFM allowed to target these bundles and loops by fluorescence labeling of actin. Subsequent high resolution AFM imaging revealed a decoration of the actin filaments with the 'cross-linking' DBN-1 proteins.

As an additional project an 'optical AFM' was developed. This instrument is based on optical tweezers and specifically designed to overcome the noise limitations of AFM at low forces. The trapped bead is moved in the direction perpendicular to the microscope cover slide so that cells can be indented in a similar fashion as with AFM. It was constructed to perform force spectroscopy experiments on cells at very low forces. 


\section{Introduction}

\subsection{Atomic Force Microscopy}

Since its invention in the 1980s by G. Binning et. al [1] AFM has developed extensively and opened new possibilities to explore sample characteristics on the nano meter scale. Beside its traditional utilization in material sciences, AFM entered chemical and biological sciences. In the biophysical field it is successfully used to gain insight into cell mechanics [2], viral capsids [3], and conformation changes of macromolecules [4]. Compared to other microscopy techniques, AFM allows to operate between the scales of diffraction limited light microscopy approaches and Electron microscopy (EM). Noticeably, although EM delivers even higher resolutions than AFM, it requires the fixation of the sample by coating or freezing to establish the vacuum necessary to work with electron optics. In contrast, AFM experiments can be performed under physiological conditions. Hereby, living cells and dynamics of enzymes can be studied. Nevertheless, to scan a sample in liquid with AFM the immobilization of the sample on the substrate is still required. The AFM working principle relies on image reconstruction from mechanical information, analogue to a person acquiring an internal representation of an object just by its haptic percept. The main components building up an AFM are shown in fig. 2.1. The mechanical contact with the sample is established by a cantilever which is basically a beam with a sharp tip at its end. The radii of the tip apex are very small and typically lie in the range from 2 to $30 \mathrm{~nm}$ for current cantilevers. To get the tip in contact with the sample, piezo actuators move the cantilever with respect to the sample. Contact is identified by the bending of the cantilever when it touches the sample. The detection of cantilever bending is realized by a laser beam which is focused on the backside of the cantilever and reflected on a photo detector, placed several centimeters away. Mostly, a quadrant photo diode (QPD) 
is used which allows the detection of vertical and horizontal changes of the laser spot.

The bending of the cantilever causes a change in the deflection angle of the laser beam which translates to a vertical shift of the laser spot on the QPD. The optical arrangement of cantilever and detector acts via the deflected laser as an amplifier for cantilever displacement. Noteworthy, the cantilevers can be very soft so that they already bend when the tip apex surface electrostatically interacts with the sample surface.

During imaging, the contact force, re-

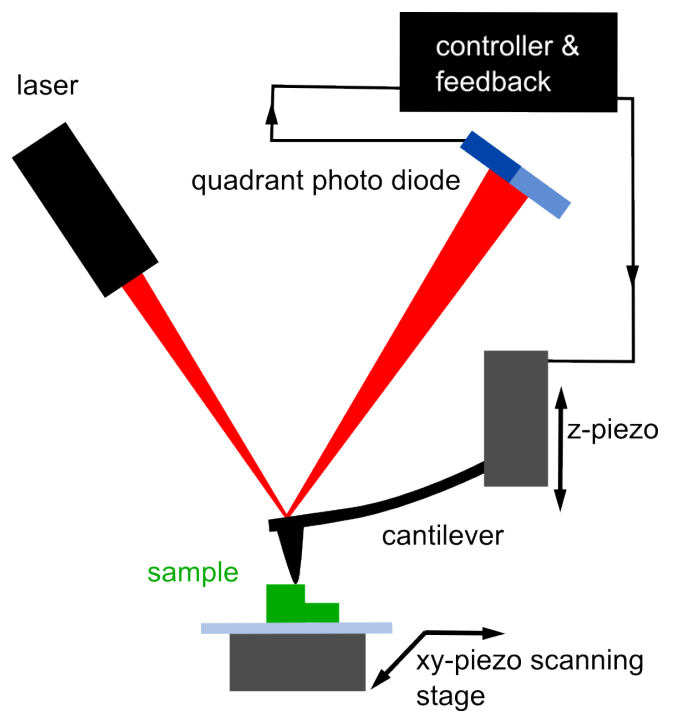

Figure 2.1: AFM principle with its instrumental core components. spectively the force acting on the sample, should be as small as possible to prevent sample deformations which would distort the real contour of the object. This is performed by the AFM controller. The controller keeps the pre-set contact force constant by correcting the z-piezo position while the tip is scanning over an object in the xy-plane. The image is reconstructed from the substrate height at discrete xy-positions. The substrate height is deduced from the way the z-piezo has to travel to maintain the bending and, thus, the contact force constant. AFM can be performed in different operation modes. The explanations above refer to the so called contact mode.

Imaging biological samples without distortions requires a low force exerted by the cantilever to the sample. The contact mode imaging mentioned above is rarely used for biological samples because of the large lateral forces that are exerted by the tip on the sample. Instead, mostly tapping mode is applied for imaging. In tapping mode the cantilever is oscillated with a defined drive amplitude and frequency. When the cantilever is brought close to the surface, the amplitude will decrease due to the tip-sample interactions. Because the tip is touching the sample for only a fraction of the oscillation cycle, the lateral forces are much smaller than in contact mode. Mostly, the cantilever is excited at its resonance frequency so that the drive signal can be kept small. In the tapping imaging mode the controller 
compares the set amplitude with the actual amplitude and the z-piezo position is continuously corrected to maintain a constant amplitude during scanning.

\subsubsection{Cantilever calibration}

For the determination of the forces in contact mode imaging and during deformation experiments with AFM, the force vs. bending relation of the cantilever must be known. The bending corresponds to the so called deflection $z$ which measures the displacement of the cantilever end from its equilibrium position. For a linear spring the relation between force $f$ and extension is given by Hooke's law. According to this relation, the cantilever bending can be converted into a force: $f=\kappa z$ when the spring constant $k$ of the cantilever is known. The approximate spring constant of cantilevers is given by the manufacturer but should be calibrated for every cantilever individually. The Euler-Bernoulli beam theory can be applied to the cantilever to calculate the spring constant from its dimensions and material properties:

$$
k=\frac{E w t^{3}}{4 L^{3}} \quad \nu_{r}=\frac{1}{2 \pi} \sqrt{\frac{\kappa}{m}}
$$

where $E$ is the Young's modulus of the cantilever material, $w$ is the width, $t$ the thickness, and $L$ the length of the beam. Another important cantilever parameter is the kinetic resonance frequency $\nu_{r}$ which also depends on its mass $m$. These calculations could be straightforward but since the exact dimensions are difficult to obtain, the results are not always accurate. Moreover, the situation becomes more complex when taking coated cantilevers into account. Metal coatings (mainly noble metals) are often applied to the backside of the cantilever to increase the reflectance of the detection laser beam.

In practice, calibration is mainly done by thermal noise methods although there are several other methods available [5]. The thermal method is based on the equipartition theorem [6]. In a thermodynamic system each degree of freedom is assigned to the same thermal energy so that a freely fluctuating cantilever eigenmode will 
be excited by the bombardment of the surrounding air or water molecules with the energy:

$$
\frac{1}{2} k_{b} T=\frac{1}{2} \kappa\left\langle z^{2}\right\rangle
$$

where $k_{b}$ is the Boltzmann constant, $T$ the absolute temperature and $\left\langle z^{2}\right\rangle$ the mean square amplitude measured over a statistically sufficient time interval. If the magnitude is known one can derive $\kappa$. Because external noise sources like mechanical vibrations can easily lead to an increase of $\left\langle z^{2}\right\rangle$ this method is not so robust.

For a more accurate calibration the cantilever fluctuation distribution is recorded as the Power Spectral Density (PSD). It represents the fluctuations at the frequency domain. The PSD includes the cantilever fluctuations and eventually noise sources with their characteristic frequency. After mounting the cantilever in its holder in the sample chamber, it is equilibrated in the surrounding buffer medium. Then the fluctuations are picked up by the photo-detector and the induced voltage is recorded as time series $V(t)$. The time series is then Fourier transformed and the $\mathrm{PSD}_{v}$ is obtained in the units $\left[V^{2} / H z\right]$ [7]. Translation to the cantilevers $\mathrm{PSD}_{m}$ in $\left[m^{2} / H z\right]$ is done by:

$$
P S D_{m}=P S D_{v} \cdot \frac{1}{S^{2}} \cdot \frac{1}{\cos ^{2} \alpha} \cdot \chi^{2}
$$

Here $S$ resembles the sensitivity of the cantilever bending in $[\mathrm{nm} / \mathrm{V}]$ with respect to the QPD voltage which is obtained by performing a force distance curve on a solid substrate. Because the z-piezo is calibrated in $\mathrm{nm}$, the force distance curve gives the relation between bending and voltage directly. The cantilever is usually mounted at an angle $(\alpha)$ in the range of $5^{\circ}-15^{\circ} \cdot \chi^{2}$ is a factor comprising the position and size of the laser spot on the backside of the cantilever. It can be assumed as a constant of 1.09 [8]. A typical power spectrum is shown in fig. 2.2. 


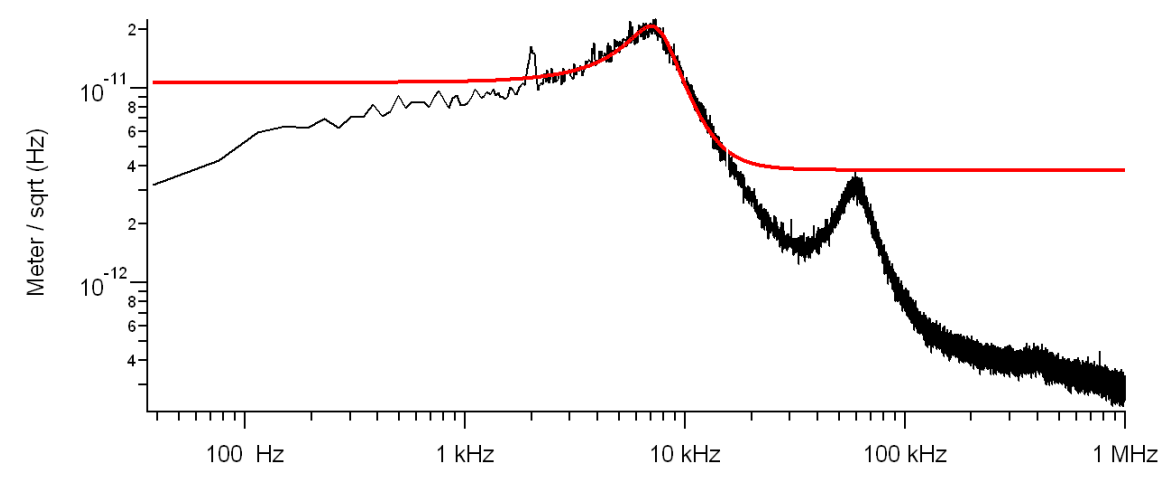

Figure 2.2: Fitting the cantilever model to the cantilevers PSD delivers its spring constant. Black: thermal noise PSD of a Olympus BL-RC150VB-HW cantilever. Red: fit of the cantilever fluctuation model (equation 2.4), to derive $\kappa$. The fit includes only the first harmonic.

To derive $\kappa$ from the PSD, the cantilever fluctuations around the resonance frequency are described as a harmonic oscillator and fitted to the recorded PSD. In this approach the mean square amplitude is normalized to $k_{B} T / 2$, as required by the equipartition theorem. The final function to be fitted to the $P S D_{m}$ is given by [5]:

$$
\left\langle z^{2}(\nu)\right\rangle=\frac{A}{\nu}+B+\frac{\left\langle z^{2}\left(\nu_{r}\right)\right\rangle}{Q^{2}} \cdot \frac{1}{\left[1-\left(\frac{\nu}{\nu_{r}}\right)^{2}\right]^{2}+\left[\frac{\nu}{\nu_{r} Q}\right]^{2}}
$$

Here $\nu$ is the frequency. The free parameters of function 2.4 are: $A, B, Q,\left\langle z^{2}\left(\nu_{r}\right)\right\rangle$, $\nu_{r} . A$ and $B$ describe the pink noise (frequency dependent) and white noise (frequency independent). $Q$ is the damping related quality factor. $\left\langle z^{2}\left(\nu_{r}\right)\right\rangle$ is the mean square amplitude (at the resonance frequency of the first eigenmode $\nu_{r}$ ), which is equal to:

$$
\left\langle z^{2}\left(\nu_{r}\right)\right\rangle=\frac{k_{B} Q T \Delta \nu}{\pi \kappa}
$$

Since all magnitudes in equation 2.5 are known or can be derived from the fit (red in fig. 2.2) of equation 2.4, the value of $\kappa$ can now be calculated. 


\subsection{TIRFM}

TIRFM is a technique to excite fluorescent molecules in proximity to a surface. It was pioneered by J.E. Ambrose [9] and further developed by D. Axelrod [10]. Due to its exceptional signal to noise ratio, it became an important tool to study various surface associated biological processes like cell adhesion, binding of neurotransmitters, or deposition of plasma membrane proteins. The background fluorescence of sample regions of no experimental interest located further away from the surface is reduced by a spatial confinement of the electromagnetic excitation field. The physical confinement of the excitation field is realized by the creation of an evanescent wave which penetrates the sample by less than $\approx 200 \mathrm{~nm}$. The creation of the evanescent wave is a main feature of this microscopy method and requires a Total Internal Reflection (TIR) of a wave front.

In TIRFM, TIR is realized in two main ways: In the traditional approach, prisms, glass cubes, or wave guides attached to the microscope cover slip are used to create TIR by adjusting incidence angles and refractive indices. Modern approaches employ a high Numerical Aperture (NA) microscope objective to create the TIR. The latter approach is also the method of choice for TIRFM used in a combination with AFM. Since the half space above the objective must enable access to the sample by the AFM, it

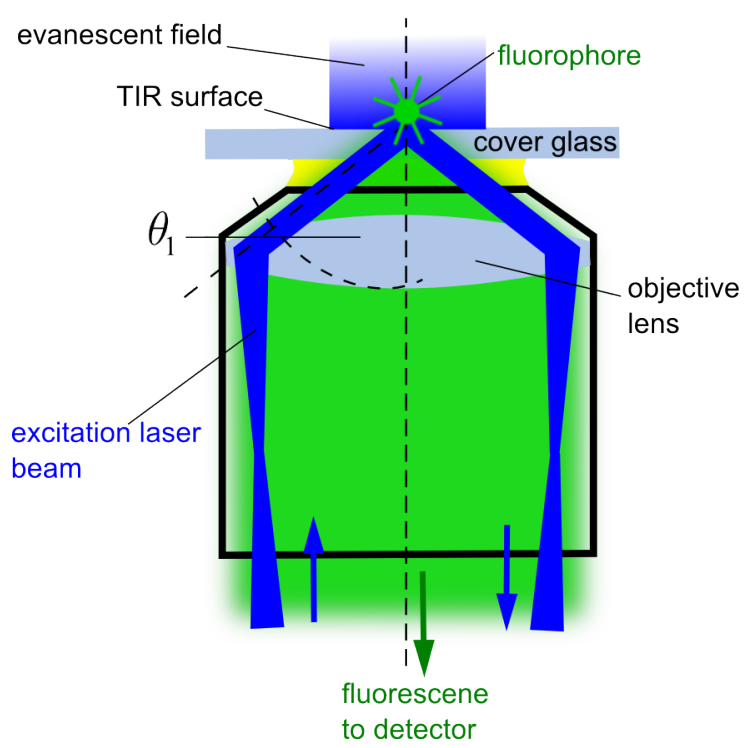

Figure 2.3: Objective type TIRFM. cannot be occluded by prisms or wave guides. Noteably, most AFMs can only be operated in an upright configuration. Therefore, the light microscope part of the setup must be configurated invertedly. The detailed arrangement of the instrument combination is explained in section 4.1.1. In objective type TIRFM, fluorescence excitation and detection are realized both through the objective (2.3). To achieve TIR through an objective its NA, given by $N A=n \sin \alpha$, must allow light incidence angles $\left(\theta_{1}\right)$ greater than the crit- 
ical angle (as explained below). $n$ is the index of refraction and $\alpha$ is the half-angle of the maximum cone of light that can enter or exit the lens. The following section describes the evanescent field from a wave optical perspective.

\subsubsection{TIR}

A light beam is thought of as a plane wave with its wave vector $k$ being parallel to its propagation direction. It travels through a transparent medium with a high refractive index $n_{1}$ and encounters an interface followed by a medium with a lower refractive index $n_{2}$ (fig.2.4). Depending on its incidence angle $\theta_{1}$, it undergoes refraction, reflection, and total internal reflection at high angles.

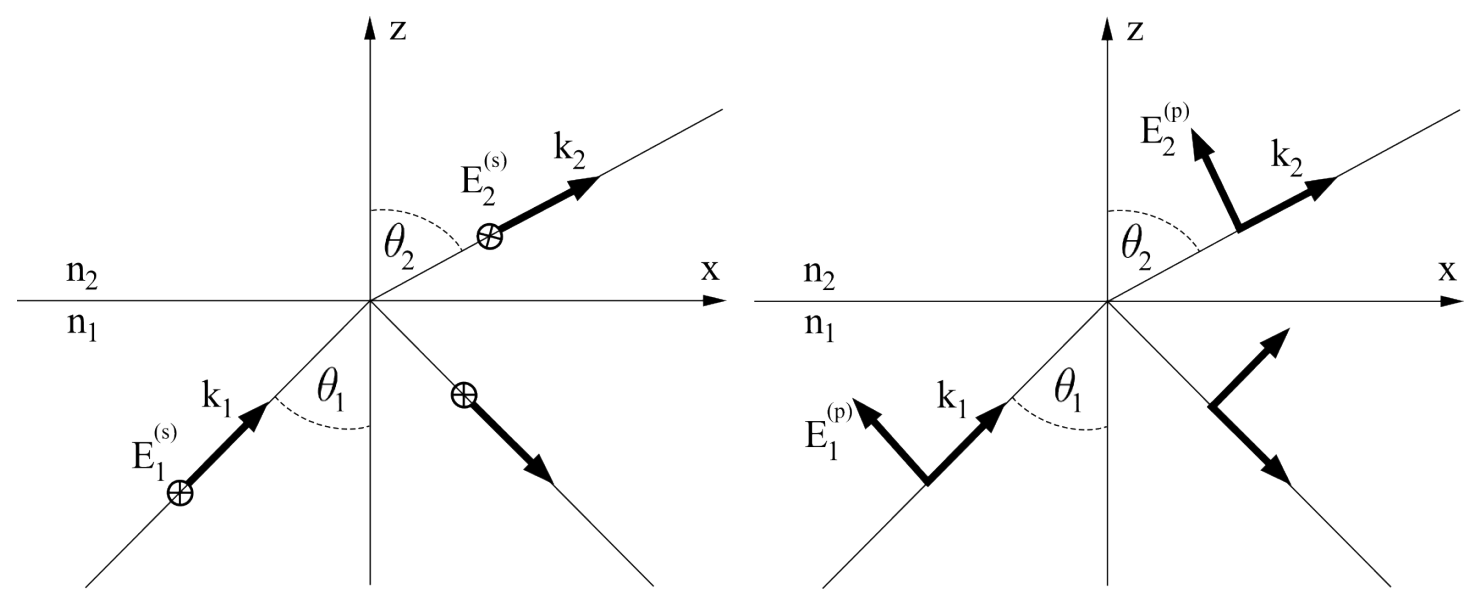

Figure 2.4: Refraction and reflection of a $s$-polarized and $p$-polarized plane wave at an interface. $n_{1}>n_{2}$

The material properties are discontinuous at the interfaces. Since Maxwell's equations must also hold for this situation, there are boundary conditions requiring the transverse components of the wave vector $\left(k_{x}, k_{y}\right)$ to be conserved [11]. While the $y$ direction stands orthogonal to the paper plane and parallel to the $s$-polarised $E$ field, the $p$-polarization is located parallel to the incidence plane or paper plane (compare $\otimes$ in fig.2.4). The complex transmitted field vector $E_{2}$ can be expressed 
as [12]:

$$
E_{2}=\left[\begin{array}{c}
-E_{1}^{(p)} t^{p}\left(k_{x}\right) k_{2 z} / k_{2} \\
E_{1}^{(s)} t^{s}\left(k_{x}\right) \\
E_{1}^{(p)} t^{p}\left(k_{x}\right) k_{x} / k_{2}
\end{array}\right] e^{i k_{x} x+i k_{2 z} z}
$$

where $t^{p}, t^{s}$ are the Fresnel coefficients for the corresponding polarizations. Transforming $\quad k_{2 z}=\cos _{2} k_{2} \quad$ with $\cos ^{2} \theta+\sin ^{2} \theta=1 \quad$ delivers $k_{2 z}=k_{2} \sqrt{1-\sin ^{2} \theta_{2}} \quad$ with Snells refraction law $\sin \theta_{1} n_{1}=\sin \theta_{2} n_{2} \quad$ :

$$
k_{2 z}=k_{2} \sqrt{1-\frac{n_{1}^{2}}{n_{2}^{2}} \sin ^{2} \theta_{1}}
$$

Since $n_{2}<n_{1}$, the expression under the square root turns first into: $\quad\left(1-\frac{n_{1}^{2}}{n_{2}^{2}} \sin ^{2} \theta_{1}\right)=$ 0 with $\theta_{1}=\theta_{c}$ (the critical angle) and then imaginary for $\theta_{1}>\theta_{c}$. Expressing equation 2.6 as a function of $\theta_{1}$ leads to:

$$
E_{2}=\left[\begin{array}{c}
-i E_{1}^{(p)} t^{p}\left(\theta_{1}\right) \sqrt{\frac{n_{1}^{2}}{n_{2}^{2}}} \sin ^{2} \theta_{1}-1 \\
E_{1}^{(s)} t^{s}\left(\theta_{1}\right) \\
E_{1}^{(p)} t^{p}\left(\theta_{1}\right) \frac{n_{1}}{n_{2}} \sin \theta_{1}
\end{array}\right] e^{i \sin \theta_{1} k_{1} x-\gamma z}
$$

where $\gamma$ describes the decay of the field in $\mathrm{z}$ direction.

$$
\gamma=k_{2} \sqrt{\frac{n_{1}^{2}}{n_{2}^{2}} \sin ^{2} \theta_{1}-1}
$$

Equation 2.8 describes a field that propagates along the surface but decays exponentially into the medium of transmittance. This configuration is named evanescent wave. From fig. 2.5 it becomes clear that the evanescent wave can be practically confined to sub-wavelength. Its length is not diffraction limited. For the 
s-polarized incident light the evanescent electric field vector is perpendicularly polarized to the plane of incidence. For the p-polarized incident light a non-zero longitudinal component and a phase lag exist which causes the evanescent field vector to be ellipticly polarized when observed along the $x$ direction.

Using $|\mathbf{k}|=\left|\frac{2 \pi}{\lambda}\right|$ and $\lambda_{2}=\frac{\lambda_{1}}{n_{2}}$ delivers:

$$
\gamma=\frac{2 \pi}{\lambda_{1}} \sqrt{n_{1}^{2} \sin ^{2} \theta_{2}-n_{2}^{2}}
$$

$I(z)=I(0) e^{-\gamma z}$ describes the intensity decay $I(z)$ of the evanescent wave. Fig. 2.5 shows the decays for different incidence angles (see also the measured decays (fig.4.9) in section 4.1.5).

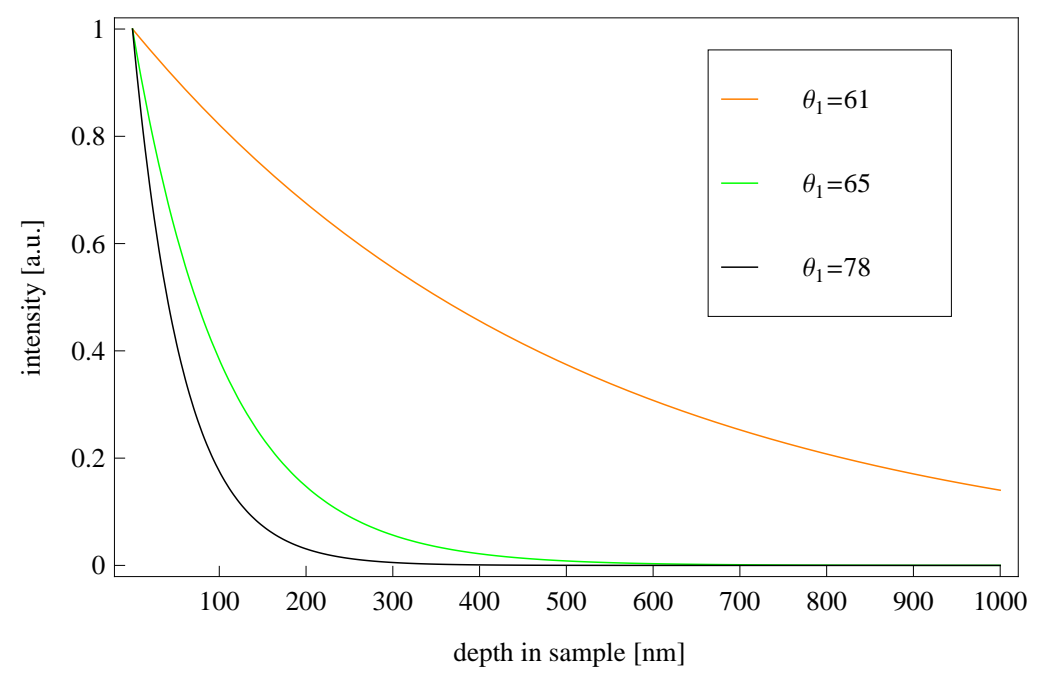

Figure 2.5: Intensity decay of an evanescent wave. The angle range is limited by the used objective with an NA of 1.49 from $61^{\circ}-78^{\circ}$. The parameters $n_{1}=$ $1.53, n_{2}=1.34, \lambda=488 \mathrm{~nm}$ are adapted to our experimental conditions.

The penetration depth $d$, is defined as the distance where $\gamma z=1$. This is the point where $I(z)=\frac{I(0)}{e}$. The intensity is proportional to the squared amount of the electric field amplitude. Therefore, we get:

$$
d=z=\frac{1}{\gamma}=\frac{\lambda_{1}}{4 \pi}\left(n_{1}^{2} \sin ^{2} \theta_{2}-n_{2}^{2}\right)^{\left(-\frac{1}{2}\right)}
$$




\subsubsection{TIR excitation}

Since the term TIR is a distinct expression, it is conclusive that there is no net energy transport into the optical thinner medium. However a local probe like a single fluorophore can absorb energy from the electrical field [12]. The probability of absorption is proportional to $\left|\mu_{a} \mathbf{E}\right|^{2}$ where $\mu_{a}$ is the absorption dipol moment. For random oriented dipoles the absorption probability rate is proportional to the 'intensity' $I_{s, p} \equiv\left|\mathbf{E}_{s, p}\right|^{2}$. The evanescent wave intensities at $z=0$ are given by $I_{p}=I_{x}+I_{z}$ and $I_{s}[10]:$

$$
\begin{aligned}
& I_{x}(0)=\left|A_{p}\right|^{2} \frac{\left(4 \cos ^{2} \theta_{1}\right)\left(\sin ^{2} \theta_{1}-n^{2}\right)}{n^{4} \cos ^{2} \theta_{1}+\sin ^{2} \theta_{1}-n^{2}} \\
& I_{z}(0)=\left|A_{p}\right|^{2} \frac{4 \cos ^{2} \theta_{1} \sin ^{2} \theta_{1}}{n^{4} \cos ^{2} \theta_{1}+\sin ^{2} \theta_{1}-n^{2}} \\
& I_{p}(0)=\left|A_{p}\right|^{2} \frac{\left(4 \cos ^{2} \theta_{1}\right)\left(2 \sin ^{2} \theta_{1}-n^{2}\right)}{n^{4} \cos ^{2} \theta_{1}+\sin ^{2} \theta_{1}-n^{2}} \\
& I_{s}(0)=\left|A_{s}\right|^{2} \frac{4 \cos ^{2} \theta_{1}}{1-n^{2}}
\end{aligned}
$$

with $n=n_{1} / n_{2}$. Here, $A_{p, s}$ are the incident electric field amplitudes, before the interface. These equations describe the intensities for the range $\theta_{1}>\theta_{c}$ and are plotted in fig. 2.6. The intensities for the sub-critical range $\theta_{1}<\theta_{c}=61^{\circ}$ are based on refraction intensities calculations with Fresnel coefficients as in [13]. See also the measured angle dependencies (fig. $4.16 \& 4.17$ ) in section 4.1.5. 


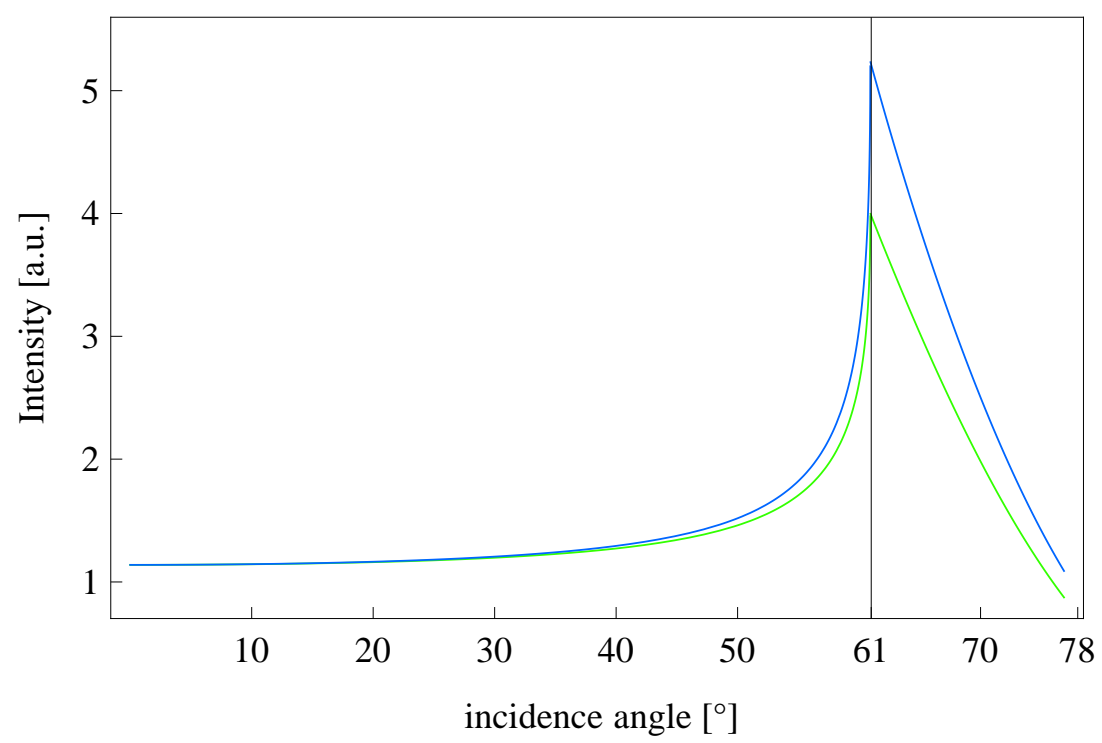

Figure 2.6: Intensity $I_{p, s}(0)$ plotted versus $\theta_{1}$. S-polarization in green and ppolarization in blue. The incident intensities $\left|A_{s, p}\right|^{2}$ are set equal. The parameters $n_{1}=1.53, n_{2}=1.34, \lambda=488 \mathrm{~nm}$ are adapted to our experimental conditions.

The intensity for supercritcal incidence angles are enhanced up to five times compared to the intensities at $\theta_{1}=0$. Physically, this can be explained by a surface polarization induced by the incoming plane wave [12].

Placing the AFM tip inside an electromagnetic field can lead to a local (near field) enhancement around the tip apex. This applies to SiliconNitrate (SiN) tips [14] on a moderate level and to metal coated tips to a larger extend [15]. For tip radii of $r_{\text {tip }}<\lambda$ the near field under the tip is confined to dimensions of $\Delta x \approx r_{\text {tip }}$ where $\Delta x$ lies orthogonally to the longitudinal tip axis [16]. The enhancement by metal tips strongly depends on the polarization of the electromagnetic field. A field owning a polarization component parallel to the tip surface is able to periodically displace free electrons of the metal along the direction of the oscillating electrical field. The surface charges can form oscillating standing waves resulting in surface plasmons resonance. This is commonly associated with strong field enhancements. Since the longitudinal tip dimensions are in the range of light wavelengths, an additional "antenna" occurs, which contributes to the local field enhancement at the tip apex [12]. Due to the conical tip shape and the electron displacement, the excitation field creates a huge accumulation of surface charges at the small tip apex and leaves less shielded ions above the tip apex. According to Gauss law, these charges give rise to a dipole field which increases the intensity to a level higher 
than the illumination intensity at the tip apex. The less strong enhancement of non-metal tips is due to so called 'field line crowding' [12].

In the section above, an optical field designated for fluorescence excitation and its enhancement by an AFM tip was introduced. Another effect of the tip can be observed when it is placed close to an excited fluorescent molecule. Here, especially metal coated tips can quench the fluorescence of the molecules. Quenching is commonly referred to as a reduction of the quantum efficiency which describes the relation between energy emission and absorption. This relation strongly depends on the distance between tip and fluorophore. As the tip enters the near field of the molecule, it changes the rate of radiative transitions and, hereby, the emission of light per time [17]. Surface Plasmon excited by the near field of the molecule on the metal tip surface are linked to decreased and increased radiative rates of the molecule [18]. Those depend on the spatial separation. However, at distances of less than $5 \mathrm{~nm}$ an entirely non-radiative short-ranged energy transfer becomes dominant. Evidentially, the near-field of the molecule penetrates the metal and excites electron-hole pairs that quench fluorescence [19].

\subsection{Optical Tweezers}

The effect of optical trapping was first postulated and demonstrated by Arthur Ashkin over 30 years ago. The underlying physical principles of his experiments were based on radiation pressure exerted by counter propagating laser beams. Nowadays, optical trapping has been further developed to the single beam gradient force-method [20]. Here, a tightly focused laser beam is used to exert an attractive force to a dielectric microsphere (bead). The trapping occurs due to a balance between optical radiation pressure and the direction of the intensity gradient in the focused field. The common way to focus a laser strong enough for a trap is to use a microscope objective with a high NA.

Since the technique also works in liquid environments, it is suitable for applications with bio-samples like living cells or single bio molecules. Different theoretical concepts exist regarding the forces which hold a bead in a focused laser beam. The concepts depend on the relation between the bead radius and the laser wavelength. In the following, the ray optics approach will be shortly introduced. 


\subsubsection{Trapping forces and the ray optics model}

To explain the forces in the ray optics regime, it is useful to divide them into two components: The first component can be abstracted as a scattering part in the direction of the beam propagation. The second, less perceptible component corresponds to photons being refracted on their way through the bead. Both parts occur due to the photons momentum $\vec{p}$ which is $\vec{p}=\hbar \vec{k}$. Here, $\vec{k}$ resembles the wave vector and $\hbar$ the reduced Planck constant. The trap is formed by a strongly focused laser beam with a Gaussian intensity profile. Imagine a single ray or photon which is refracted twice at the surface of the bead. It will enter with a certain momentum and exit with a different one (due to refraction) (fig.2.7). The net momentum change $\Delta p_{1}$ is calculated by the vectorial difference between both [12]. Momentum conservation requires the transfer of the momentum to the bead points in the opposite direction.
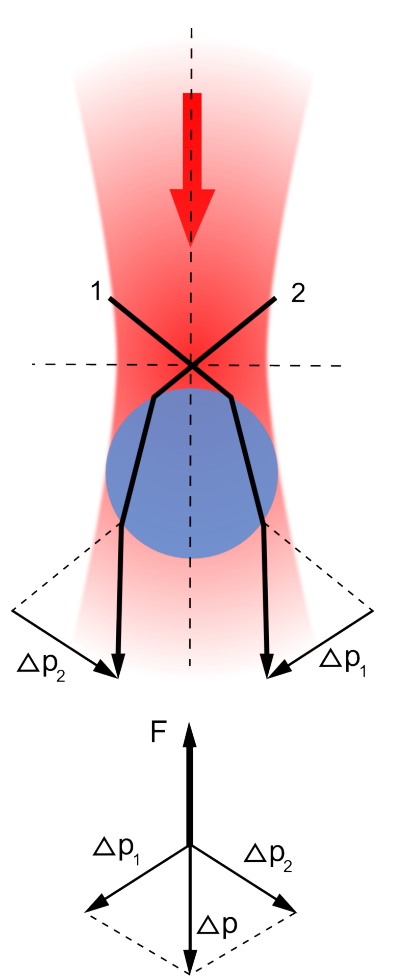

(a) equilibrium position
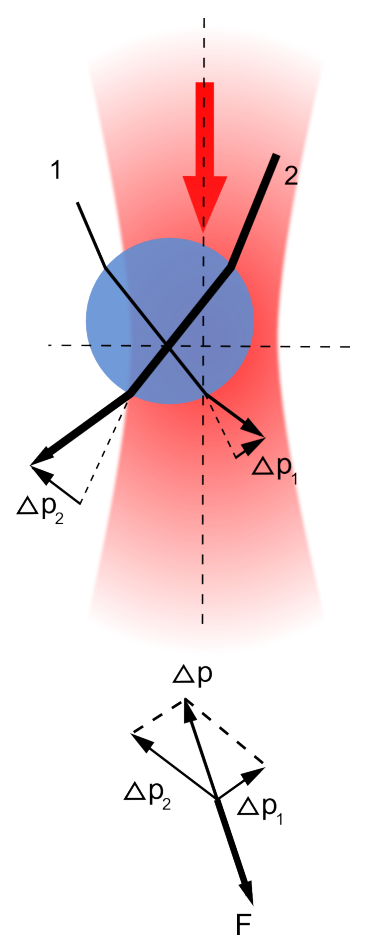

(b) bead displacement

Figure 2.7: Optical trapping principle. 2.7a Stable trapping position, the forces caused by scattered and refracted photons are in equilibrium. $2.7 \mathrm{~b}$ The bead is displaced from the trap center. The intensities of ray 1 and 2 are different. So, the amount of refracted photons per area and time are also unequal. The transferred momenta cause a restoring force in direction to the equilibrium position. 
The full trapping principle becomes clear if we consider the refraction of two rays, respectively two photons. In figure 2.7 a ray 1 has the same intensity as ray 2 (with the intensity representing a quantity of photons per time). The total momentum change $\Delta p$ is calculated by the vectorial sum of $\Delta p_{1}, \Delta p_{2}$. Therefore, the force acting on the bead $f$, tries to pull it to the focus center. In a static position, $f$ is in equilibrium with the scattering forces (not shown). In figure $2.7 \mathrm{~b}$ the bead is displaced from its equilibrium position. The intensity of ray 2 is higher since the bead center is displaced laterally from the intensity focus. Hence, the resulting momentum changes $\Delta p_{1}$ and $\Delta p_{2}$ are unequal. The total restoring force acting on the bead is pointing towards the equilibrium position. The total trapping force can be calculated by summing up the forces of the different rays assumed to represent the light beam [21].

\subsubsection{Trap calibration}

As if it was suspended by springs, the bead will be kept in the center of the focused laser beam by the trapping forces acting on it. If the bead is moved out of the trap center due to an external force in the experiment, the "springs" will try to pull it back to the equilibrium point. For small displacements out of the trap center the trapping potential is harmonic. Hooke's law $f=\kappa z$ is used to describe the displacement $(z)$ of the force probe. For measuring forces in such experiments, the knowledge of the spring constant $(\kappa)$ is required. To calibrate the spring constant, the measured fluctuations of a trap bead can be used. The diffusive Brownian motion experienced by a particle in a viscous liquid is described by the Langevin equation. A trapped bead experiences the optical restoring force and responses to the thermal (Brownian) force. The Langevin equation is then given by ${ }^{\mathrm{i}}$ [22]:

$$
F(t)=\gamma \frac{d z}{d t}+\kappa z
$$

The drag coefficient of the bead is given by Stokes drag equation $\gamma=6 \pi \eta r$, where $r$ is the bead radius and $\eta$ the dynamic viscosity of the solvent. $F(t)$ represents

\footnotetext{
${ }^{\mathrm{i}}$ Since for micrometer sized objects the Reynolds number is very low $\left(\sim 10^{-4}\right)$, inertial forces
} are neglected. 
the random thermal force. Its average value is 0 . The PSD $\left(S_{F}(\nu)\right)$ of $F(t)$ is also constant (white noise). For the described mechanical systems, the Nyquist formula expresses the thermal white noise magnitude in terms of the drag coefficient [23]:

$$
S_{F}(\nu)=\left|\mathscr{F}^{2}(\nu)\right|=4 \gamma k_{b} T
$$

Here $\mathscr{F}(\nu)$ is the Fourier transform of $F(t)$. Fourier transforming equation 2.16, with $\varsigma(\nu)$ as the Fourier transform of $z(t)$ and taking the modulus on both sides, delivers the PSD of the position signal [23].

$$
S_{z}(\nu)=\left|\varsigma^{2}(\nu)\right|=\frac{k_{b} T}{\gamma \pi^{2}\left(\nu_{c}^{2}+\nu^{2}\right)}
$$

Here $\nu_{c}$, the characteristic or corner frequency, is defined by: $\nu_{c}=\kappa / 2 \pi \gamma$. Fitting equation 2.18 to the recorded bead fluctuations (fig. 2.8) with $\nu_{c}$ as a fit parameter allows to calculate $\kappa$.

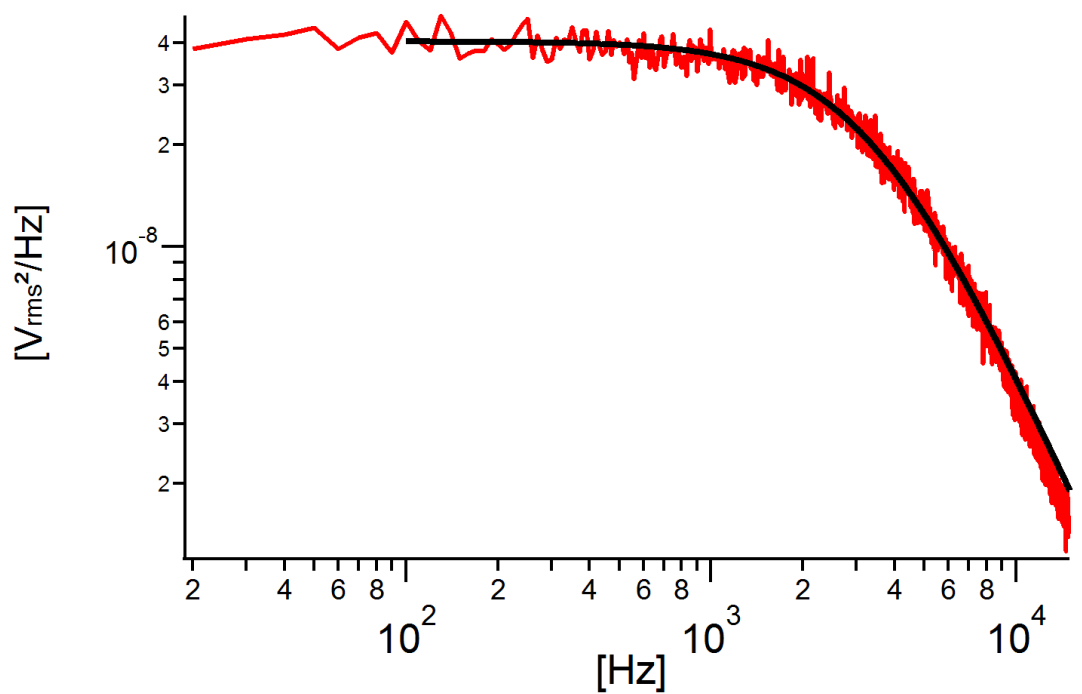

Figure 2.8: PSD (red) of a trapped bead $(\varnothing=760 \mathrm{~nm}) 10 \mu \mathrm{m}$ inside the sample chamber. In black the fit of equation 2.18 is shown, which delivers $\nu_{c}$ and allows to calculate $\kappa$. PSD is acquired on the instrument described in section 4.4.

For frequencies $\nu \gg \nu_{c}$, the PSD decreases as $1 / \nu^{2}$, showing that at short timescales the trapping forces do not hinder the Brownian motion. At frequencies $\nu \ll \nu_{c}$, 
the PSD is roughly constant: $S_{z}(\nu)=S_{0}=4 \gamma k_{b} T / \kappa^{2}$. This approximation opens the possibility to derive $\kappa$ without knowing the bead dimensions $(\gamma)$. To do so, $\gamma$ can be eliminated from the latest expression by using its connection to $\nu_{c}$. So finally, we have two ways to determine $\kappa$ given by the equations:

$$
\kappa=\frac{2 k_{B} T}{\pi S_{0} \nu_{c}} \quad \text { or } \quad \kappa=2 \pi \gamma \nu_{c}
$$

To calculate forces we need to know not only $\kappa$ but also the bead displacement from the trap center $z$. The detector delivers a voltage that depends on the position, so that we have to know the relation between displacement and voltage. Therefore, the linear sensitivity $p$ must be calibrated. The displacement PSD is given by $S_{z}(\nu)=S_{v}(\nu) \cdot p^{2}[24]$. Here, $S_{v}(\nu)$ is the detected voltage PSD, which can be described by $S_{v}(\nu)=\left(S_{0}^{V} \nu_{c}^{2} /\left(\nu_{c}^{2}+\nu^{2}\right)\right)$. The quantity $S_{0}^{V}$ is the low frequency limit in $\left[V^{2} s\right]$. The response PSD (eq. 2.18) is also known since $\nu_{c}$ can be derived from a fit to the voltage PSD and $\gamma$ can be calculated from the bead dimensions. So, the calibration factor $p$ is the only unknown factor and, hence, determined by these relations. For $p$ in $[m / v]$ we get:

$$
p\left[\frac{m}{V}\right]=\left[\frac{k_{b} T}{\pi^{2} \gamma S_{0}^{V} \nu_{c}^{2}}\right]^{\frac{1}{2}}
$$

This method for calibration determination agrees with other more direct methods within $\sim 20 \%$ [24]. Some experiments require an immobilization of the sample on a surface (cover glass). Therefore, the probe has to be brought quite close to the surface. There, a micro sphere would experience an increase in its dynamic drag coefficient. The drag increases as the displaced liquid molecules collide with the surface boundary. To perform the aforementioned calibration requires to incorporate the changed drag coefficient. A correction for the drag coefficient of a sphere moving normal to the surface $\gamma_{\perp}$ is given by [25]:

$$
\gamma_{\perp}=\frac{\gamma}{1-\frac{9 r}{8 h}+\frac{r^{3}}{2 h^{3}}-\frac{57 r^{4}}{100 h^{4}}+\frac{r^{5}}{5 h^{5}}+\frac{7 r^{11}}{200 h^{11}}-\frac{r^{12}}{25 h^{12}}}
$$


The displacement detection of the trapped particle in the presented optical tweezers instrument is realized by a QPD placed in the back-focal-plane (BFP) of the microscope condensor (see fig. 3.5 in section 4.4.1). A bead displacement changes the induced voltage on the four quadrons of the photo detector since it changes the far field interference pattern created by the bead presence [24]. The interference pattern depends on the trapping light scattered by the bead and the non-scattered trapping light. Both are influenced by the lateral position of the bead in the focus. For displacements in z-direction parallel to the optical axis an additional effect has to be taken into account: the Gouy phase shift. This phase shift occurs when a Gaussian beam passes through a tight focus [26]. While the non-scattered light experiences a Gouy phase shift, the scattered light preserves its native phase. The amount of light with and without a phase shift depends on the z-position of the bead in the focus. Hence, the complete spatial position is encoded in the light pattern detected by the QPD [27] .

\subsection{Biological samples}

\subsubsection{Cell mechanics and Parkinson's Disease associated LRRK2 mutations}

Together with P. Guerreiro of the Department for NeuroDegeneration and Restaurative Research of the Georg-August-Universität, Göttingen (Prof. T. Outeiro) I examined the effects of LRRK2 mutations on the mechanical properties in a $\mathrm{H} 4$ cell line. Mutations in LRRK2 contribute to the pathogenesis of Parkinson's Disease (PD). They are known to be the most frequent cause of inherited forms of the disease but they are also a risk factor for sporadic cases [28]. A big challenge in the LRRK2 research field is to better understand the functioning and dysfunctioning of the gene in the disease context. In order to achieve this goal, the identification of LRRK2-interacting proteins is critical to uncover cellular pathways and mechanisms. Gene ontology is a tool to describe how gene products behave in a cellular context. The list of interactors found for LRRK2 by gene ontology [29] indicates multiple interaction categories. Interactions with cytosolic structures like the cytoskeleton are found as well as connections to biological processes. Moreover, molecular functions are connected to LRRK2, too. This is supported by the 
finding that LRRK2 affects actin and microtubule dynamics [30]. Furthermore, experiments showed [31] the phosphorylation of Ezrin/Radixin/Moesin proteins by LRRK2 to promote the rearrangement of the actin cytoskeleton in neuronal morphogenesis. The influence on molecular functions is demonstrated by Meixner et al. [32] who found myosins as LRRK2 interacting partners. It was also demonstrated that LRRK2 promotes the abnormal aggregation and somatic accumulation of $\alpha$-synuclein. $\alpha$-synuclein is a transportation protein which is associated with Parkinson and other neurodegenerative diseases. Depending on the interplay of LRRK2 and $\alpha$-synuclein, different effects on the cytoskeleton can be expected [33]. Concluding, LRRK2 affects important building blocks of the cytoskeleton as well as crosslinkers between the plasma membrane and the cytoskeleton. The cytoskeleton which is made of several polymeric networks including actin, microtubules, and intermediate filaments, largely determines the structural and mechanical properties of the cell [34]. Thus, the mechanical properties of the cell can be an indicator of its cellular organization. This correlation is demonstrated by cancer cells which can be characterized by their changed mechanical behavior [35]. Therefore, AFM is a useful tool to to examine the condition of cells via its mechanical (viscoelastic) properties. In the past AFM has been used e.g. to characterize viscoelastic properties of individual glial cells and neurons in the Central nervous system (CNS) [36] and to measure astrocyte stiffness [37].

The expression of Green fluorescent protein (GFP) labeled LRRK2 in the H4 cell line shows distributions with characteristic patterns. TIRFM allows to identify those patterns and AFM enables mechanical measurements by indentation experiments. So, a combined experimental approach provides a way to reveal mechanical effects correlated with the LRRK2 distribution.

\subsubsection{Adenovirus infection pathway and structure}

We used AFM to disrupt single Adenovirus capsids and TIRFM to observe the release of the viral genome. The human Adenovirus (Ad) causes mainly respiratory, ocular, and gastrointestinal infections. Moreover, engineered virus versions are used as delivery vehicles in approaches for cancer gene therapy [38]. 
The Ad is one of the largest and most complex non-enveloped double-stranded DNA viruses. The adenoviral capsid has an icosahedral shape with diameters of about $90 \mathrm{~nm}$ [40]. The icosahedron consists of 20 triangular faces which are composed of hexon trimers (capsomers). In total, the virus has 240 of these capsomers (fig. 2.9). The icosahedron has also 12 vertices which are build as pentamers (pentons). From the pentons fibers protrude (not shown).

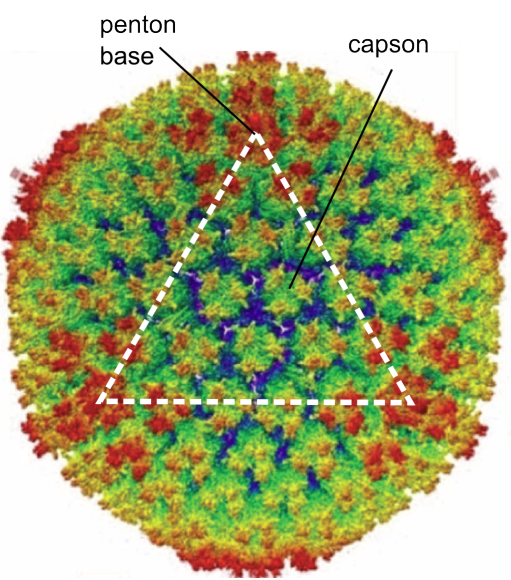

Figure 2.9: Overall structure of the Adenovirus. Adopted During the viral infection pathway of eukaryotic from [39]. cells, the viral shell must be disassembled to release the viral genome into the cell. Once the Adenovirus fibers bind to a cellular receptor, a process is induced by which a clathrin coated pit is formed taking up the particle into the cell [41]. The modification of the viral capsid starts at the plasma membrane were the protruding fibers are lost [42]. The formation of the clathrin pit marks the beginning of endocytosis. Thereupon, the viral particle is taken up by an endocytic vesicle. In this endosome the virus encounters an acidic $\mathrm{pH}$ which induces the loss of pentons and peripheral core proteins [43]. The low endosomal $\mathrm{pH}$ and the presence of Ad particles in combination with other factors (e.g. integrin) are believed to be responsible for endosome disruption [44]. After release, the partly disrupted virions travel to the nucleus mediated by microtubules and the minus-end directed motor complex dynein/dynactin. At the nuclear pore complex the capsid binds to a nuclear pore complex filament protein. The capsid is also indirectly linked to Kinesin-1 (running to the positive end of the microtuble). The Kinesin-1 motor protein disrupts the viral capsid and compromises the nuclear pore complex integrity. Finally the genome enters the nucleus [45].

To develop its full infective potential, the virus requires a maturation step during assembly. In this step the viral protease cleaves several capsid and core proteins. The cleavage leaves the proteins in a certain conformation so that a triggered change of their conformation can effect the virons structural integrity. Hence, the viral capsid is primed for the sequential disassembly procedure during infection 
[43]. An Ad2 mutant called TS1 lacks the maturation step. As a result, noncleaved protein precursors stabilize the connection between pentons and the viral core. Moreover, the virus core is more compact and stable due to condensing action of unprocessed proteins [43]. Therefore, the TS1 phenotype shows a disturbed structural disassembly and is not able to pass the endosome membrane during endocytosis [46], [47]. From these results it becomes clear that the maturation step is essential to render the Adenovirus to be fully infective.

The differences in structural disassembly between the WT and the TS1 mutant connects infectivity to mechanical stability. The dynamics of disassembly induced by mechanical fatigue were investigated for both virus types by Ortega-Esteban et al. [48]. The AFM based experiments revealed stability differences between WT Adenovirus and TS1. The mechanical disassembly was shown to start with a sequential loss of pentons at the vertices. This indicates their relevance as starting point for virus shell disintegration. The removal of pentons in the TS1 capsid required more AFM loading cycles (pushes/energy) than in the WT case. Moreover, the TS1 core remained more compact after shell disassembly as compared to the WT core. The height of the remaining core and shell structures were measured in AFM images for the WT group to be $\sim 35 \mathrm{~nm}$ while the TS1 height was with $\sim 70$ $n m$ much higher. The later value corresponds roughly to the core diameter plus an underlying layer of the shell.

Since the mentioned experiments and findings are based on AFM images, it is not possible to differentiate between genome and capsid structures after dissasembly. However, this could be realized by specific fluorescence labeling. Moreover, the images were acquired in tapping mode which requires hundreds of contacts between tip and sample. To investigate the spread of the remaining viron structures in a more diffusive driven way, an alternative to the ongoing AFM imaging is required. This leads to an experimental design which allows to induce a capsid rupture (mechanically) and to observe the spread of the genome (optically) afterwards. Here, the combined AFM-TIRFM instrument comes into play. In this setup the capsid dissasembly can be induced with a force distance curve (single push) and the genome spread can be followed by fluorescence labeling. The labeling can be done by using a DNA specific dye (YoYo-1) which increases its fluorescence $\sim 3000$ times when bound to the double-stranded DNA. The DNA binding can be observed in real time when the dye gains access after capsid breaking. 


\subsection{3 $70 \mathrm{~S}$ ribosome}

During protein synthesis the ribosome translates genetic information from messenger Ribonucleic acid (mRNA) into a polypeptide. The polypeptide is assembled from polymeric protein molecules (amino acids) whose sequence is controlled by the sequence of the mRNA. The decoding process is largely performed by transfer RNA (tRNA). The tRNA compares the mRNA information and delivers a specific amino acid to ribosome. After each added amino acid the mRNA strand has to move through the ribosome to allow the next tRNA to read the next information package (codon). For each amino acid added to the polypeptide the ribosome undergoes large conformational changes to facilitate translocation of the mRNA and tRNA and to recruit several translation factors. The SFB860 A4 ribosome project aims to get a deeper insight into the functioning of the ribosome. In a subproject AFM was used to measure small fluctuations in height by placing the AFM tip on top of a single ribosome, fig. 2.10. The preliminary results were interpretated in terms of contour changes of the ribosome which may occur during its enzymatic activity.

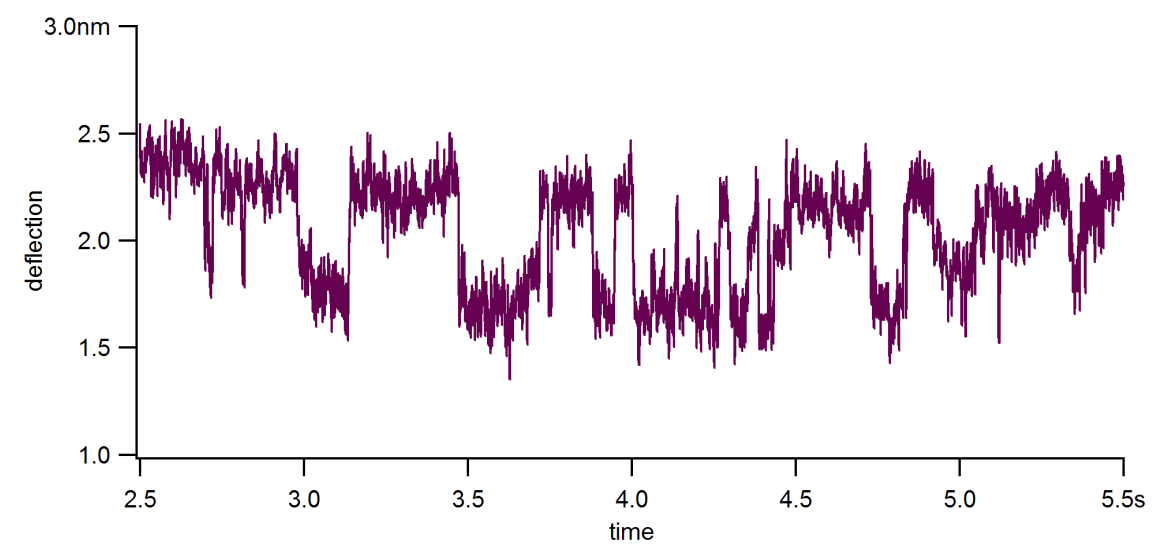

Figure 2.10: Fluctuations in height measured in preliminary AFM experiments on ribosomes. Data acquired by Frédéric Eghiaian.

The custom made AFM-TIRFM setup was also planned to complete the AFM observations with a method to visualize single factors (tRNAs and EFG) that are transiently bound to the $70 \mathrm{~S}$ ribosome during translation. This should be realized by simultaneous Total Internal Reflection Fluorescence (TIRF) microscopy detection of the fluorescently labeled factors. Combined experiments would then allow a correlation of the height fluctuations with the biochemical state of the ribosome. 
Therefore, the fluorescence of ribosome constructs labeled with a single dye molecule was characterized. The characterization includes bleaching times, signal to noise ratios, and possible detection frame rates. Moreover, in combined AFM-TIRFM experiments, the background light from AFM cantilevers during single molecule detection needs to be determined as well as the quenching potential of the cantilever tip.

\subsubsection{Actin-binding protein DBN-1}

In a cooperation with Eugenia Butkevich (Drittes Physikalisches Institut, GeorgAugust-Universität, Göttingen), the function of DBN-1, a C.elegans DreBriN-like family homolog was further investigated. DBN-1 is an actin-binding protein which is expressed in body wall muscle cells and is an essential component of the muscle contraction machinery. It effects the actin filament organization and stability in the sarcomeres (the basic unit of a muscle) which are critical for force generation by muscle cells. Interestingly, knockout of drebrin-like protein in mice leads to the development of hypertrophic cardiomyopathy [49]. Therefore, characterization of DBN-1 might help to further understand the pathogenesis of this disease on a molecular level.

In vivo, fluorescence observations of DBN-1 indicate the following relations (Butkevich et al. [50]): During muscle contraction DBN-1 alternates between the active myosin tail regions and the passive actin fiber regions in the sarcomere. Both regions represent the force generating structures. During force generation the myosin motor proteins attach to actin filaments and exert a power stroke that slide the myosin filament with respect to the actin filament. A contraction of the whole sarcomere structure results. In contracted sarcomeres DBN-1 is localized at the distal parts of actin filaments close to the actin anchoring base. Here, it regulates the relevant spacing of $\alpha$-actin and tropomyosin (another component of actin filaments). It is important to protect actin filaments from the interaction with $\mathrm{ADF} /$ cofilin since $\mathrm{ADF} /$ cofilin belong to a family of actin-binding proteins which disassembles actin filaments. A loss-of-function mutation in DBN-1 leads to a redistribution of $\alpha$-actin and tropomyosin along actin filaments. A partial depolymerization of F-actin upon muscle contraction results. In vivo, affected worms show an atactic winding movement. 
In vitro, binding of DBN-1 to actin filaments occurs with moderate affinity $(\mathrm{Kd}=$ $16.7+/-5.9 \mu M)$ at a stoichiometry of approximately 1 molecule of GST-DBN-1 to 16-17 molecules of actin mediated by the 3x coiled-coil domain (aa 154-368) [50]. In addition to actin binding, DBN-1 is also able to bundle actin filaments. The actin bundling activity of DBN-1 is most likely mediated by the presence of the three isolated coiled-coil domains neighboring the ADFH (actin-depolymerising factor homology) domain [50]. It is tempting to speculate that in vivo DBN-1 bundles neighboring actin filaments of the same sarcomere and/or filaments of the adjacent sarcomeres between dense bodies, thus strengthening them during contraction. Also in vitro DBN-1 bundles actin filaments. These bundles tend form loops which can be localized by TIRFM. Subsequent AFM imaging at single protein resolution helps to investigate the protein binding in more detail. 


\section{Methods and materials}

\subsection{TIRFM beam path design}

This section describes the optical layout to realize a TIRFM. It includes calculations, as well as the selection and arrangement of the necessary optical elements. These elements mainly collimate \& focus, combine \& separate, as well as translate $\&$ rotate the excitation and detection light beams in the setup. The mechanical layout and the combination with the AFM (MFP-3D, Asylum Research, USA) is described under section 4.1.

The implementation of objective type TIRFM requires a high NA which enables $\theta_{1}>\theta_{c}$, (compare fig. 3.1 and section 2.2.1). Normally, such high angles are only achieved with oil immersion objectives. For water based sample buffers $\theta_{c}$ can be reached with a $\mathrm{NA} \geq 1.33$. For practical operation, the NA must be substantially higher, starting at $\sim$ 1.4. Furthermore the NA limits the maximal achievable angle and, hence, the minimal achievable penetration depth of the evanescent field. In the presented setup an oil immersion objective with a NA of 1.49 was used (CFI Apochromat TIRF

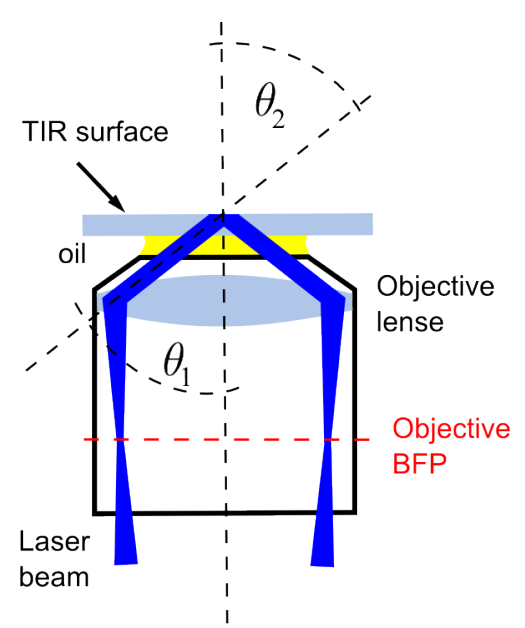

Figure 3.1: Principle of objective type TIR, compare section 2.2.1. $100 \mathrm{x}$, Nikon, Japan). The accessible TIR angles reach from $\theta_{c}=61^{\circ}$ up to $\theta_{1}=78^{\circ}$. This corresponds to a evanescent field penetration depth from $\approx 250 \mathrm{~nm}$ to $\approx 60 \mathrm{~nm}$, calculated with equation 2.11 in section 2.2.1. For focusing, the objective is mounted on a closed-loop controlled z-piezo stage (P-725.2CA + E-665.CR, Physik Instrumente, Germany). 


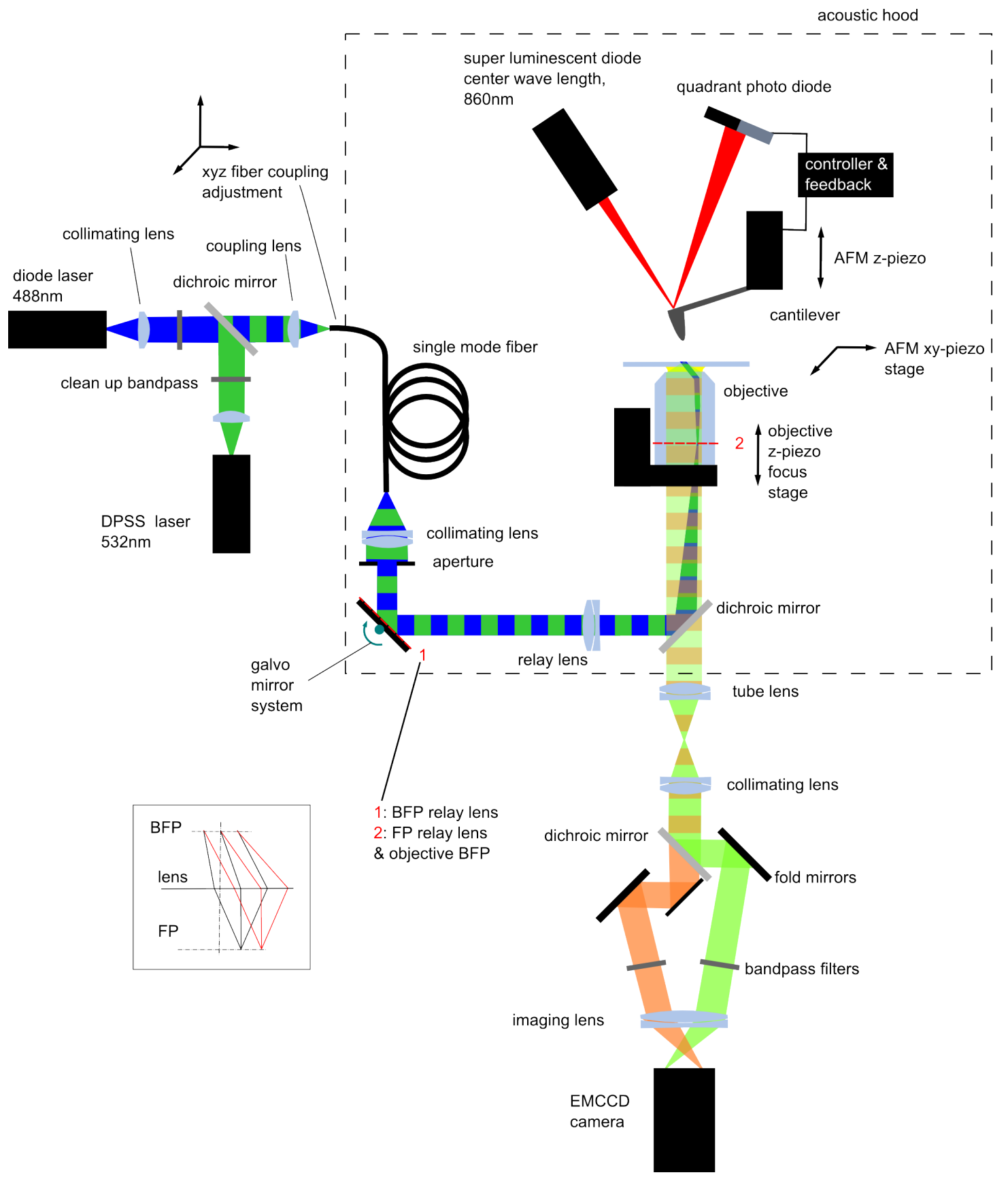

Figure 3.2: Functional layout of the combined AFM-TIRFM setup. The layout can be divided into 3 parts: The first (central) part located inside the acoustic damping hood contains the imaging parts of the both microscopes (Piezo stages, Objective, AFM head). The second part is an optical breadboard holding the excitation lasers, collimation and fiber coupling optics, and their adjustment stages. To avoid mechanical noise coupling, no rigid mechanical connection (besides the optical fiber) with the central structure exists (fig.4.2). The third part is the detection part with the tube lens, the image splitter, and the camera. It is mounted to the outside of the acoustic hood. So, again, there is no rigid connection to the core structure (only a connection to the base of the isolation table). Compare also: fig. 4.2. 
The TIRFM setup has two lasers for excitation (fig.3.2): The blue Laser Diode (LD) has a center wavelength of $\lambda=488 \mathrm{~nm}$ and a maximal optical output power of $P_{o}=80 \mathrm{~mW},($ NDS4116, Nichia, Japan). The green Diode Pumped Solid State Laser (DPSSL) owns a center wavelength of $\lambda=532 \mathrm{~nm}$ and a maximal optical output power of $P_{o}=40 \mathrm{~mW}$, (DJ532-40, Thorlabs, USA). Both laser spectra are narrowed down by clean-up filters. The LD as well as the DPSSL are driven in constant power mode. They are mounted on a thermal electric cooler with feedback loop to maintain a constant temperature inside their cavities. Both measures ensure a constant excitation intensity in the objective focus. The excitation intensity can be controlled and triggered (synchronized with the camera) via a custom written LabView program. Since the output of a LD is highly divergent, collimation optics are necessary to guide the lasers into the desired path, fig. 3.2. Aspheric and achromatic lenses that are corrected for spherical aberrations were chosen to collimate the lasers to a diameter of several millimeter. The parallel and perpendicular beam divergences for the blue $\mathrm{LD}$ are: $\Theta_{\|}=10^{\circ}, \Theta_{\perp}=23^{\circ}$. The required focal length $f$ for collimation is calculated by $f=r / \tan \left(\Theta_{\perp} / 2\right)$ where $r=1.5 \mathrm{~mm}$ is the designated beam radius. Accordingly, an aspheric lens with $f \approx 7.3 \mathrm{~mm}$ was chosen. To avoid the light being emitted from the LD clipped by the lenses clear aperture, the NA of the diode has to be smaller than the NA of the lenses $\left(N A_{\text {lens }}>N A_{\text {diode }}\right)$. The DPSSL laser has a beam divergence of just $\Theta=0.43^{\circ}$ (half angle) and requires a much longer focal length of $f \approx 200 \mathrm{~mm}$ for collimation.

To minimize the transmission of thermal and mechanical noise, the lasers are coupled into an optical fiber which is led to the mechanical and acoustical damped center part of the AFM-TIRFM setup. Both lasers are coupled into the same single mode fiber (fig.3.2). Xyz adjustment stages are required for both lasers to overlay the beams precisely before they are combined by a dichroic mirror. The fiber coupling optic is calculated for the longer wavelength. For coupling, the Mode Field Diameter (MFD) of the optical fiber has to be at least equal to the Defraction Limeted Spot (DLS) size $\varnothing_{D L S}=(2 \lambda f) /(\pi r)$ which is generated by the coupling lens. The focal length for an aspheric coupling lens is chosen with $f \leq 13.3 \mathrm{~mm}, f=(M F D \pi r) /(2 \lambda)$. The down focusing of $488 \mathrm{~nm}$ to the MFD is possible with a longer focal length than the one needed for $532 \mathrm{~nm}$. Therefore, the blue laser diode can be also coupled into the fiber by the preliminary determined 
lens. Deploying the lasers through the fiber has two further advantages: First, the fiber will transform elliptic beam profiles as delivered by LD to circular ones. Second, both laser beams are intrinsically aligned on their further beam path through the microscope (fig.3.2). The NA (0.13) of the fiber output and a designated beam diameter of $25 \mathrm{~mm}$ in the central part of the setup require a focal length for collimation of $f \approx 95.8 \mathrm{~mm}$. Furthermore, an aperture was used to select only the center part of the collimated beam. This is done to flatten the excitation profile and to control the illumination spot size.

Because the path of the lasers can be varied with the galvo mirror, it is possible to use the instrument in the laser bright field excitation mode as well as in the TIR excitation mode. Moreover, it is possible to control the evanescent field depth in the TIR mode. Both can be achieved by changing the incidence angle $\theta_{1}$ of the laser beam at the interface between cover glass and sample (fig. 3.1 and section 2.2.1), which corresponds to the objective focal plane (FP). The mechanisms underlying the changes of the incidence angle can be explained by the thin lens approximation. Accordingly, a transversal movement (or rotation) of a ray in the back-focal-plane (BFP) of a lens induces a rotation (or transversal movement) of the ray in the FP of the lens (inset fig. 3.2). BFP and FP are defined by the front and rear focal points which occur when a collimated light beam goes through a lens from one or the other side. Since the BFP of the objective lies inside its lens system, there is no way to access it directly. We gained access to the objective BFP by using a relay lens which creates a conjugated plane to the objective BFP. Therefore, the FP of the relay lens has to correspond to the objective BFP. The principle works the following way: a rotation of the collimated laser beam in the $\mathrm{BFP}$ of the relay lens leads to a translation of the focused beam in the relay lens $\mathrm{FP}$, respectively in the BFP of the objective. A rotation of the collimated beam in the objective FP results. The rotation of the collimated beam in the relay lens BFP was realized by an adjustable rotational mirror (1D Large Beam Diameter Galvo Mirror System, Thorlabs, Germany). The calibration of the galvo mirror system and the incidence angle is described under section 4.1.3. Furthermore, the relay lens facilitates a second important job: it focuses the collimated beam in the objective BFP. This is necessary to create a collimated beam in the objective FP because only a collimated beam results in a constant value for $\theta_{1}$ at the interface between cover glass and sample buffer (fig.3.1). 
The excitation lasers are guided by a dichroic mirror in the objective beam path (405/488/532/635 nm BrightLine quad-edge dichroic beamsplitter, Semrock, USA). It reflects the lasers upwards and transmits the designated dye emissions (transmission bands are shown in fig. 3.3). Beyond the objective the collected detection light is focused by the tube lens with $f=200$. The adapted Image splitter (TwoChannel Simultaneous-Imaging System, Photometrics, USA) allows the detection of different labels and their spectra with a single camera. The transmission and reflection bands of the dichroic mirror and the bandpass filters in the image splitter are shown in fig.3.3.

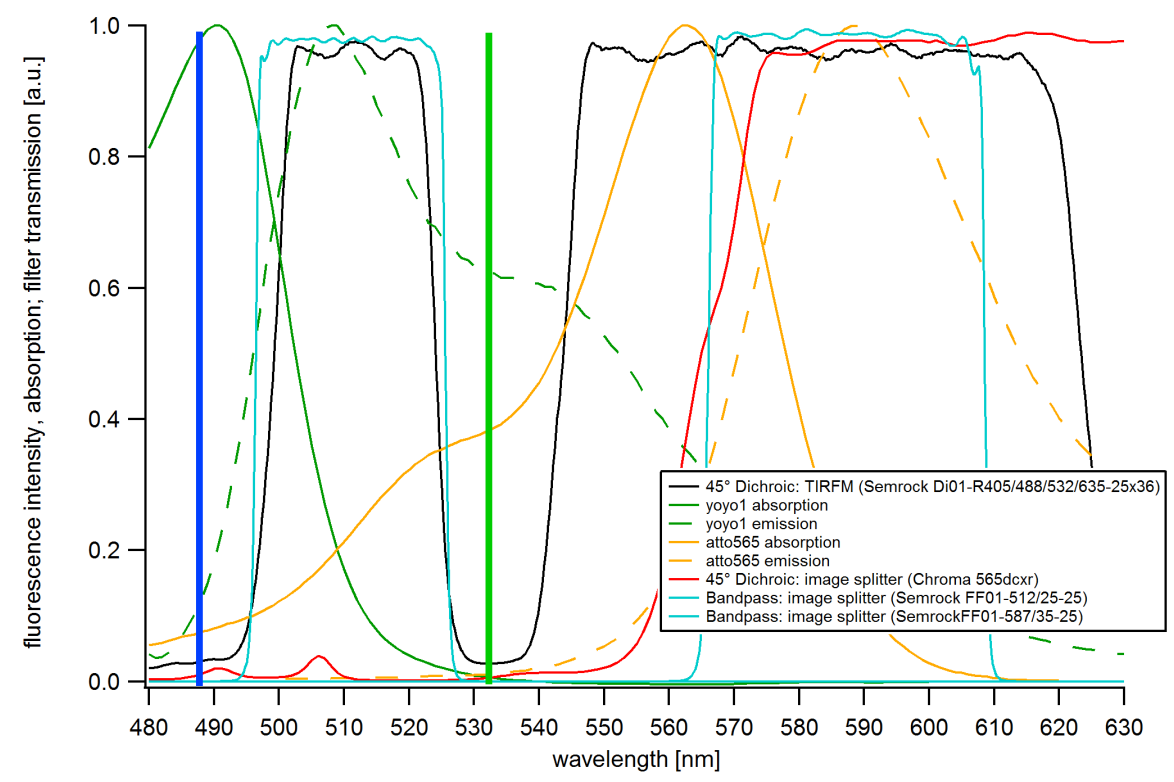

Figure 3.3: Spectral separation in the TIRFM. The dichroic mirror in front of the objective combines the two excitation laser lines $488 \mathrm{~nm}$ and $532 \mathrm{~nm}$ into the objective beam path and allows the emissions of the YoYo- 1 dye and the Atto- 565 dye to transmit. Both dyes are designated for labeling in the following experiments. The dichroic mirror in the image splitter separates the dye emissions so that both can be detected with one camera. To suppress remaining excitation light, two additional band pass filters are placed in the two detection channels. Additional filters to clean up the excitation lines and to block the excitation laser and light originating from the AFM detection system are not shown.

\subsection{Localization microscopy}

The capabilities to resolve two self-luminous points like dye molecules in fluorescence microscopy are limited by diffraction of the emitted electromagnetic waves 
at the aperture of the imaging optics.

The diffraction leads to a radial intensity distribution of the point source image. This response of an imaging system to a point object is described by the Point spread function (PSF). The PSF owns a sharp central peak with small, concentric, and decaying side maxima. The Rayleigh criteria stats that two point sources are resolvable when their intensity peaks lie at the first intensity minimum of the other, figure 3.4. That will be the case if they are separated by a distance of $\Delta l_{\min }$ [11]:

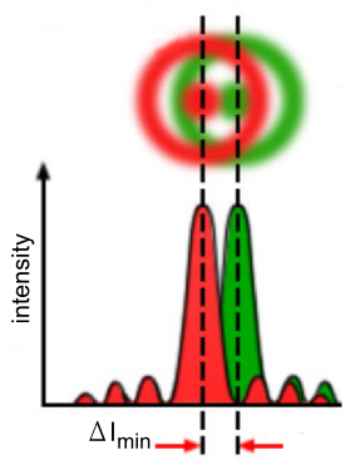

Figure 3.4: Rayleigh criteria, adopted from [51].

$$
\Delta l_{\text {min }}=0.61 \cdot \frac{\lambda}{N A}
$$

Here, $\lambda$ is the emission wavelength and $N A$ the numerical aperture. For the TIRFM presented in section 4.1 .1 and for $\lambda=520 \mathrm{~nm} \Delta l_{\text {min }} \approx$ $213 \mathrm{~nm}$ can be achieved. The intensity distribution center of a single point source, however, can be determined with much higher accuracy. This can be accomplished by fitting a 2D Gauss function to the PSF on a charge-coupled device (CCD) camera chip [52]. The close position of the point source is encoded at the intensity distribution center position which can be derived from the fit function:

$$
f(x, y)=z_{0}+A e^{-\left(\frac{\left(x-x_{0}\right)^{2}+\left(y-y_{0}\right)^{2}}{2 \sigma^{2}}\right)}
$$

$x, y$ are the pixel coordinates and $x_{0}, y_{0}$ are the intensity center coordinates (with sub-pixel accuracy), A is the amplitude and $z_{o}$ a pixel value offset. $\sigma$ is the standard deviation of the 2D Gauss function. It corresponds to a PSF size parameter the Airy disk radius: $r_{\text {Airy }} \approx \frac{\sigma}{0.34},[53]$. The localization error of this position determination method strongly scales with the number of collected photons $N$. Equation 3.3 gives the Standard Error of the Mean (SEM) $\sigma_{n}$ for the method 
[54]:

$$
\sigma_{n}=\sqrt{\frac{\sigma^{2}}{N^{2}}+\frac{a^{2}}{12 N}+\frac{8 \pi \sigma^{4} b^{2}}{a^{2} N^{2}}}
$$

where $a$ is the pixel size and $b$ the standard deviation of the background. The first term is related to the photon noise, the second refers to the finite pixel size, and the third takes background noise into account. Finally, the point source position is determined by: $x_{0}, y_{0} \pm \sigma_{n}$. With a sufficient number of collected photons the error can be arbitrarily small. Practically, the emitted number of photons from dye molecules is limited through bleaching. For single dye molecules distance changes $<5 \mathrm{~nm}$ can be distinguished [55]. However, it is reported that the dipole axis orientation in combination with the use of a high NA objective, effects the minimal achievable errors. So the error can be as high as $10 \mathrm{~nm}$ [56].

The method described for position determination and its error calculations were implemented in a custom written position tracking program (section 6.2). The program was used for the localization experiments presented under section 4.1.4.

\subsection{Fluorescence experiments with single labeled ribosomes}

\subsubsection{Cover glass functionalization and buffer}

In order to conduct AFM and TIRFM experiments with ribosomes, they need to be immobilized on a cover glass surface to prevent diffusion during imaging. Since the AFM tip touches the sample during imaging, the connection must be tight enough to withstand a displacement by the tip. For the experiment circular coverslips (VWR, $25 \mathrm{~mm} \varnothing$, thickness $0.13-0.16 \mathrm{~mm}$ ) were used. First, they were cleaned using the "Potasium Hydroxide $(\mathrm{KOH})$ etching method" as follows:

1. $\approx 10 \mathrm{~g}$ of $\mathrm{KOH}$ pellets are dissolved in $\approx 10 \mathrm{ml}$ of filtered, deionized water with $18.2 M \Omega$ resistance (this water is used in all the following steps as well) and $\approx 200-230 \mathrm{ml}$ of ethanol is added to the solution. 
2. The coverslips are placed in a custom-made support which is then lowered into the glass container with the $\mathrm{KOH}$ solution in it.

3. The container is placed in a bath sonicator for 5 minutes.

4. The coverslips are taken out. The glass container is rinsed and filled with water. The coverslips are sonicated in the water for 2 minutes. This rinsing step is repeated two more times.

Afterwards, the coverslips undergo a silanization step which charges the surfaces positively. Consequently, ribosomes can stick to them. The silanization takes place in multiple steps:

1. $300 \mu l$ of N'-[3-[Trimethoxysilyl]-propyl]diethylenetriamine (DETA) and 30 $\mu l$ of acetic acid are dissolved in a glass container filled with filtered water.

2. The coverslips are placed in the solution and sonicated in a bath sonicator for 5 minutes.

3. The rinsing step described above has to be repeated three times as well.

4. The support filled with coverslips is placed in an oven (preheated to $120^{\circ} \mathrm{C}$ ) for at least 30 minutes for the coverslips to dry.

The chemistry is described in more detail in [57]. The cover glasses were mounted in the Fluid Cell Accessory for the MFP-3D ${ }^{T M}$ AFM (Asylum Research, Santa Barbara, CA). The TAKM 7-buffer solution simulates natural surroundings of the

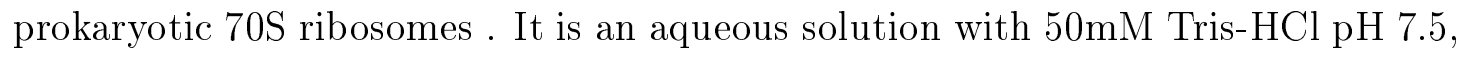
$70 \mathrm{mM} \mathrm{NH}_{4} \mathrm{Cl}, 30 \mathrm{mM} \mathrm{KCl}$ and $7 \mathrm{mM} \mathrm{MgCl}_{2}$ in it.

\subsection{Optical tweezers}

This section is in part reproduced from Bodensiek et. al. RSI 2013 [58]. 


\subsubsection{Beam path design}

To steer the focus (and the trap position) in vertical direction the objective was mounted on a closed-loop objective piezo element (P-721.CDQ with E-625, Physik Instrumente GmbH, Karlsruhe, Germany) with a resolution of $2 \mathrm{~nm}$ (fig. 3.5). To form the trap, we used a single mode fiber coupled LD with an output power of $300 \mathrm{~mW}$. The wavelength of $974 \mathrm{~nm}$ was specifically chosen to minimize the induced damage on biological samples [59]. This needs to be considered because most of the laser light passes through the sample for the duration of the experiment. The laser power measured at the objective aperture is $250 \mathrm{~mW}$. After correction for the objective transmission, this resulted in a power of maximally $200 \mathrm{~mW}$ in the sample. The laser light is coupled into the optical path via a dichroic mirror (fig. 3.5). The laser beam is focused in the sample by a water immersion objective $(1.27$ NA Plan Apo IR objective, Nikon, Japan) which is corrected for infrared light and has a relative high transmission of at least $70 \%$ at $974 \mathrm{~nm}$. The objective aperture is slightly overfilled to maximize the trap stiffness [60]. The 1/e2 intensity profile matches the aperture diameter.

The displacement of the bead with respect to the trap center is monitored with a quadrant photo detector (QPD, $5 \times 5 \mathrm{~mm}$, S5980, Hamamatsu Photonics Deutschland GmbH, Germany). The detector is placed behind the bead in a conjugate plane of the back focal plane of the air-space condenser $(\mathrm{NA}=0.9$, Nikon, Japan). A dichroic mirror behind the condenser separates the near infrared light from the Köhler illumination path of the microscope in the blue range. The axial position of the bead in the trap can be detected through the total intensity on the QPD [27]. Although a single photo diode would be sufficient for the detection of displacements in $\mathrm{z}$ direction, the use of a QPD allows an easy alignment of the detector and also for the displacement detection in the $\mathrm{x}-\mathrm{y}$ plane [24]. The current signals from the QPD are converted into an $\mathrm{x}, \mathrm{y}$, and sum signal using a standard analogue electronic circuit [61]. To maximize the sensitivity of vertical displacements, the QPD is overfilled to achieve an effective NA of $\approx 0.4$ for the collection of the laser light [62]. The $\mathrm{x}, \mathrm{y}$, and sum signals are acquired by a 16 bit AD converter and processed by a FPGA for the feedback control (PCI-7833 R, National Instruments, Austin, TX). Only the sum signal containing the z-position information of the trapped bead was used for further analysis. The data acquisition and the operation of the z-piezo is controlled via a custom written LabVIEW 
program (National Instruments, Austin, TX). 


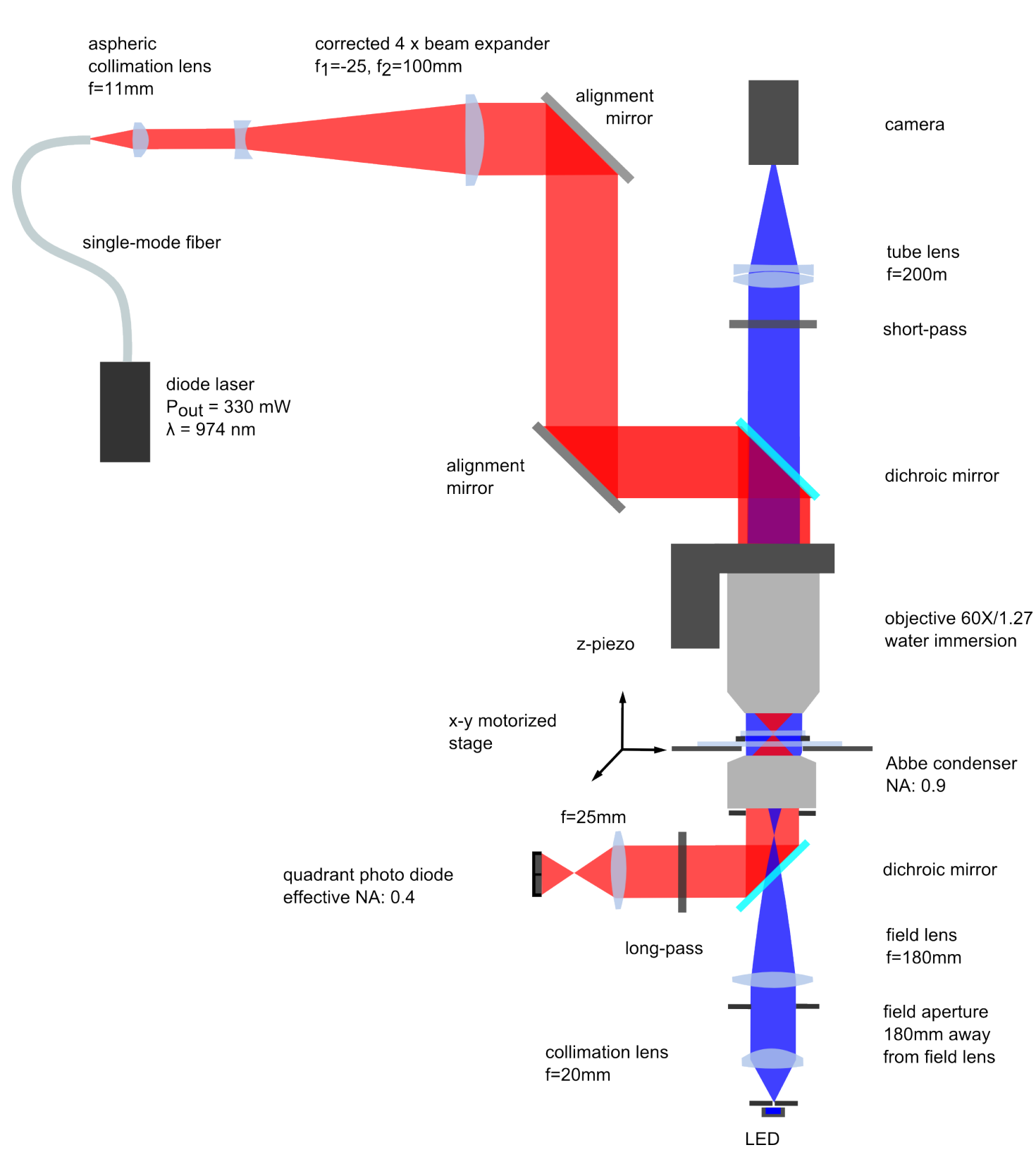

Figure 3.5: Optical design of the vertical optical trap. The divergent laser light coming from the single mode fiber $(\mathrm{NA}=0.14)$ is collimated by an aspheric lens and expanded 4 times by an aberration balanced (longitudinal-spherical) Galileo telescope. The expanded beam is redirected by two mirrors which can be adjusted for aligning the laser with the axis of the imaging path in the microscope. A dichroic mirror, located between the objective and the microscope tube lens, is used to redirect the laser into the back aperture of the objective. The vertical position of the trap is controlled by a z-piezo that moves the objective up and down. To monitor the displacement of the bead from the center of the trap, the laser light is collected by the condenser, coupled out of the optical path via a second dichroic mirror and cast onto the QPD. The illumination source of the microscope consists of a blue LED that is controlled via a standard $100 \mathrm{~mA}$ constant current supply. Imaging is performed by a CCD camera (uEye, IDS-imaging, Obersulm, Germany) placed at $200 \mathrm{~mm}$ from the achromatic tube lens. 


\subsubsection{Optical tweezers alignment}

The following alignment procedure refers to the custom build optical tweezers instrument. Its optical path scheme is shown in fig. 3.5. To align the beam with the optical axis of the instrument an Infrared (IR) viewing card was used (course alignment). First, the objective and the condenser were removed and the dichroic mirror was rotated so that the laser beam went straight through the center of both apertures. After installing the objective and condenser, the Köhler illumination was adjusted. The fine alignment of the instrument started with the observation of the scattered laser light when the objective was focused on the interface between coverslip and buffer. This interference pattern was visible best in an empty sample chamber (glass-air interface). Furthermore, it can be necessary to remove the short pass filter in front of the camera. The two alignment mirrors were adjusted in an iterative process to establish a symmetric interference pattern and to position the trap in the center of the field of view. The symmetry is checked most efficiently while focusing up-and-down through the coverslip. Next, the condenser was aligned in the xy plane (orthogonal to the optical axis) until a symmetric intensity distribution of the laser beam behind the condenser was visible on the IR viewing card. Moving the condenser in $\mathrm{z}$ direction can be required to collimate the laser beam in the detection path. The dichroic mirror under the condenser was adjusted to direct the laser light to the QPD. The QPD was centered in a way that all quadrants received an identical intensity. After this, the two alignment mirrors were adjusted once more to maximize the sum signal of the QPD. This should coincide with the best symmetry of the interference pattern of the coverslip. As final step, a bead was trapped and the trap stiffness was measured. By making fine adjustments of the alignment mirrors it can be tested if the maximum performance has been reached. One or two iterations of the whole procedure may be necessary to achieve the optimal performance. According to our observations the performance remained constant for at least one year of regular use of the instrument after the alignment procedure.

\subsubsection{Sample chamber and beads}

To perform measurements on living cells, a closed chamber was designed. Briefly, a section of parafilm with a diameter of $8 \mathrm{~mm}$ opening cut out was placed onto 
a microscope slide, heated at $55^{\circ} \mathrm{C}$ for $1 \mathrm{~min}$, and pressed firmly against the slide. $17 \mu \mathrm{l}$ of Krebs-Ringer solution $\left(120 \mathrm{mM} \mathrm{NaCl}, 4.7 m M \mathrm{KCl}, 1.2 m M \mathrm{CaCl}_{2}\right.$, $0.7 \mathrm{mM} \mathrm{MgSO}_{4}, 10 \mathrm{mM}$ Glucose, $10 \mathrm{mM}$ Na-Hepes, $p H$ 7.4) containing $0.76 \mu \mathrm{m}$ diameter beads (Bangs laboratories, Fishers, IN) was pipetted into the chamber. Non-functionalized polystyrene beads were chosen since they do not adhere to the cell for the duration of the experiments $(<1 s)$.

\subsubsection{Fitting the periodicity of the interference effect between bead and coverslip}

To measure the periodicity of the approach curves (such as shown in Figure $4.27(\mathrm{a})$ ), the curve was split in segments of $1110 \mathrm{~nm}$ (3 periods) that were fitted with the following function:

$$
f(z)=A \sin \left(2 \pi / z \lambda_{m}+\phi\right)+B+C z
$$

where $A$ is the amplitude, $\lambda_{m}$ is the measured periodicity, and $\phi$ is the phase shift. $B$ and $C$ represent the offset and the slope of the function. The only parameter of interest for us is $\lambda_{m}$. After fitting one $1110 \mathrm{~nm}$ segment the window was moved forward for $100 \mathrm{~nm}$ and fitted again. This procedure was repeated until the whole length of the curve was fitted.

\subsection{Cell indentation}

\subsubsection{Mechanical description of bio material}

Elasticity Elasticity is a material property that represents properties commonly referred to softness and stiffness. 
A purely elastic material will deform under force application but will regain its initial form after release. The characterizing parameter is the elastic modulus also known as Young's modulus $(E)$, which is also frequently used to describe biological material properties. It is defined by: $E=\frac{\text { stress }}{\text { strain }}=\frac{\sigma}{\epsilon}=\frac{F / A}{\Delta l / L}$, where A is the cross sectional area, $\Delta l$ the force $(F)$ induced length change and $L$ the original length (figure 3.6). The elastic response of a material can be visualized in a stress vs. strain plot. There, purely elastic behavior is characterized by a reversible load and reload, fig. 3.7. A purely elastic behavior is e.g. found for a spring

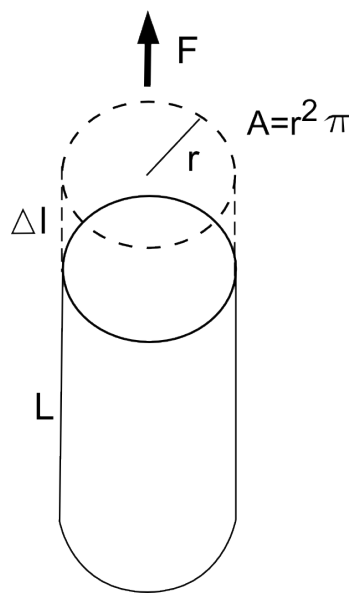

Figure 3.6: Stress \& strain parameters which deforms according to Hooke's Law. A non-reversible stress vs. strain trace is found for non-ideal elastic materials. A non-ideal elastic behavior can be caused by viscous properties of the material of interest. The behavior is than called viscoelastic, fig. 3.7.

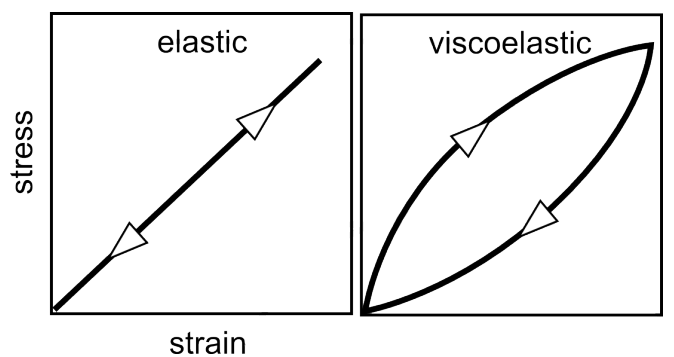

Figure 3.7: Elastic and viscouselastic material response.

Viscoelasticity Viscosity describes the resistance to shearing or extension and originates from friction between neighboring parts with varying velocity. As an example a resting solid plate and a moving plate with a thin liquid film in between can be imagined. The relation between force and induced movement is given by: $\frac{F}{A}=\eta \frac{v}{d}$, where $v$ is the constant velocity of the moving plate, $d$ the thickness of the liquid film, $A$ the plate area, and $F$ the force required to move the plate with $v$. The constant $\eta$ reflects the viscosity of the fluid. In general, viscosity can be expressed as $\sigma=\eta \frac{d \epsilon}{d t}$. Biological materials, especially cells, behave in part viscously. Therefore, deformation time scale (speed) and force (displaced volume) play an important role. Noteably, due to the non-uniform organization of 
cells the contribution of elastic and viscous components in experiments depends on the chosen experimental parameters. Differences in the force vs. indention curves for load and reload indicate the amount of energy that is transformed into a redistribution of cell contents.

One method to model linear viscoelasiticity is the Standard Linear Solid model (SLS model). Here, the mechanical components of a material are simplified by springs and a dashpot. The response to a stress instantaneously applied has an instantaneous elastic component (springs) and a time-dependent component of the

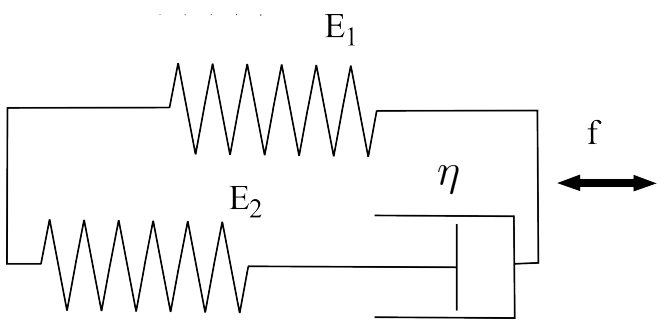

Figure 3.8: The Standard Linear Solid model, a combination of springs and dashpots is used to represent elastic and viscous components.

viscosity (dash pot).

\subsubsection{Contact mechanics}

During most indentation experiments the contact area between indenter and sample is not constant but increases during indentation (fig. 3.9). This leads to a redistribution of the loading force over a larger area during the indention. The force vs. indentation curve will be non linear, even if the material is linearly elastic. The interpretation of AFM-based indentation tests depends on the underlying theoretical model for contact mechanics to determine the Young's modulus. It has been shown that the Hertz contact model is appropriate to determine the Young's modulus for small indentation depths in cell and bio material AFM indentation experiments [63]. For the common indenter sizes, indentations $<0.3 \cdot R_{b}\left(R_{b}\right.$ : spherical indenter radius) deliver consistent results [64]. With the probes that we used on cells, this corresponds to indentations of up to $\sim 500 \mathrm{~nm}$. 
The Hertz contact model describes an idealized case of deformation in which an infinitely large elastic medium is indented with a rigid sphere [65]. The elastic medium can be approximated when force-distance curves are performed on a time scale where viscous contributions are small and the forces measured are dominated by the elastic properties [66]. Furthermore, the model assumes: the material properties of the tip and the cell are isotropic and homogeneous. There is no adhesion between the two bodies and the contact is frictionless. The contact ge-

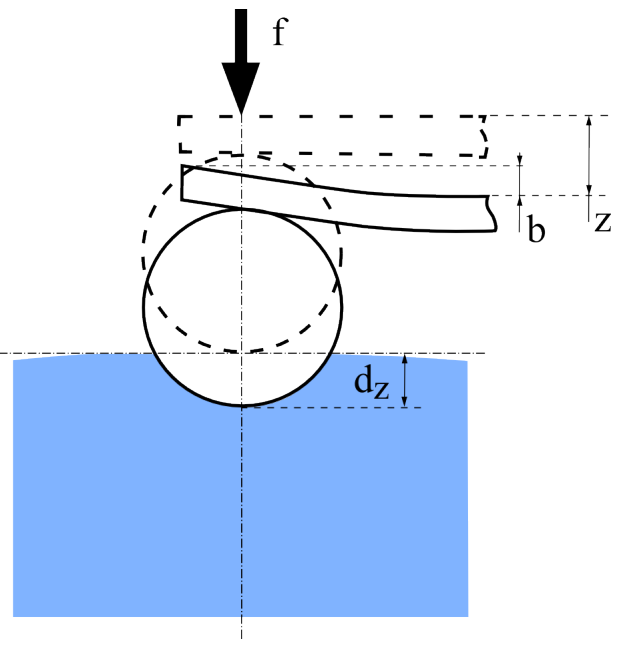

Figure 3.9: Sample and cantilever deformation during a force distance experiment with a spherical cantilever probe.

ometry is axisymmetrically smooth and continuous [67]. The applied force $(f)$ is related to the indentation $\left(d_{z}\right)$ in the Hertz contact model by equation 3.5:

$$
F\left(d_{z}\right)=\frac{4 E}{3\left(1-\nu^{2}\right)} \cdot R_{b}^{\frac{1}{2}} \cdot d_{z}^{\frac{3}{2}}
$$

The indentation is computed from the displacement of the z-piezo $(z)$ minus the bending (b) of the cantilever (fig. 3.9). $\nu$ is the Poisson's ratio; for cells we chose 0.4 [2].

\subsubsection{AFM indentation measurements}

The Young's modulus is derived from the indentation experiments by fitting the recorded force vs. indentation (fig.3.10a) curve with equation 3.5. Fig. 3.10b is displayed for visualization purpose. It reflects the capability of the method to distinguish between cells with a mechanically different response (section 4.5.1). 


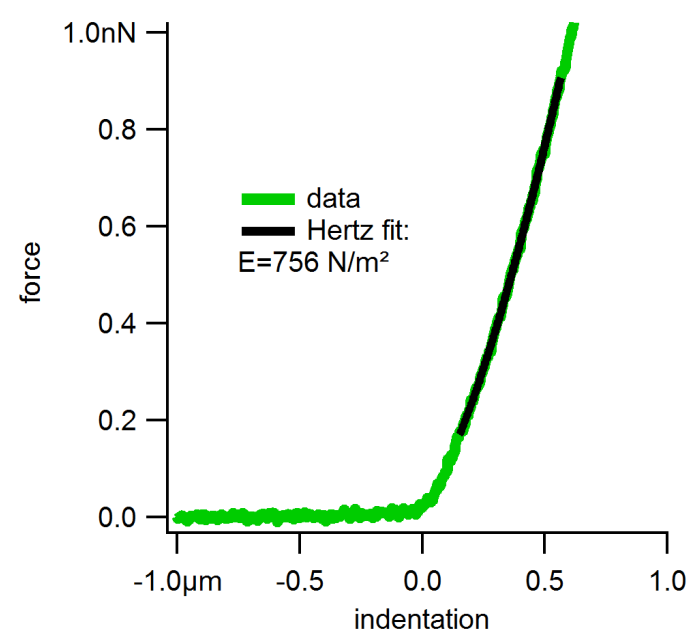

(a) Hertz model fit.

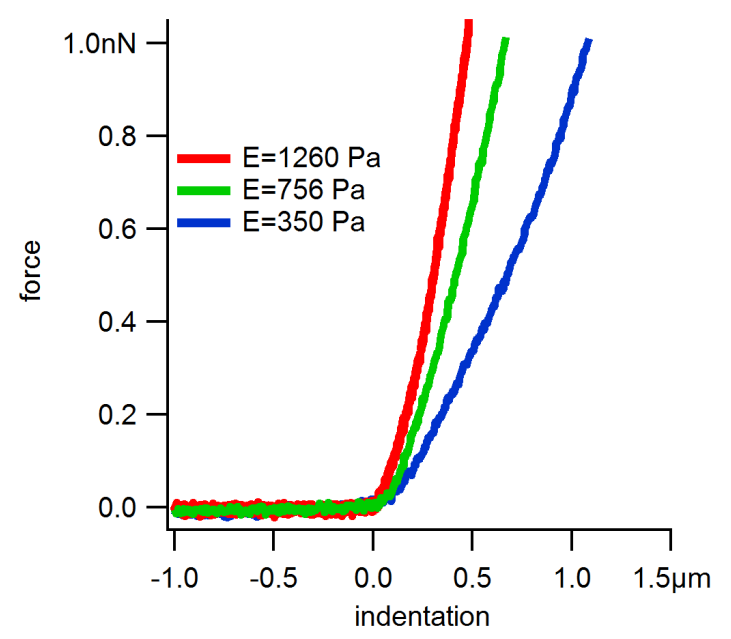

(b) mechanically different cell responses

Figure 3.10: (a) fitting a force vs. indentation relation with the Hertz model. (b) For method demonstration: cells with a mechanically different response (see section 4.5.1). $R_{b}=2.5 \mu m$

\subsubsection{Experimental parameters}

The AFM indentation experiments reported under section 4.5.1 aim to evaluate mechanical differences between $\mathrm{H} 4$ cells, which can be classified according to their fluorescence characteristics, and two control groups. Since a more global effect is expected on the cytoskeleton (section 2.4.1), we decided to distribute the indenting load over an area comprising several $\mu \mathrm{m}^{2}$. Herewith, the contribution of multiple cytoskeleton fibers is averaged and the measurements are less sensitive to local variations in the cell organization. Therefore, a cantilever with a $2.5 \mu \mathrm{m}$ radius borosilicate glass sphere was used. The nominal spring constant of this cantilever is $k=0.035 \mathrm{~N} / \mathrm{m}$. To include the contact mechanics between the probe and the cell, the Hertz model was fitted (section. 3.5.2) between $100 \mathrm{~nm}$ and $500 \mathrm{~nm}$ of indentation. At this relative large deformation we expected to reveal a substantial contribution from the cytoskeleton. The associated forces lie in the range from $\approx 150 p N$ to $1 n N$, where the upper value marks the set point.

At the beginning of the experiments, no information about sub-type dependent differences in viscous-elastic behavior existed. Therefore, the indentation experiments were performed with 3 different indentation speeds $(0.5 \mu \mathrm{m} / \mathrm{s}, 5 \mu \mathrm{m} / \mathrm{s}, 10 \mu \mathrm{m} / \mathrm{s})$. The three sub-types were also compared with a control group which was indented 
at a speed of $(5 \mu \mathrm{m} / \mathrm{s})$. To average out influences of the indention location, every cell was pushed two times with every speed at different positions, always located between the nucleus edge and the cell edge. The derived Young's modulus for one cell at a certain speed corresponds to the mean of the two measurements. 6 data points were acquired for every mutated cell and 2 data points for every control cell, respectively.

The fluorescence sub-type data comprises three different experiments. Hence, for each sub-type three different coverslips were measured with three different cantilevers with an individual calibration for each. The measurements on the cells were performed at the same state of their development (days from seeding) at room temperature within $45 \mathrm{~min}$ after taking the cells out of the incubator.

\subsection{Mechanical Adenovirus unpacking}

\subsubsection{Determining genome spread after capsid breaking}

To compare the genome release of the mature and immature Adenovirus after capsid unpacking, a parameter is needed to describe possible differences. The basic idea is to observe the spatial genome spread. Therefore, the size of the fluorescent labeled genome is detected on a CCD camera chip. The scales of the remaining virus content are expected to vary between $\sim 100 \mathrm{~nm}$ and $\sim 400 \mathrm{~nm}$ after capsid breaking (visual approximations on the basis of AFM images). Those scales are close to the diffraction limited spot size (section 3.2). To describe the diffraction limited spot size, the Airy disk radius is used. It is given by $r_{\text {Airy }}=0.611 \lambda / N A$ [68]. For the presented system it is calculated with $r_{\text {airy }}=213 \mathrm{~nm},(\lambda=520 \mathrm{~nm})$. The pixel size $(16 \mu m \times 16 \mu m)$ of the used camera $\left(i_{X o n_{3}} 897\right.$, Andor, UK) and the 100 times objective reveals one pixel to represent a spatial length of $160 \mathrm{~nm}$ in the image. In sum, these parameters seem to create a spatial under-sampling problem, at least if one wants to threshold pixels and count them to compare the immature and mature genome area.

Hence, we decided to measure the genome spread by using the average width of its intensity image. To do so, the intensity images were fitted with a 2D Gauss function. The standard deviation, giving the width was used to measure the spread 
of the genome. The diffraction limited intensity distribution, the PSF, of a single point source can be approximated by a Gauss function [53] where the standard deviation is related to the airy disc size by: $\sigma \approx 0.34 \cdot r_{\text {airy }}$. For explaining purposes, the labeled DNA can be imagined as one-dimensional arrangement with two bound dye molecules (point sources) separated by a certain distance. Fig. 3.11a shows two point sources seperated by $178 \mathrm{~nm}$ and their Gaussian approximated PSFs. A Gauss fit to their sum intensity distribution delivers $\sigma_{\text {sum }}$ which is broader than the width of a single point source. This illustrates that even if the label separation is smaller than the diffraction limit, it will be detected (since the standard deviation of the total intensity distribution is increased).

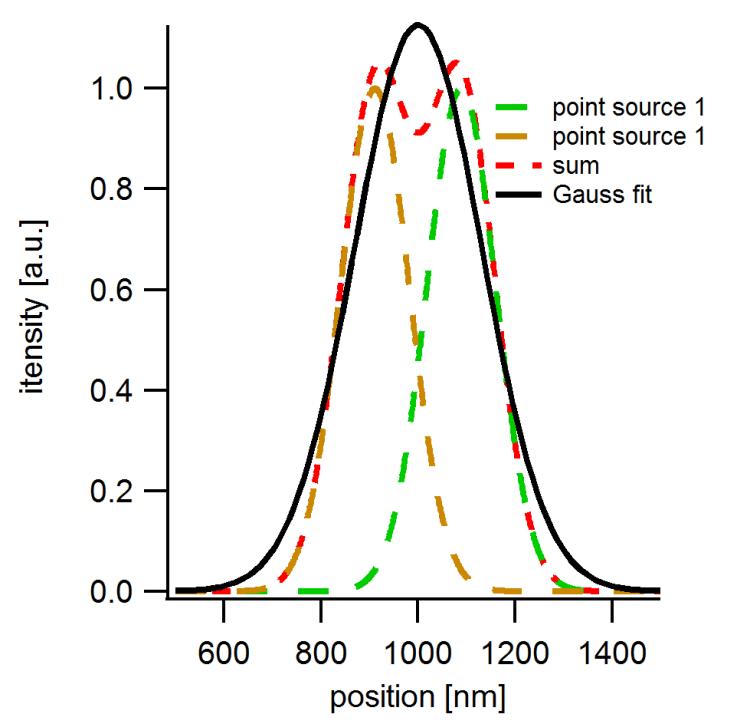

(a) Intensity sum of two point sources.

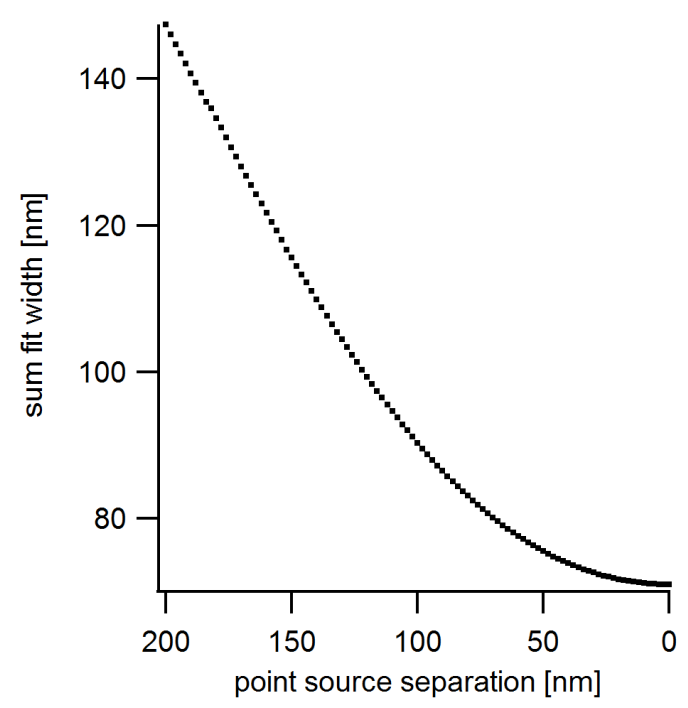

(b) Sum broadening vs. separation.

Figure 3.11: Detection principle of fluorophore separations close to the diffraction limit. (a) The common intensity distribution of two point sources separated by 178 $\mathrm{nm}$. The separation is smaller than the diffraction limit, but it can be detected since it broadens the common intensity distribution. (b) Fitting the sum distribution by a Gauss function and decreasing the separation reveals the relation between width change and separation.

Fig $3.11 \mathrm{~b}$ shows a simulation which illustrates how $\sigma_{\text {sum }}$ depends on the point source separation. For small separations $\sigma_{\text {sum }}$ converges to the standard deviation of a Gauss fit to a single point source of $\sigma=71 \mathrm{~nm}$. Those connections illustrate the effects which allow to recognize spatial separations smaller than the diffraction limit. In reality the genome is a two dimensional arrangement and the situation becomes more complex (which is not regarded in the simplified explanations above). 
To determine the two-dimensional spread of the Adenovirus genome, we fitted a two dimensional Gauss function (equation 3.6):

$$
f(x, y)=z_{0} A e^{-\left(\frac{\left(x-x_{0}\right)^{2}}{2 \sigma_{x}^{2}}+\frac{\left(y-y_{0}\right)^{2}}{2 \sigma_{y}^{2}}\right)}
$$

to the fluorescent spots having appeared after capsid disruption. The means of the width parameters in $\mathrm{x}-\mathrm{y}$-direction $\left(\sigma_{x}, \sigma_{y}\right)$ are used under section 4.5.3 to compare the genome spread.

\subsubsection{Sample dilution and dye concentration}

The virus stock was diluted $\approx 1000 \times$ in a physiological buffer containing $10 \mathrm{mM}$ Tris-HCl $p H$ 8.1, $150 m M \mathrm{NaCl}, 1 \mathrm{mM} \mathrm{MgCl}_{2}$ and $10 \%$ Glycerol as freeze protection. This dilution was divided in $20 \mu l$ portions, refrozen in liquid nitrogen and stored at $-80^{\circ} \mathrm{C}$. For each experiment a $20 \mu \mathrm{l}$ portion was thawed at room temperature for $20 \mathrm{~min}$ and diluted four times in the buffer with $5 m \mathrm{M} \mathrm{NiCl}_{2}$ added. $40 \mu \mathrm{l}$ of this dilution was placed on the Mica substrate and incubated for $10 \mathrm{~min}$. Then, $30 \mu \mathrm{l}$ of this volume were replaced to wash away non-immobilized virus particles. The washing procedure was repeated two more times. The total sample volume was finally increased to $100 \mu \mathrm{l}$. A virus density of $\sim 2$ particles in $25 \mu \mathrm{m}^{2}$ resulted. After mounting and calibration of the cantilever and AFM control imaging of one virus particle, we added a DNA specific dye (YOYO-1 Iodide, Life Technologies, USA) to the sample volume. So, a concentration of $300 n M$ was established. The bis-intercalating dye has an absorption maximum at $\lambda_{\max }=491 \mathrm{~nm}$ and an emission maximum at $\lambda_{\max }=509 \mathrm{~nm}$. If bound to double stranded DNA, it will increase its fluorescence 3000 times (spectra in fig. 3.3).

\subsubsection{Sample support and immobilization}

For AFM imaging and TIRFM the virus capsids must be immobilized so that they cannot be moved by lateral force exerted by the cantilever tip during scanning. The lateral forces in tapping mode AFM are minimized compared to contact 
mode imaging. Notably, immobilizing the virus particles on the silanized surfaces, described under section 3.3.1, turned out to be not sufficient. To prevent the occasional disappearance of the virus particles during scanning, we decided to use Mica substrates which are known to work well from preliminary AFM-only experiments. To absorb the virus particle from the buffer on the Mica, $5 m M \mathrm{NiCl}_{2}$ must be added. Müller et al. [69] state the following absorption mechanism: The micas freshly cleaved negative surface charge density gives rise to an accumulation of counter ions. This results in an electro-static double layer. Furthermore, macromolecules (capsid) are charged as well due to a dissociation of functional groups at their surface. Again, this promotes the creation of an electro-static double layer. The counter ions create a diffusive layer and when both double layers approach one another, they begin to overlap. An increase of the osmotic pressure and the double layer force results. The addition of $\mathrm{NiCl}_{2}$ is necessary to decrease the thickness of the diffusive layer (represented by the Debye length). When it is decreased to a sufficient extend the capsids can diffuse so close to the surface that the attractive Van der Waals forces dominate. At this point the capsids are pulled into contact and are sufficiently absorbed on the mica.

The use of Mica substrates is a trade off between the immobilization advantages and perfect optical imaging performance. The non-perfect imaging performance results from the birefringence and the range of refractive indices of the mica. The birefringence plays a minor role since the mica is cleaved to a minimal thickness of roughly some microns. The refractive index mismatch inbetween the cover glass sample buffer degrades the optical focus of the objective. In total, both effects increase the minimal achievable focus spot size and, therefore, decrease the resolution. The intensity spot width (Gauss fits standard deviation) of a 100 $\mathrm{nm}$ fluorescent bead on normal cover glass is determined with $148 \mathrm{~nm}$. On the Mica substrate this width is increased by $65 \mathrm{~nm}$ (section. 4.1.4.1). Due to the lack of alternatives, we finally decided to use mica as sample support (Muscovite Mica, V-1 Quality, Science Services, Germany). 
For a practical usage, the thin cleaved Mica must be supported by a solid structure. The mica discs are glued on microscopy cover glasses which fit in the liquid-cell holder of the AFM. A transparent UV hardening glue (NOA65, Norland Products Inc., USA) was used. The creation of TIR requires the transition from a medium with a high refractive index to a medium with a low refractive index, (section 2.2.1). To create the TIR at the interface between buffer and

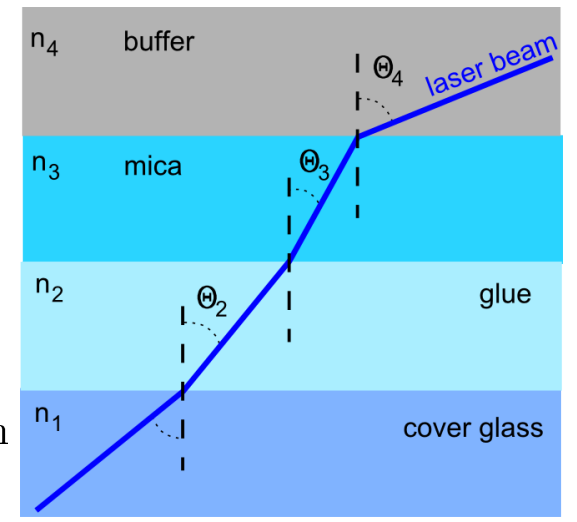

Figure 3.12: Refractive indexes in the sample support.

mica the following connections between the refractive indices of the complete sample support components are required (fig. 3.12): $n_{1} \leq n_{2} \leq n_{3}>n_{4}$ or expressed as refraction angles: $\theta_{1} \geq \theta_{2} \geq \theta_{3}<\theta_{4}$. The refractive index of the glue $\left(n_{2}=1.52\right)$ fulfills these requirements.

The assembly of the sample-supports includes the following steps: The Mica has to be precleaved with a scalpel as thin as possible. $12 \mathrm{~mm}$ discs have to be cutted from the precleaved sheets with a hollow puncher. The discs must be glued to the cover glasses. Hardening takes place under UV light. The glue edge around the disc is removed with a scalpel. Before the experiment, the Mica layers have to be repeatedly cleaved by stripping off layers with tape. Empirically, the mica disc can be cleaved around four times before the minimal thickness is reached.

\subsubsection{AFM and TIRFM detection}

For AFM virus imaging and pushing, a pyramidal cantilever with a $\kappa=0.05 \mathrm{~N} / \mathrm{m}$ and a tip radius of $15 \mathrm{~nm}$ was used (OMCL-RC800PSA, Olympus, Japan). Imaging was performed in tapping mode with a free amplitude around $800 \mathrm{pm}$ and a set-point roughly $100 \mathrm{pm}$ smaller. This reduction corresponds to a force set-point of $\sim 40 p N$. The cantilever driving frequency was set to approximately $23 \mathrm{kHz}$. The integral gain was set roughly to 70 and the proportional gain to 0.05 . The image size was $400 \mathrm{~nm} \times 400 \mathrm{~nm}$ with $128 \times 128$ points $\&$ lines and a scan speed of $1 \mu \mathrm{m} / \mathrm{s}$ (higher speeds tend do damage the virus capsid). 
Before the force curve was applied on a virus capsid, a force curve close to the virus location was performed on the substrate. This was done to find the surface level and to adjust the start distance for the push on the virus. The start distance was set to $400 \mathrm{~nm}$ above the substrate so that after centering the tip above the virus capside, both were separated by $\sim 320 \mathrm{~nm}$.

The approach speed used was $400 \mathrm{~nm} / \mathrm{s}$, and the force set point $\sim 10 \mathrm{nN}$. For TIRFM imaging an exposure time of $100 \mathrm{~ms}$ was chosen in combination with a electron multiplying gain of $300 \%$. The laser excitation intensity was adjusted to $0.34 \mu \mathrm{W} / \mu \mathrm{m}^{2}$ at a TIR angle of $64^{\circ}$. 


\section{Results and Discussion}

\subsection{The combined TIRFM-AFM setup}

\subsubsection{Design}

In this section the mechanical combination of the AFM and the custom made TIRFM is described. The detailed design of the TIRFM optical beam path is given under the method section 3.1. The commercial AFM (MFP-3D, Asylum Research, USA) available in our lab at the beginning of this project served as the starting point for the realization of the combined AFM-TIRFM setup. The central hardware components of this microscope are the xy-scan piezo unit and the AFM head with the z-scanner. The sample is mounted into the sample holder in the xy-scan table and the cantilever is mounted into the cantilever holder in the AFM head. Scanning requires the movement of the sample (xy-direction) as well as the movement of the cantilever (z-direction). To damp external vibrations the AFM rests on an active isolation table (Nano30, Accurion, Germany). In the common AFM-working arrangement the cantilever tip points in gravity direction. The AFM design allows optical access to the sample only from below (fig. 4.1). Therefore, the TIRFM had to be built as an inverted microscope. Additional space between the xy-scan unit and the noise-isolation table was necessary for the realization and was obtained by raising the xy-scan unit by a post assembly. 


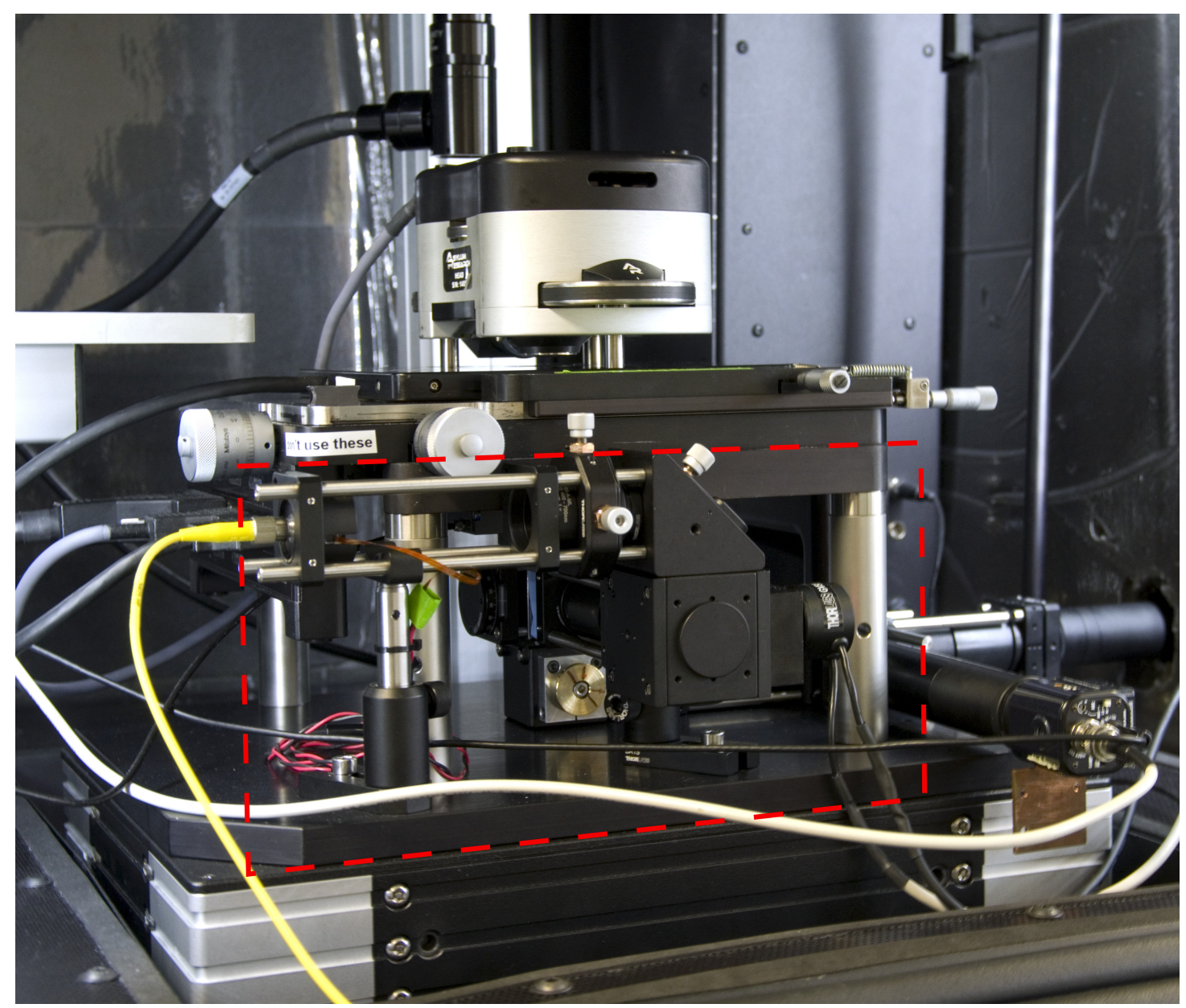

Figure 4.1: Mechanical realization of the central structure to combine both microscopes. Starting from above there is the AFM-head, containing the AFM z-scan piezo. The sample holder is located in the plane of the xy-scan table (under the head). The following TIRFM components are located inside the marked region (red box): objective mounted on another z-focus piezo element, dichroic mirror, galvo mirror system, excitation laser coupling, additional optical components. A detailed optical scheme is given under section 3.1. The additional space required for these components is created by placing the AFM xy-scan table on posts.

Since the AFM parts involved in scanning are extremely sensitive to acoustic noise, the whole AFM (besides the electronics controller) is enclosed in an acoustic damping hood, fig. 4.2. The temperature inside the hood can be adjusted and held constant by a temperature controller. 


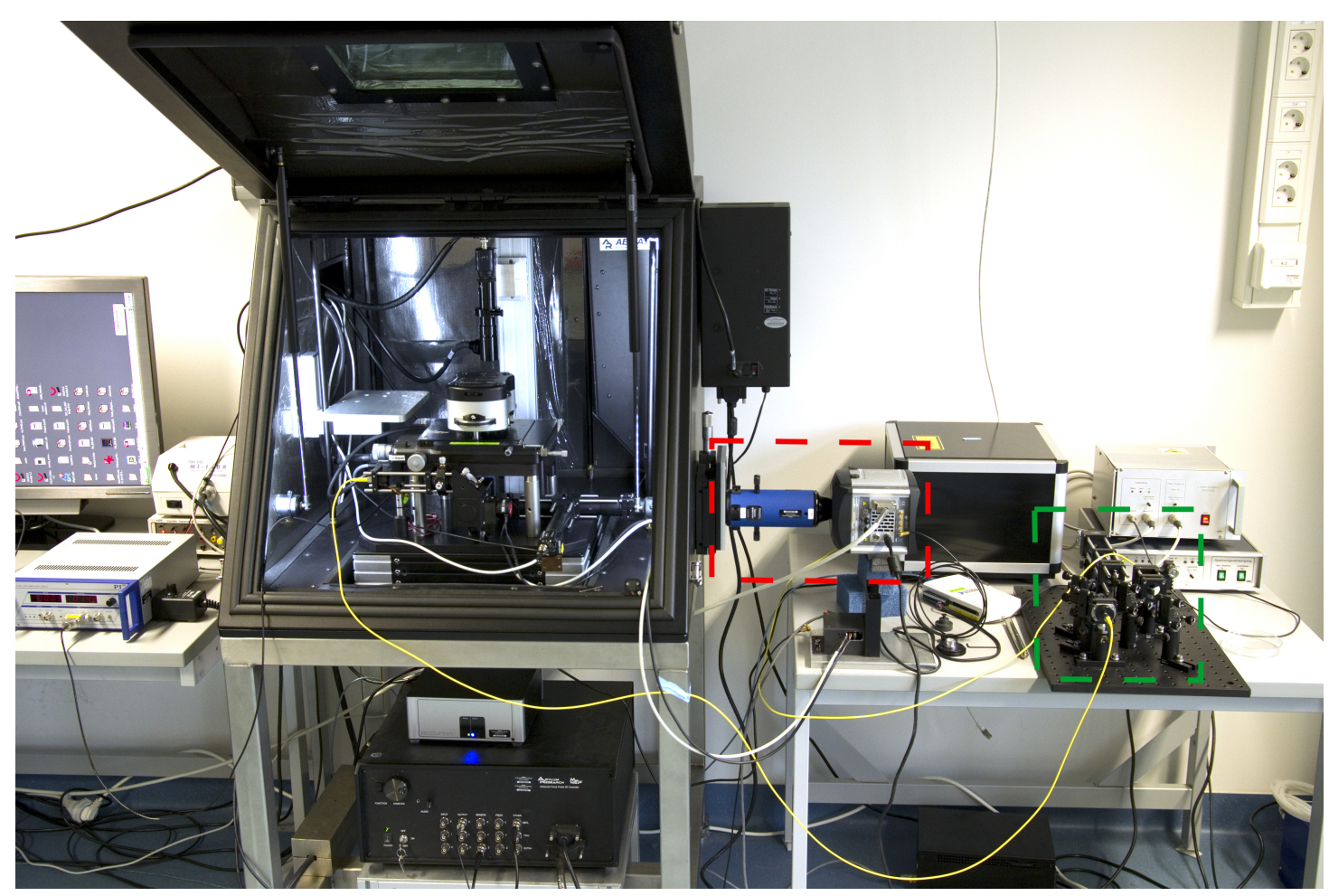

Figure 4.2: A photograph of the complete instrument shows the mechanical realization of the experimental setup for the combined AFM-TIRFM. The excitation lasers (green box) and the camera (red box) are placed outside the acoustic damping hood.

To minimize thermal influences and mechanical noise coupled to the central parts of both microscopes, the excitation lasers and the camera of the TIRFM are placed outside the hood. Also the connection between both parts is not mechanically rigid. This will be explained below.

\subsubsection{Noise uncoupling}

Since a mechanical connection of the image splitter and the camera with the central microscope structures would induce unwanted mechanical noise, these components are mechanically decoupled. The noise picked up with an AFM cantilever in the AFM-TIRFM combination with and without a rigid mechanical connection between camera and AFM is shown in fig. 4.3. 


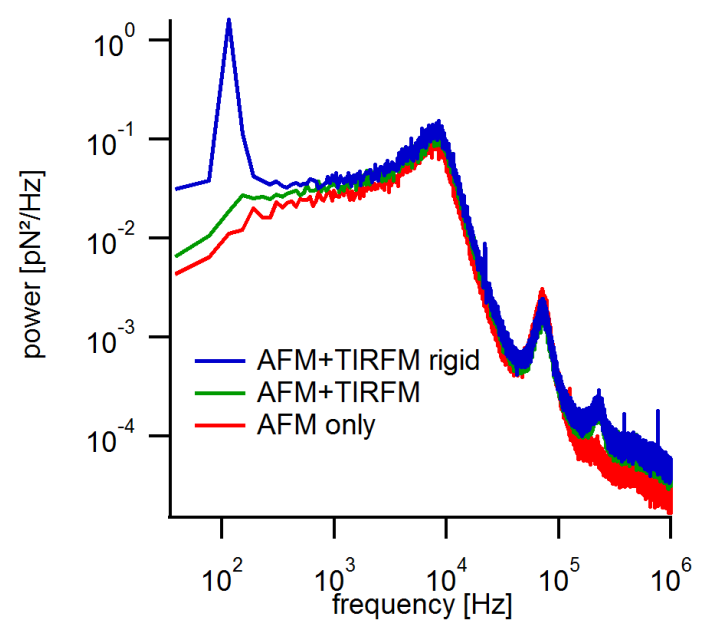

(a) PSD

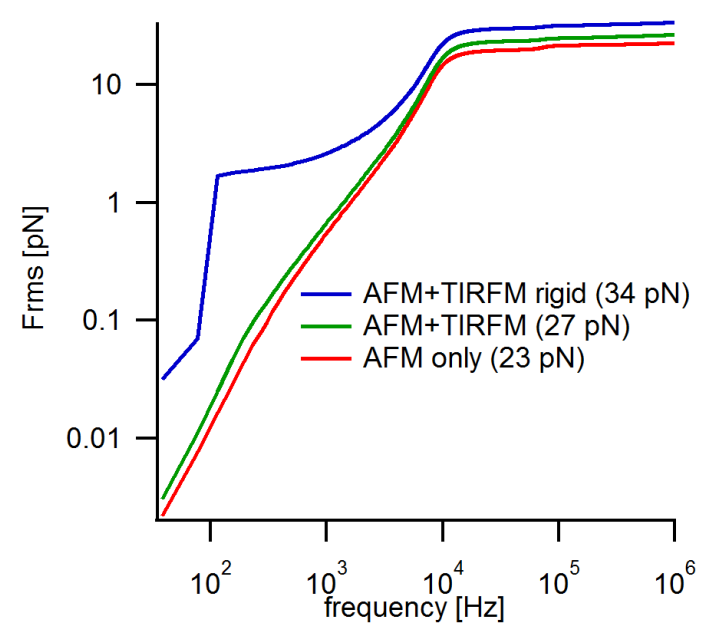

(b) RMS force noise

Figure 4.3: Instrument noise. (a): Cantilever noise in liquid environment depicted as PSD. The used cantilever (BL-RC150VB-HW, Olympus, Japan) is placed $5 \mu m$ away from the interface. (b): The RMS force noise of the cantilever. The noise level of the AFM-TIRFM combination with the rigid connected image splitter / camera is the highest one and visible in an additional peak around $100 \mathrm{~Hz}$ (a) what leads to a higher noise level especially in the frequency range $<5000 \mathrm{~Hz}(\mathrm{~b})$. The noise level with the non-rigid connection is just slightly higher than the noise level without TIRFM.

The cantilever noise is determined by calculating the PSD from the cantilever fluctuations as described under section 2.1.1. The RMS force noise of the cantilever is calculated by the integration of the PSD according to equation 4.1 [70]:

$$
F_{R M S}=\sqrt{\int_{f_{1}}^{f_{2}} F^{2}(f) d f}
$$

The presented results show that the mechanical decoupling decreased the noise in the combined setup. The main noise source is the cooling fan of the Electron Multiplying Charge Coupled Device (EMCCD) camera. It is possible to switch off the cooling fan for several seconds and to reduce the noise that way. Practically, it is necessary to discharge the heat generated by the thermal electric cooling of the EMCCD and to hold it constant at $-80^{\circ} \mathrm{C}$ to ensure low levels of thermally generated charges (noise) in the pixels. Liquid cooling as a possible alternative 
turned out to create even more vibrations than the air cooling due to the waterpump. Several EMCCD cameras were used throughout the experiments. For the respective fluorescence experiments presented later: $i X$ on $_{3} 860$, Luca S $658 \mathrm{M}$, $i X n_{3}$ 897, Andor, UK. The adapted Image splitter (Two-Channel SimultaneousImaging System, Photometrics, USA) allows the detection of different labels and their spectra with a single camera.

\subsubsection{Controlling the depth of the evanescent field}

In a combination of AFM and TIRFM, the cantilever tip is placed inside the excitation and emission volume of a fluorescence labeled target. This causes interactions of the excitation light with the cantilever and its tip which include scattering, reflection, and even excitation of surface plasmons [12]. These effects result in additional light competing with the light of fluorescently labeled targets. Even with careful chosen filters, this additional light can outshine light emissions from single fluorophores (see section 4.2.4). This is especially pronounced in the non TIR excitation regime due to the excitation laser illuminating the whole tip and even parts of the lever. In the TIR mode itself it is of interest to investigate the relation of the penetration depth of the evanescent excitation field and the tip related background light. As described und section 2.2.1, the field depth depends on the incidence angle. To control the angle, the collimated beams need to be able to rotate in the objective FP. This is realized by the principles explained under the method section about the TIRFM beam path design, 3.1. The central part is an adjustable rotational mirror (1D Large Beam Diameter Galvo Mirror System, Thorlabs, Germany) rotating in a conjugated plane (created by a relay lense) to the objective FP.

All optics and the large amount of glass between the galvo mirror and the TIR interface make it necessary to calibrate the galvo mirror system, to find the relation between the incidence angle and the steering voltage delivered to the mirror controller. To measure the angle between objective optical axis and laser beam without refraction disturbance, a glass half circle $(\mathrm{r}=15 \mathrm{~cm}$, thickness $=1 \mathrm{~cm})$ was placed over the objective with the function of a protractor. Because the objective front lens, immersion oil, and glass half circle have the same refractive index, the laser beam propagates at its incidence angle through the glass half circle. To mea- 
sure the angle, the visible laser beam spot was observed at the edge of the glass plate which is equipped with an angle scale. The steering voltage was scanned for the possible angle range in one degree steps. The derived calibration curve is shown in fig. 4.4. It is stored in a custom written Labview program to allow an easy access to the different angles. Furthermore, the angles can be synchronized with the excitation lasers and the camera exposure. Since the NA is defined by the Sine function of the angle between the optical axis and the light acceptance cone, it is not surprising that the relation between steering voltage and angle is similar to a Sine function. Due to the optics disturbances the relation between the incidence angles and the steering voltage is better described by a 3rd order polynomial.

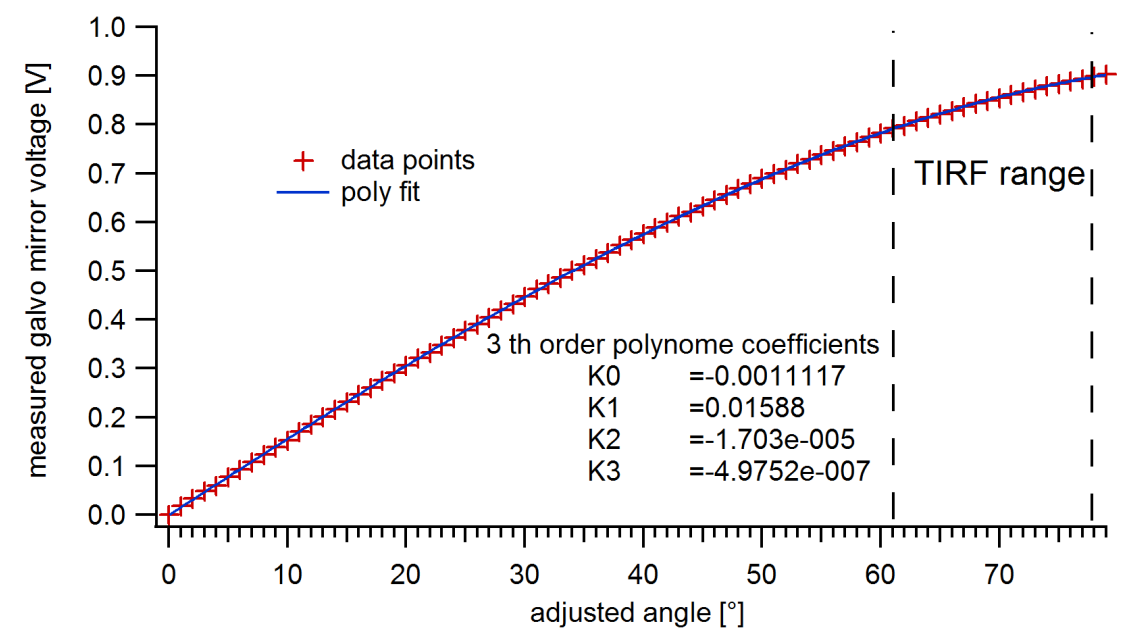

Figure 4.4: Calibration curve for the galvo mirror system. The relation between galvo mirror steering voltage $u$ and excitation laser incidence angle $\theta_{1}$ is determined experimentally. The definition of the objective NA leads to an explantion of the similarity between the steering signal and a Sine function. The deviations are caused by the massive optics and possible nonlinearities of the galvo mirror system. Finally, a 3 rd order polynom $\left(u\left(\theta_{1}\right)=k_{0}+k_{1} \theta_{1}+k_{2} \theta_{1}^{2}+k_{3} \theta_{1}^{3}\right)$ is used to control the angle via a custom written labview program.

\subsubsection{TIRFM localization accuracy}

The localization method for single self-luminous point sources and its accuracy is explained under section 3.2. On the combined TIRFM-AFM setup mechanical uncoupling between the central instrument and the camera potentially reduces the achievable localization accuracy. The uncoupling can cause that the collimated 
beam emanating from the objective enters the tube lens off-axis. The off-axis effect can vary over time since the active damping table continuously makes small height corrections. Here, off-axis refers to a parallel shift of the objective optical axis to the tube-lens optical axis of up to $\sim 350 \mu \mathrm{m}$ and / or an angle between both axis of up to $\approx 0.2^{\circ}$. Both values are estimations from the possible piezo strokes and their points of application. This effect can shift the position of a point source image on the CCD detector. To investigate the remaining localization accuracy, the position of a fluorescent bead with a radius of $50 \mathrm{~nm}$ was determined every 0.5 $s$ over a time of one minute (fig. 4.5). The position tracking was performed with the custom written program (section 6.2). The bead was immobilized on silanized cover glass in deionized water containing $5 \mathrm{mM} \mathrm{MgCl}_{2}$. It was excited under a TIR angle of $74^{\circ}$ with $0.79 \mu \mathrm{W} / \mu \mathrm{m}^{2}$ and detected with a camera exposure time of $50 \mathrm{~ms}$.

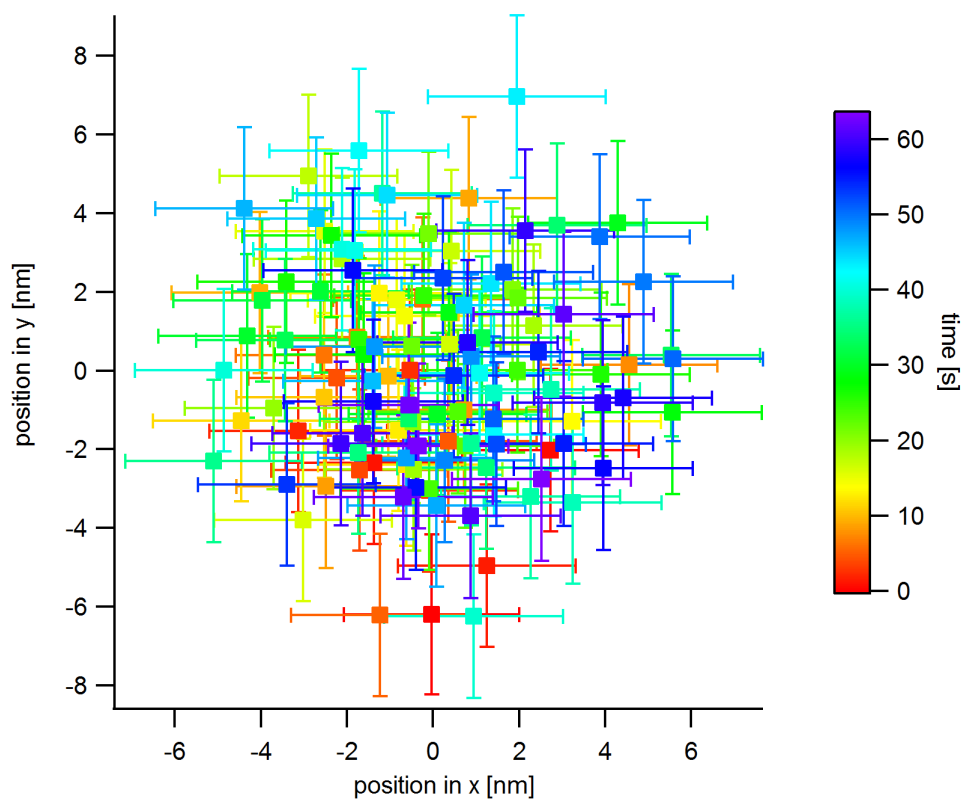

Figure 4.5: Fluorescence microscopy localization accuracy with a mechanically decoupled camera. An off-axis effect between optical axis of objective and tube-lens can occur due to the mechanical decoupling. Therefore, the accuracy when using the fluorcence localization method described under section 3.2 was investigated. Tracking of an immobilized fluorescent bead reveals a certain spread of the measured positions. The error bars are the SEMs of the position determination method itself. The total error in the combined microscope can reach values of up to $\sim \pm 8 \mathrm{~nm}$.

The error bars reflect the SEMs (calculated with equation 3.3) of the position determination method itself. The off-axis influence during the image acquisition time 
is neglected. The average method SEM is $\sigma_{n} \approx \mp 2 n m$. If bead position variations and variances of the fitting method are assumed to be very small, the main position spread will be caused by the off-axis effect. When using the fluorcence point source localization method described under section 3.2 the total position determination error in the combinded TIRFM-AFM can reach values of up to $\sim \pm 8 \mathrm{~nm}$. Going one step further and evaluating the capabilities to measure translations of a point source in the xy-plane leads to a test series with defined position changes. In the following experiment, the positions of a bead were changed in step sizes from $10 \mathrm{~nm}$ to $100 \mathrm{~nm}$ by moving the AFM xy-stage. The stage was driven by piezo actuators with a feedback loop and an accuracy of about $1 \mathrm{~nm}$. The step size is determined by subtracting the previous position from the post-step position. The results are shown in fig. 4.6.

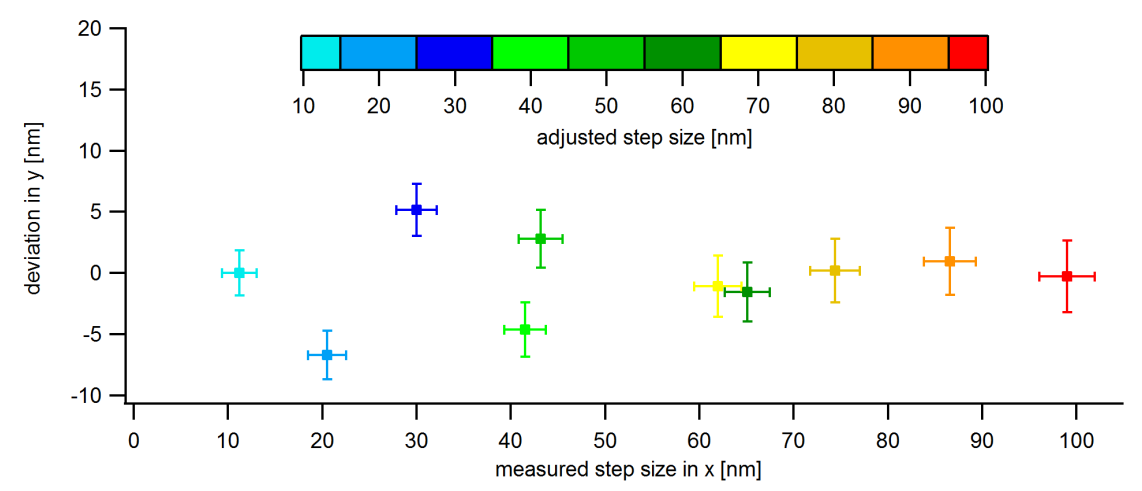

(a) Deviation between adjusted and measured step size in $\mathrm{x}$ direction.

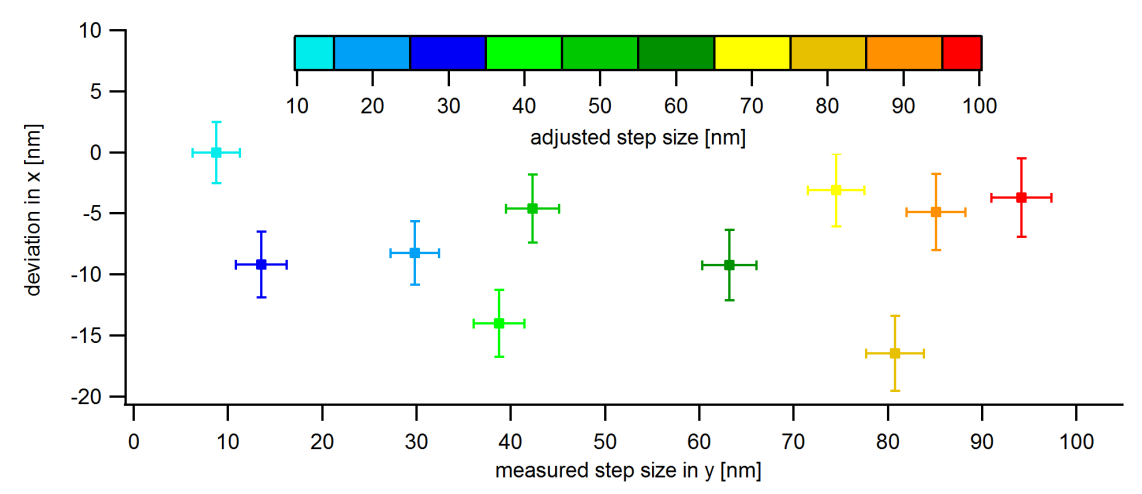

(b) Deviation between adjusted and measured step size in y direction.

Figure 4.6: Capabilities to measure translations of point sources. The low noise configuration (no rigid connection between tube-lens and objective) makes the position determination of fluorescent point sources less accurate. 
Again, the error bars symbol the SEMs of the method including the error propagation in the subtraction. The biggest deviation measured from the adjusted step size is $10 \pm 2.9 \mathrm{~nm}$ in $\mathrm{x}$-direction and $17 \pm 3.2 \mathrm{~nm}$ in y-direction. For the total deviation in the xy-plane the geometric summation returns $20 \pm 4.3 \mathrm{~nm}$. Consequently, smaller displacements are not reliably resolvable in this system configuration.

\subsubsection{Longitudinal depth of field}

The diffraction limited intensity image of a point source has a certain radial width expressed by $r_{\text {Airy }}$, (section 3.2). This size parameter increases with increasing defocusing. Close to the focus center a small change is apparent according to its parabolic behavior. In Gaussian beam optics this region is referred to as the Rayleigh Length. In Microscopy a similar range is defined for the visual depth of field $\left(z_{\text {depth }}\right)$ [71]:

$$
z_{\text {depth }}=\frac{\lambda_{0} n}{N A^{2}}+\frac{e n}{M N A}
$$

where $n$ is the refractive index of the immersion media, $M$ the lateral magnification of the objective, and $e$ the smallest distance that can be resolved by a detector placed in the image plane of the microscope objective. Equation 4.7 should not be confused with the resolution criteria in $\mathrm{z}$ direction. It refers to the region in which an image is perceived as focused. For the TIRFM we get a result of $z_{\text {depth }}=382 \mathrm{~nm}$. To experimentally characterize and use the relation between $r_{\text {Airy }}$ and the z-focus position, the spot size (standard deviation $(\sigma)$ of a Gauss fit to the imaged intensity distribution) is measured at different positions. Therefore, an immobilized fluorescent bead with a radius of $50 \mathrm{~nm}$ was imaged during a scan of the focus in z-direction. The scan was realized by moving the piezo mounted objective. The spot size of the bead image was determined every $50 \mathrm{~nm}$. The results are shown in figure 4.7 : 


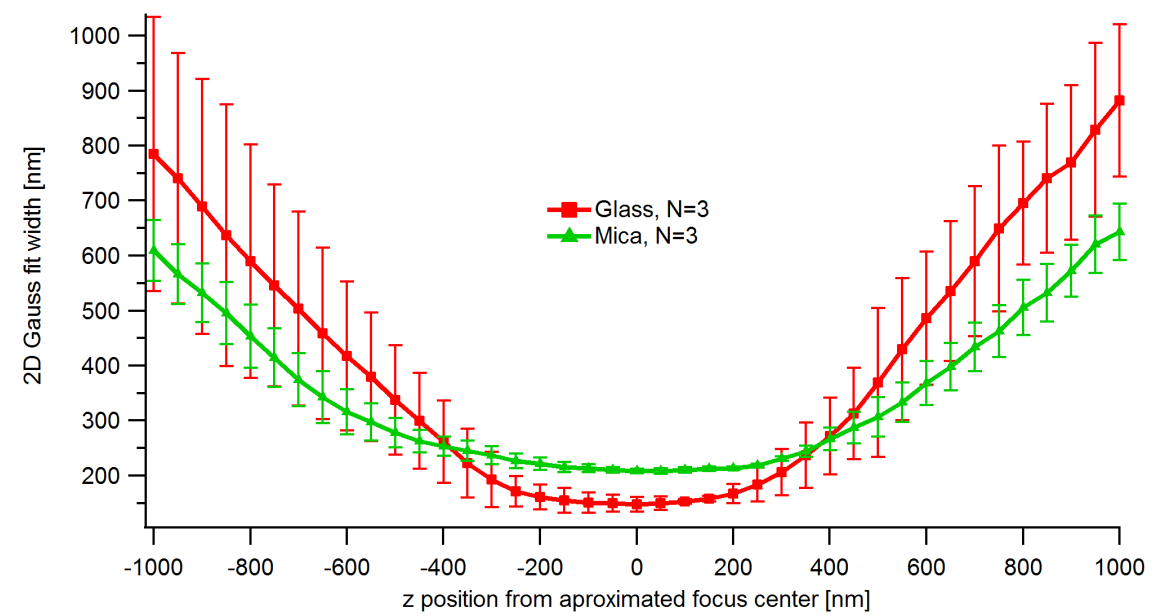

Figure 4.7: Spot size of a $100 \mathrm{~nm}$ diameter fluorescent bead vs. z-focus position. Greater defocusing increases the spot size of an imaged bead. On cover glasses the dependence was investigated to implement a z-direction tracking possibility in the custom written xy-position tracking program (section 6.2). Moreover, the relation was also investigated on Mica substrates which were used in the experiments of section 4.5.3. The refractive index mismatch on the Mica substrate and its birefringence increase the spot size.

Since the spot size parameter used here equals the standard deviation of the fit, the minimal spot size in fig. 4.7 seems to be smaller than the diffraction limited spot size. To compare $\sigma$ with the diffraction limited spot size the Airy disc radius must be used which stands in the following relation to the standard deviation, $\left(r_{\text {Airy }} \approx \frac{\sigma}{0.34}\right)$. On a cover glass, the measured spot size in focus is $\sigma \approx 148 \mathrm{~nm}$. Compared to the theoretical value for a single point source of $\sigma \approx 71 \mathrm{~nm}$, this value is much higher. The deviation is caused by the convolution of the multiple point source PSFs, that result from the $100 \mathrm{~nm}$ beads. The derived spot size focus position relation is implemented in the custom written position tracking program described under section 6.2. Together with the xy-plane localization (section 3.2), this allows a 3-dimensional tracking of the above specified beads. Although the position sensitivity is much smaller in z-direction than in the xy-plane and the $x y-$ accuracy for the defocused image is reduced, this implementation can be used to estimate the 3-dimensional drift of the microscope. The results of an experiment with images taken every $30 s$ over a time of thirty minutes are shown in fig. 4.8: 


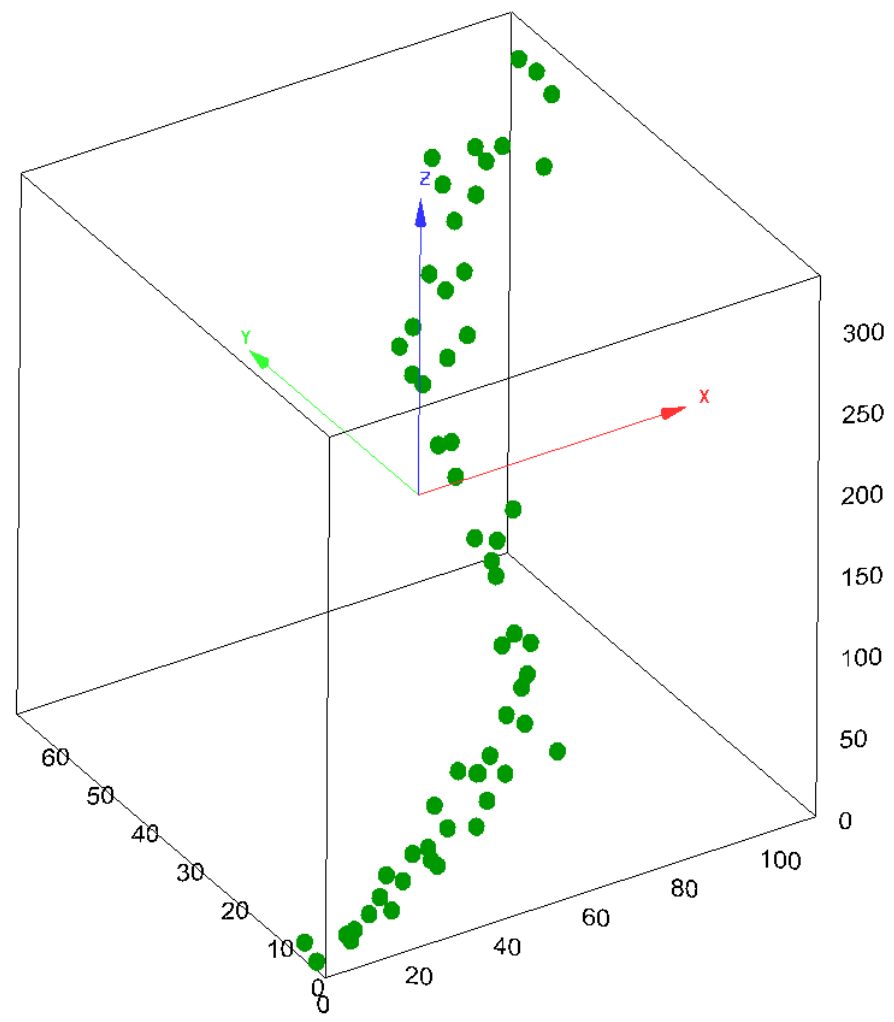

Figure 4.8: Three dimensional microscope drift within 30min. The tracking in z-direction is facilitated by including the spot size vs. z-position relation for a fluorescent $100 \mathrm{~nm}$ bead in the custom written tracking program (section 6.2). Axis units are given in $\mathrm{nm}$.

Values of $100 \mathrm{~nm}$ drift in $\mathrm{x}, 60 \mathrm{~nm}$ in $\mathrm{y}$, and $300 \mathrm{~nm}$ in $\mathrm{z}$ direction indicate an acceptable drift behavior of up to $10 \mathrm{~nm} / \mathrm{min}$ (compare also fig. 4.20).

The same z-scan experiment was performed on the mica substrates described under section 3.6.3, which were designated for the use in the mechanical Adenovirus unpacking experiments (section 4.5.3). The refractive index mismatch and the materials birefringence cause a focus degradation which increases the spot size to $\sigma \approx 213 \mathrm{~nm}$ (fig. 4.7). This indicates that the effective NA is decreased, which increases the depth of field and makes the spot size less sensitive for imperfect focusing. In z-direction the spot size changes by less than ten percent over a distance of $\sim 400 \mathrm{~mm}$. 


\subsubsection{TIR excitation decay}

A key feature of TIRFM is the evanescent excitation field emanating from the interface between cover glass and sample buffer. Knowing and controlling decay behavior and, consequently, the penetration depth (section 2.2.1) of the evanescent field is mandatory to ensure reproducibility of experimental results. In the following experiment a fluorescent bead $(r=50 \mathrm{~nm})$ was connected to the apex of an AFM cantilever (see below). This allowed to position the bead at a controlled distance from the surface. First, the cantilever was brought into contact with the surface. Then it was withdrawn in $10 \mathrm{~nm}$ steps. Every $10 \mathrm{~nm}$, the intensity was measured. The excitation intensity in the evanescent field is proportional to the detected bead emissions. Therefore, the detected intensity allows to quantify the evanescent field decay. The results are shown in fig. 4.9:

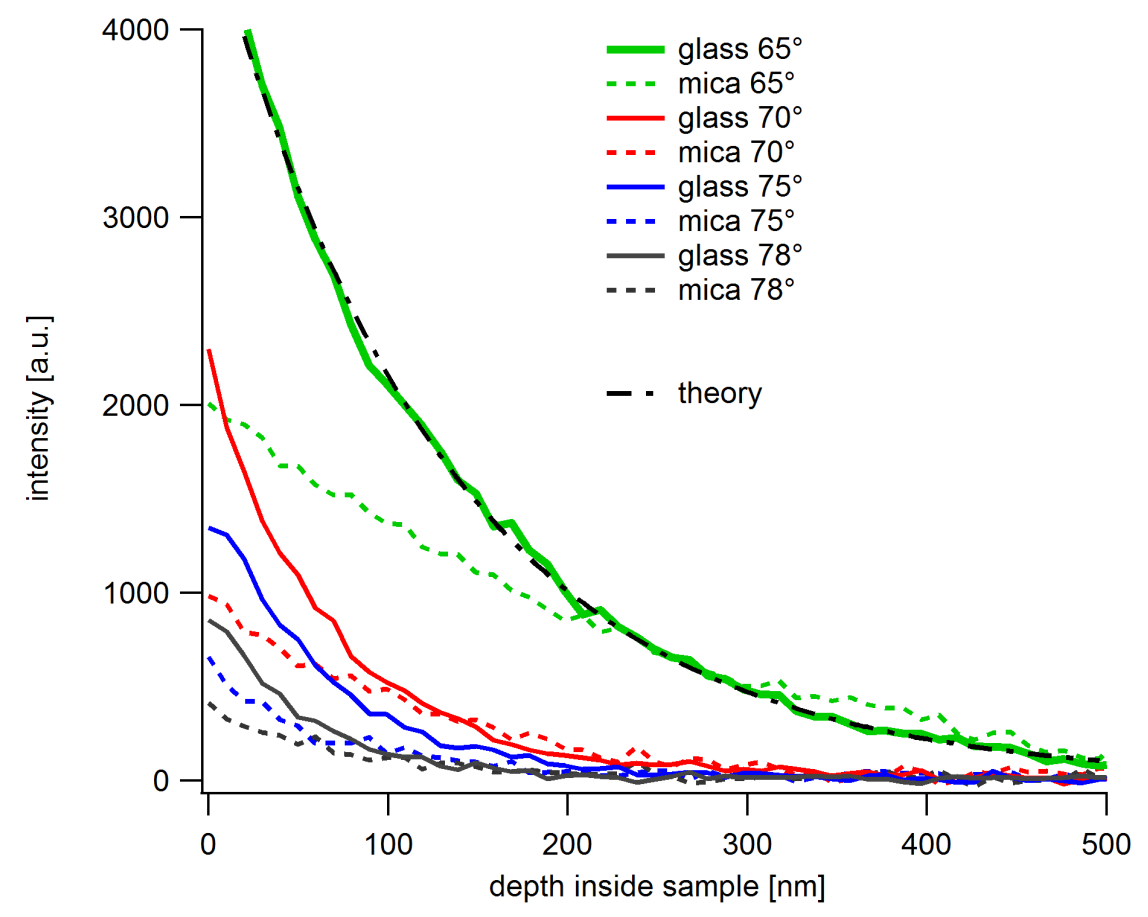

Figure 4.9: Measured evanescent field decay for different incidence angles on normal cover glasses and mica substrates.

For an incidence angle of $65^{\circ}$ on glass the measured evanescent field decay corresponds to the theoretical decay function 2.10. The bead dimensions (emissions from different depths) seem to cause no apparent distortions of the theoretically expected decay curve and the incidence angle dependent decay corresponds to the 
theoretical predicted penetration depths. The less pronounced decay on the mica substrate is probably a result of a changed angle on the way through the different layers of the substrate (section 3.6.3). Additionally, birefringence of the mica can play a role.

\section{Connecting the polystyrene bead to the cantilever tip}

By experimental variation of surface forces, conditions were found under which the bead could be picked up with the cantilever tip while imaging in contact mode. On plasma cleaned cover glass substrates $(2 \mathrm{~min}, 40 \mathrm{kHz}, 30 \mathrm{~W})$ the beads got stuck to the tip when adding $5 \mathrm{mM} \mathrm{MgCl}_{2}$ to the buffer (deionized water). The underlying mechanisms are probably similar to the one explained under section 3.6.3. On the Mica substrates $5 \mathrm{mM} \mathrm{MgCl}_{2}$ were needed to slightly immobilize the beads on the substrate, they got stuck to plasma cleaned tips $(30 \mathrm{~s}, 40 \mathrm{kHz}, 30 \mathrm{~W})$. Used cantilever: BL-RC150VB-HW, Olympus, Japan.

\subsubsection{Discussion}

During the design of the combined AFM-TIRFM setup special attention was given to prevent additional mechanical instrument noise. Since AFM is as mechanical technique highly sensitive to mechanical vibrations, the added TIRFM components have the potential to increase the noise level. Especially the camera and the image splitter can cause or amplify additional vibrations. The mechanical decoupling between the central microscope part and the image splitter / camera part enables high resolution imaging on the Actin interacting protein DBN-1 (section 4.5.2) and improved position control during quenching experiments with fluorescent labeled ribosomes (section 4.2.5). However, reducing the noise by mechanical decoupling goes along with a reduced fluorescence localization capability. Another demand on the setup was the possibility to change the penetration depth of the evanescent excitation field used in TIRFM. This allows to control the disturbing light background originating from AFM tips (section 4.2.4). Controlling the field depth allows to perform the ribosome quenching experiment (section 4.2.5) and the Adenovirus unpacking experiment with real time observation of single fluorescent molecules (section 4.5.3). 


\subsection{Single molecule fluorescence with TIRFM-AFM}

\subsubsection{Introduction to the experiments}

A potential application of the presented custom made AFM-TIRFM setup is the simultaneous detection of fluorescently labeled ribosome factors and the previously reported height fluctuations on top of these ribosomes (section 2.4.3). The height fluctuations were speculated to be associated with conformational changes of the ribosome. Such changes could be correlated with the binding and unbinding of single factors (tRNAs and EFG) to the 70S ribosome.

To record fluorescently labeled and transiently bound tRNAs and EFG, fast detection rates are required. Pedersen [72] reports that peptide bonding formation occurs at roughly $50 \mathrm{~Hz}$. According to the Nyquist Shannon-sampling-theorem, sampling frequencies of around $100 \mathrm{~Hz}$ would be required for their fluorescence detection.

The detection rate is influenced by three correlated conditions: A minimum signal to noise level is necessary which scales with the chosen excitation intensity. The excitation intensity is limited due to the fast bleaching times of single dye molecules at high excitation intensity. To determine the initial values and settings for adequate observation results, fluorescence experiments with single labeled $70 \mathrm{~S}$ ribosomes were conducted. In a second step, the effects of an environment are investigated where AFM probe, fluorescence excitation, and emission come together on the scale determined by the ribosome size. Finally, the fluorescence of labeled ribosomes in contact with the AFM probe was investigated.

\subsubsection{Bleaching times}

The detection of single fluorophores might be compromised by photo bleaching. Photo bleaching refers to the reduction of fluorophore emission levels by photochemical destruction. A famous bleaching mechanism is addressed to transitions from an excited single state to an excited triplet state. The triplet state has longer lifetime and, therefore, a higher interaction probability that facilitates covalent modifications. Those destroy the fluorescence capability. One way to decrease bleaching is to reduce the excitation intensity. To study the bleaching rates, $70 \mathrm{~S}$ 
ribosome complexes labeled at the outside with a single Alexa 488 dye molecule were investigated ${ }^{i}$. Under constant excitation conditions the intensity decay $I(t)$ can be described by a first order rate constant $k_{b l}[73]$.

$$
\frac{d I(t)}{d t}=-k_{b l} \quad k_{b l}=\frac{1}{\tau_{b l}} \quad I(t)=I(0) e^{-k_{b l} t}
$$

Fig. 4.10 shows the detected intensity decays for $\sim 30$ single labeled ribosomes at certain laser excitation intensities $I_{\text {exc }}$. The TIRF incidence angle was always adjusted to $63^{\circ}$. The intensity decay (dotted lines) is fitted with the single exponential function (solid lines) from eq. 4.3 to derive the bleaching time $\left(\tau_{b l}\right)$ which is defined as the time where $I(t)$ drops to $\frac{I(0)}{e}$. The ribosomes were immobilized on silanized cover glasses and immersed in TAKM7 buffer (section 3.3.1).

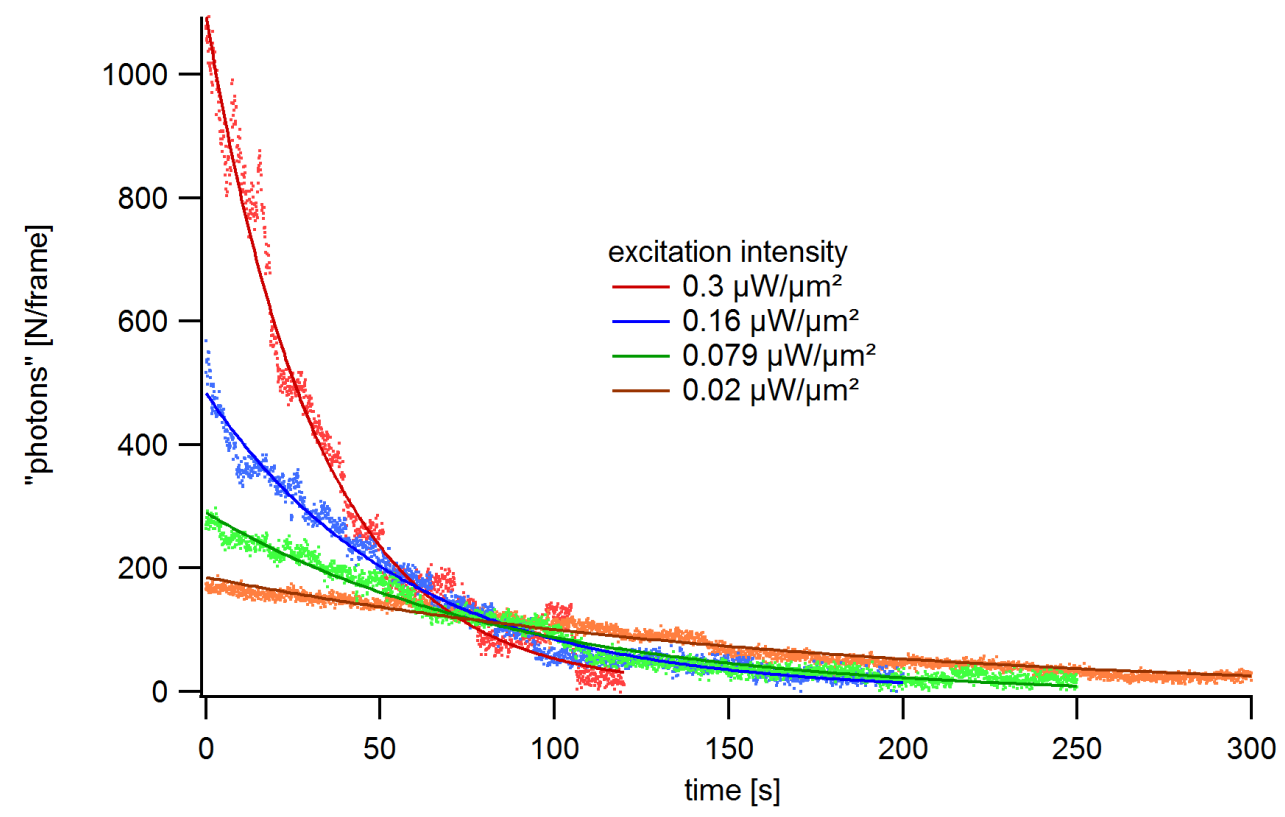

Figure 4.10: Intensity decay of $\sim 30$ single labeled ribosomes at varied laser excitation intensities.

In the detection path a bandpass filter with the center wavelength of $525 \mathrm{~nm}$ and a Full width at half maximum (FWHM) of $45-25 \mathrm{~nm}$ was used. The intensity was detected by an EMCCD camera. Exposure times were $100 \mathrm{~ms}$. The bleaching

${ }^{i}$ the labeld ribosomes were kindly provided by S. Adio and A. Konevega from the group of Prof. M. Rodnina at the MPI-BPC in Göttingen 
times for the chosen laser intensity are listed in tab. 4.1. At the lowest intensity a single labeled ribosome could be observed for $\sim 3 \mathrm{~min}$. At the highest intensity this was reduced to $\sim 30 s$.

Table 4.1: Bleaching times of single labeled ribosome samples at different excitation intensities.

\begin{tabular}{l|l|l|l|l}
$I_{e x c}\left[\mu W / \mu m^{2}\right]$ & $\mathbf{0 . 0 2}$ & $\mathbf{0 . 0 7 9}$ & $\mathbf{0 . 1 6}$ & $\mathbf{0 . 3}$ \\
\hline$\tau_{b l}[s]$ & 177 & 89 & 58 & 32 \\
\hline
\end{tabular}

Ribosomes with a single label at a more function related position are available in the form of a ribosome construct containing single labeled (Alexa 488) mRNA. For the investigation of these constructs and their fluorescence behavior, slightly higher excitation intensities were used. The intensity decays are shown in fig. 4.11.

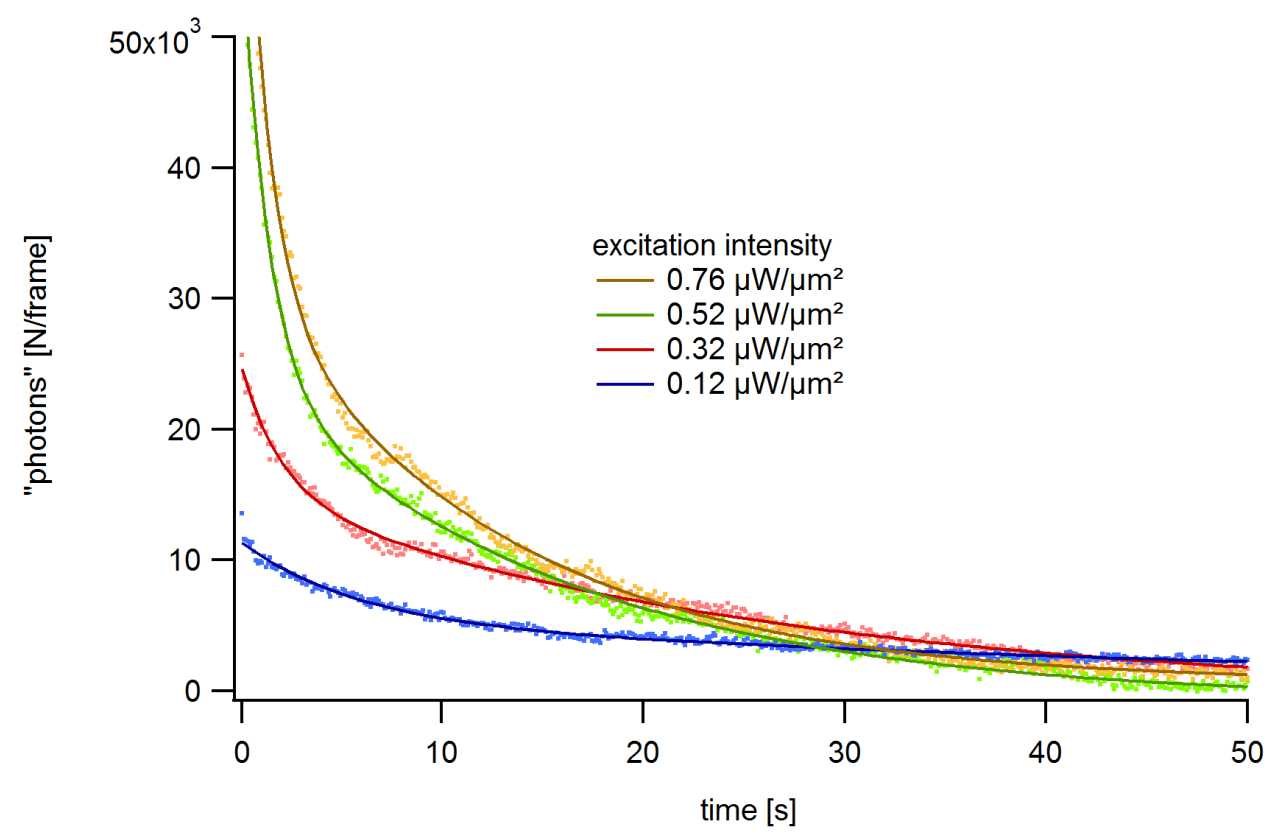

Figure 4.11: Intensity decay of $\sim 400 \mathrm{mRNA}$ labeled (single label) ribosomes at varied laser excitation intensities.

For these samples we found that a single exponential function returned a very poor fit to the intensity decays. The decay behavior of the mRNA bound labels seems to behave comparable to labels from the cyanine family bound to double-stranded DNA. For cyanine dyes (from which Alexa 488 is also synthesized) Kanony et al. [74] mention two processes to be responsible for bleaching. Those are addressed to different binding modes of the dye molecule. One mode is the external DNA 
binding (e.g. groove binding). Its fast bleaching is mediated by $\mathrm{O}_{2}$. The other is the intercalated dye molecule. Its slow bleaching is mediated by $\mathrm{OH}$ radicals. As a consequence, there are two rate constants in the bleaching process which can be determined by fitting a double exponential decay function:

$$
I(t)=I_{1} e^{\frac{-t}{\tau_{1}}}+I_{2} e^{\frac{-t}{\tau_{2}}}
$$

In our case the origin of the two rate constants is unclear, and might be related to alternative orientations of the dye. The bleaching times for the mRNA labels at the chosen laser intensity are listed in tab. 4.2. The faster rate constant is described by $\tau_{2}$ and the slower one by $\tau_{1}$.

Table 4.2: Bleaching times of mRNA labeled ribosomes vs. excitation intensity.

\begin{tabular}{l|l|l|l|l}
$I_{\text {exc }}\left[\mu W / \mu m^{2}\right]$ & $\mathbf{0 . 1 2}$ & $\mathbf{0 . 3 2}$ & $\mathbf{0 . 5 2}$ & $\mathbf{0 . 7 6}$ \\
\hline$\tau_{1}[s]$ & 75 & 26 & 16 & 13 \\
$\tau_{2}[s]$ & 20 & 6 & 1.3 & 1.2 \\
\hline
\end{tabular}

The bleaching times for the ribosome constructs labeled with the peripheral Alexa 488 label and the ribosomes constructed with the labeled mRNA are compared in fig. 4.12 . 


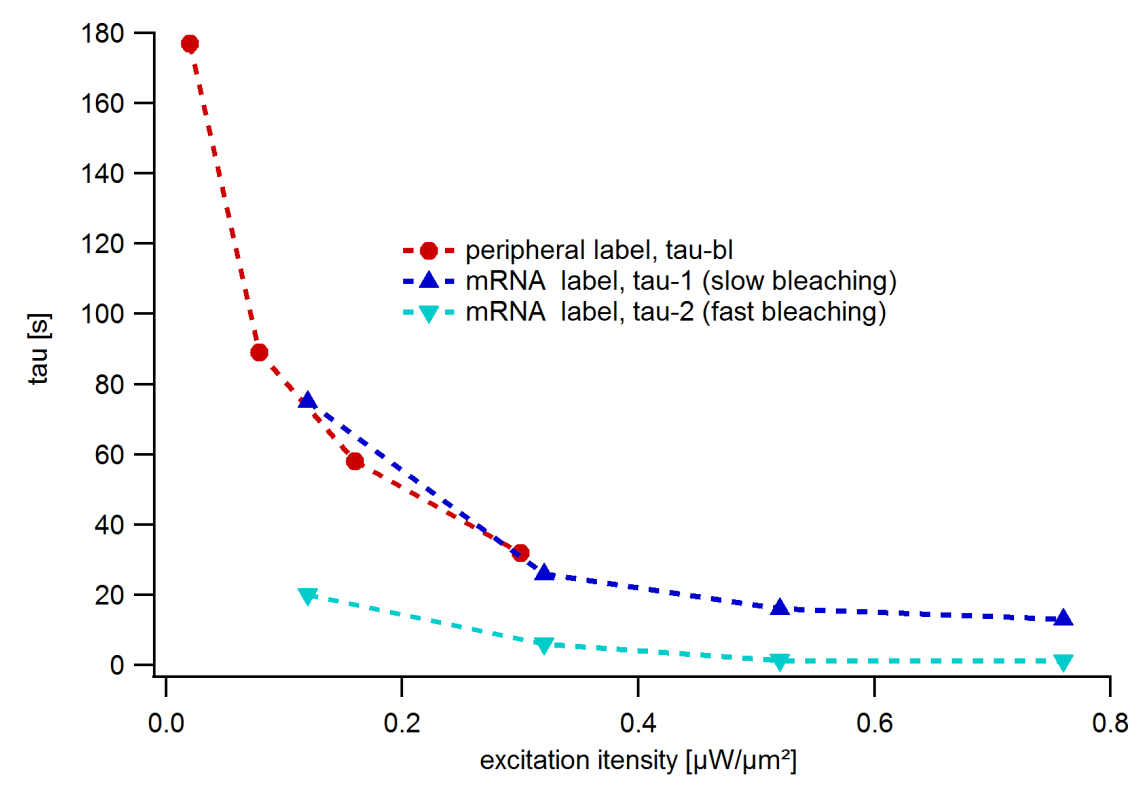

Figure 4.12: Bleaching times vs. excitation intensity for peripheral and mRNA labels. The bleaching time trend of the peripheral labels is followed by the mRNA labels which also show a second bleaching time constant indicating a second bleaching mechanism.

The trend for $\tau_{b l}$ is followed by $\tau_{1}$ for higher intensities so that the bleaching times of the peripheral labels seem to be comparable with the times for the mRNA labels. However, $\tau_{2}$ indicates a second, faster bleaching population of the mRNA labels.

To assign values to the signal heights of single dye labels under the presented bleaching conditions, respectively laser excitation intensity, a Fluorescence to Background Signal (FBS) is defined. The definition is determined by the mean of the emitted photons per frame $(100 \mathrm{~ms})$ during the on state $\bar{P}_{e m}$ (fig. 4.13a) minus its SEM, minus the background mean $\bar{B}$ plus its SEM after bleaching, $F B S=\left(\bar{P}_{e m}-S E M_{\bar{P}_{e m}}\right)-\left(\bar{B}+S E M_{\bar{B}}\right)$. Fig. 4.13b shows the FBS for the different excitation intensities. The error bars are the SEMs of the FBS means. 


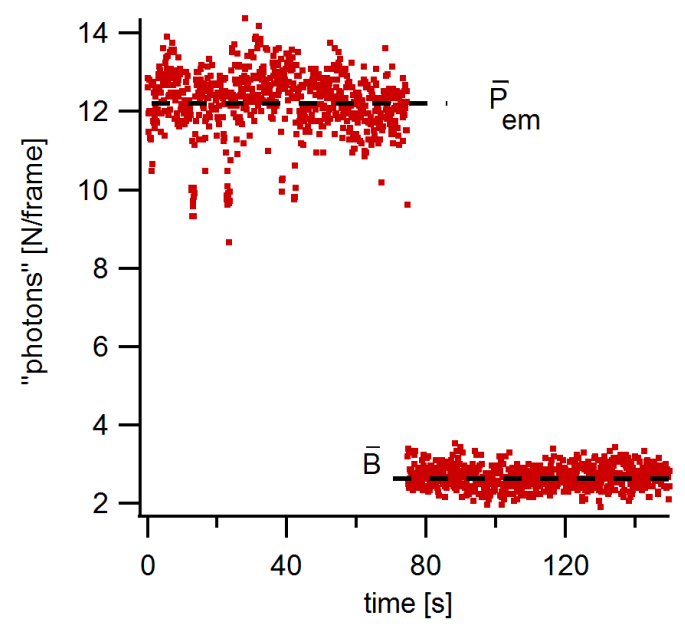

(a) FBS definition.

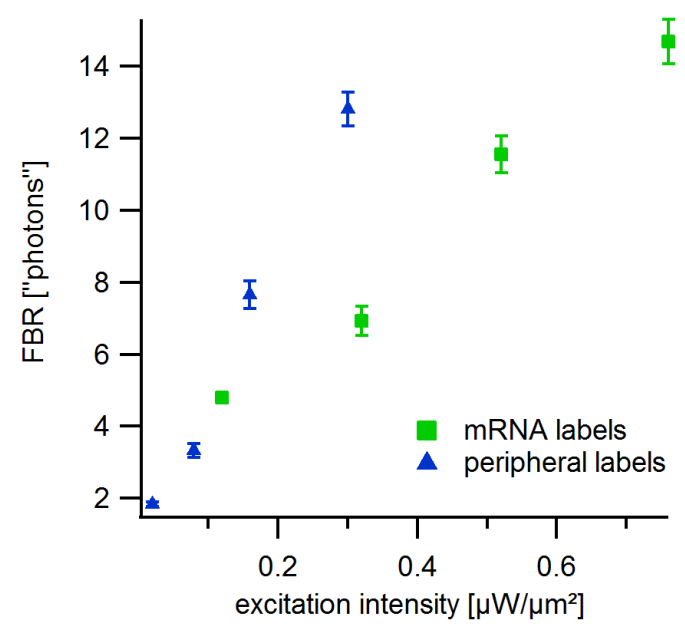

(b) $F B S$ vs. excitation intensity.

Figure 4.13: (a): The FBS definition. (b): The FBS scales approximately linearly with the excitation itensity. This allows to chose an excitation intensity for a designated FBS value.

Fig. $4.13 \mathrm{~b}$ reveals that even the lowest excitation intensity enables the detection of the appearance or disappearance of a single label. It allows to connect the $F B S$ value via the excitation intensity to the bleaching time in tab. $4.2 \& 4.1$.

\subsubsection{Achievable frame rates}

After evaluating the fluorescence signal of the labeled 70S ribosome constructs, a further question aris concerned maximal possible detection frequency. Again, the $F B S$ is used to estimate the expected signal change during an appearance or disappearance of a single label at different detection frequencies (fig. 4.14). As excitation intensity $0.76\left[\mu \mathrm{W} / \mu \mathrm{m}^{2}\right]$ was chosen. The Andor ixon3 860 EMCCD was used with an electron multiplying gain of $300 \%$ at a cooling temperature of $-80^{\circ} \mathrm{C}$. The quantum efficiency of the camera at the emission center wavelength $(520 \mathrm{~nm}$ ) is $\sim 96 \%$ (according to the manufacturer). 


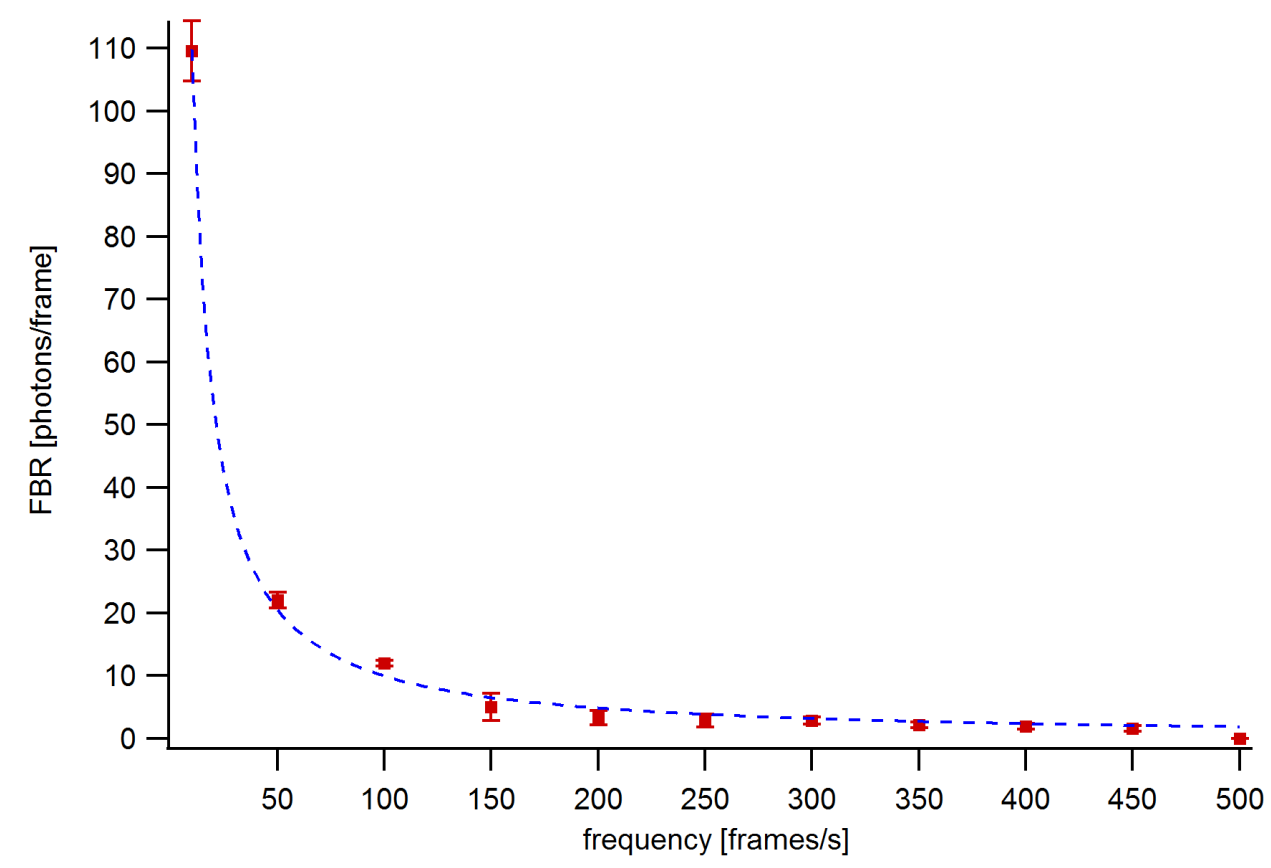

Figure 4.14: $F B R$ vs. detection frequency.

Since the $F B S$ is defined by the mean of photons per frame, the signal goes down with a time $e^{-1}$ behavior. In principle detection of single fluorophores up to 450 frames/s is possible, however, with an observation time of a few seconds due to the high excitation intensity. For most experiments a longer observation is preferred which can be achieved by using a lower excitation intensity, see tab. 4.2 \& 4.1 .

\subsubsection{Background light from AFM cantilevers during single molecule detection}

When placing the AFM tip into the TIRF excitation and emission volume, the background light originating from the cantilever and the tip apex can outshine emissions from single fluorophores even though appropriate band pass filters are used to block the excitation light.

Speculations about the nature of this additional AFM tip related light includes local field enhancement causing increased intensities of scattered excitation light and excitation light induced surface plasmons [12]. Efforts to measure the wavelength of the disturbing light with a normal sensitivity fiber coupled spectrometer 
(CCS100, Thorlabs, USA) did not work out. The light intensity was high enough to be detected with the EMCCD camera, but still too small for the available spectrometer. Another possibility to get information about the wavelengths would be to use several narrow band pass filters and to compare the detected intensities on the EMCCD camera.

The level of the background light compared to the emissions from single labeled ribosomes also depends on the illumination type, see fig. 4.15. In the following experiments the incidence angle of the excitation laser beam was changed from subcritical to super-critical incidence angles. In the sub-critical range the fluorophores were excited by brigthfield illumination and under super-critical angles by the evanescent field. In brigthfield mode the whole tip and cantilever are illuminated by the propagating laserbeam. In TIR mode there is no propagating wave but a shallow evanescent field, which illuminates just the apex of the tip (see section 2.2.1).

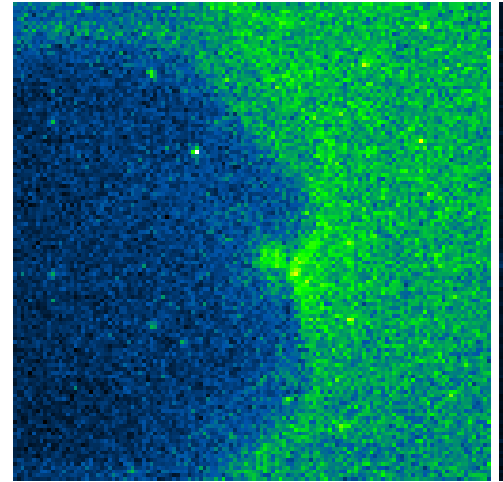

(a) laser brightfield

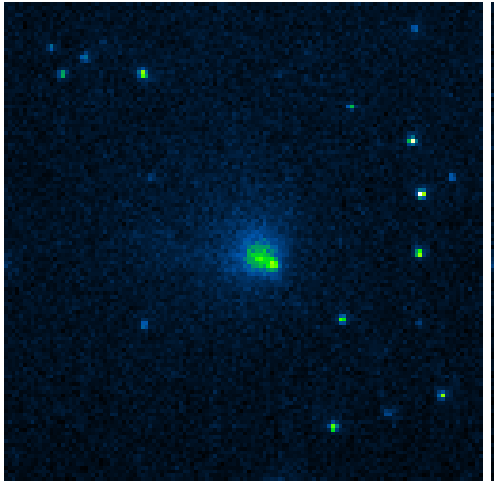

(b) close to critical angle

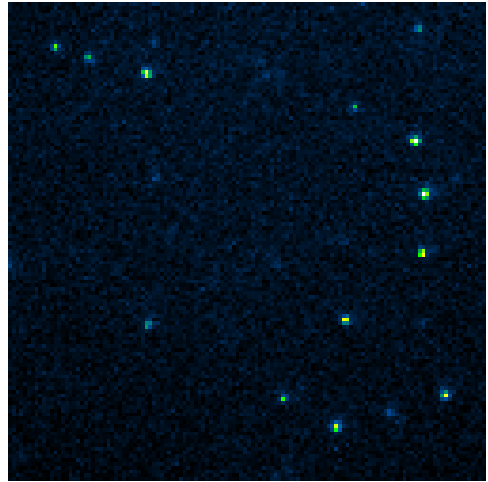

(c) TIRF

Figure 4.15: Cantilever-tip background and single labeled ribosomes vs. excitation mode. (a): laser incidence angle $10^{\circ}$. Only the cantilever outline is visible. (b): laser incidence angle $60^{\circ}$, close to the critical angle of TIR $\left(61^{\circ}\right)$. The ribosomes become recognizable but the tip apex is still visible. (c): laser incidence angle $65^{\circ}$, the tip apex is no longer visible.

Figures $4.16 \& 4.17$ show light emission from single labeled ribosomes (Alexa Fluor488 on mRNA) and the background signal from different AFM tips in contact with the substrate. The use of an Olympus BL150 cantilever with a Au coated, v shaped tip $(r=30 \mathrm{~nm})$ was compared with that of an Olympus RC800 cantilever with a Pyramidal shaped $S i_{3} N_{4}$ tip $(r=15 n m)$. Surface contact of the tips were ensured by a force set point of $200 p N$. The laser intensity was adjusted 
to $0.32 \mu \mathrm{W} / \mu \mathrm{m}^{2}$. Ribosomes were immobilized on silanized glass cover slips and immersed in TAKM7 buffer.

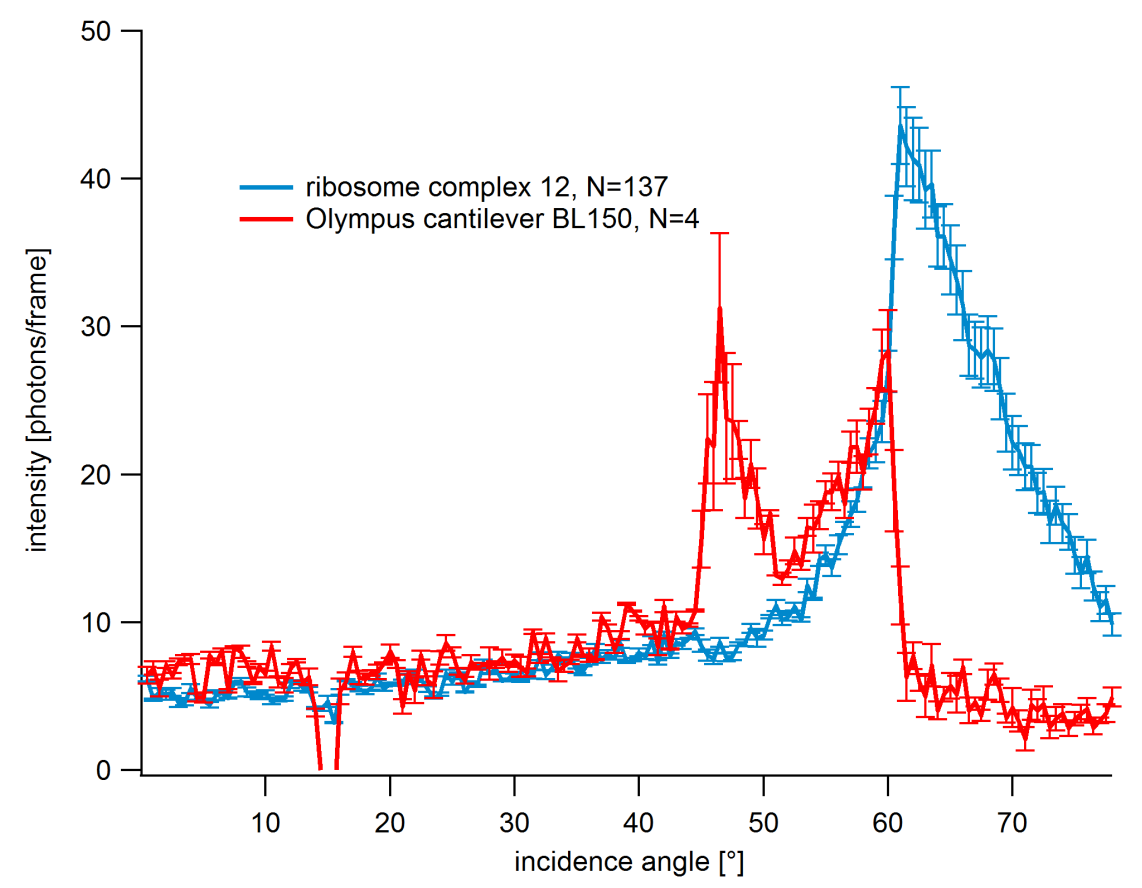

Figure 4.16: BL 150 cantilever vs. single labeled ribosomes from sub-critical to super-critical incidence angles. Means \pm SEMs.

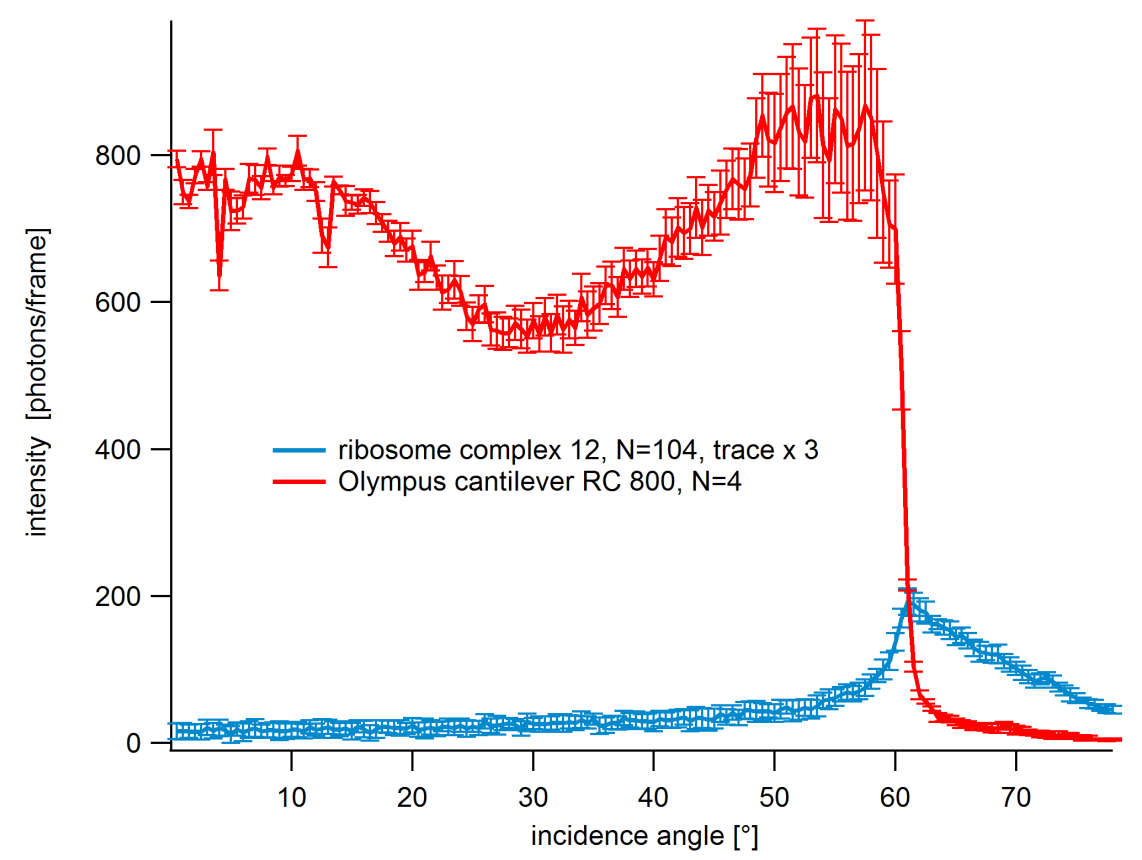

Figure 4.17: RC 800 cantilever vs. single labeled ribosomes from sub-critical to super-critical incidence angles. Means \pm SEMs. 
These results show that simultaneous AFM and single molecule fluorescence experiments are only possible in the TIRF illumination mode. The much higher background light originating from the RC800 cantilever is surprising since one of the enhancement effects is adressed to surface plasmons. This explanation, however, falls short in case of the $S i_{3} N_{4}$. The increase must be caused by a non-resonant field enhancement (section 2.2.2) or the pyramidal tip shape. The intensity processes follow the calulated angle dependencies with the typical intensity increase at the critical angle (section 2.2.2, fig. 2.6).

\subsubsection{Fluorescence quenching by AFM tips}

Picking up the contour height of single ribosomes with an AFM cantilever requires the tip apex to be in contact with the ribosome. Assuming the worst case, this means that the tip is also in contact with the dye molecule or at least separated by just a few nanometers. For separations of less than $5 \mathrm{~nm}$, the tip could quench the light emission of the single fluorophore completely [18]. In simultaneous AFM and TIRFM experiments with single labeled ribosomes we observed quenching with the gold coated Olympus BL150 cantilever in 3 out of 30 experiments (fig. 4.18).

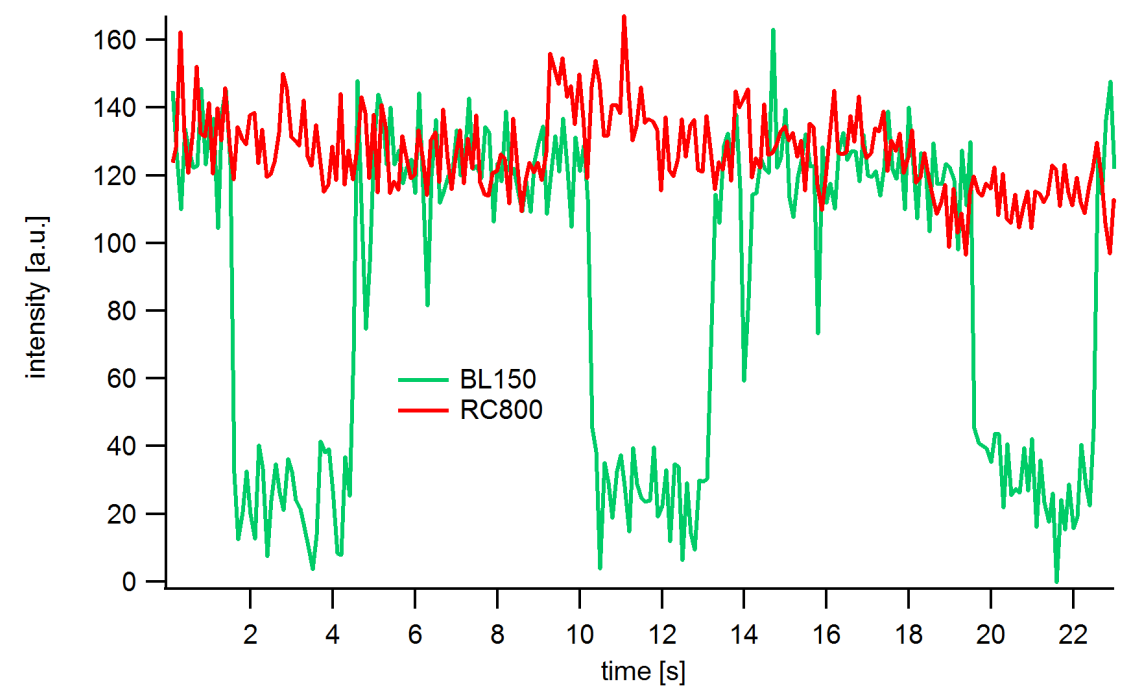

Figure 4.18: Quenching of a single labeled (at the outside) ribosome by a BL150 gold coated cantilever repeatedly brought into contact with a 3 second dwell. With the non-coated $\mathrm{Si}_{3} N_{4}$ RC800 tip quenching of fluorescence was never observed $(\mathrm{N}=30)$.

The distance between dye molecule and tip is hard to control since the immobi- 
lization of the ribosome on the silanized substrate is unspecific. A dye label which is located on the ribosome $(\varnothing \approx 20 \mathrm{~nm})$ periphery opposite to the tip will never be located in complete quenching distance. Furthermore, this might explain why we observed the quenching in only $10 \%$ of the experiments. In contrast, quenching was never observed with the Olympus RC800 $S i_{3} N_{4}$ cantilever. This is consistent with the literature [75] where less efficient quenching is reported with semiconducting tips.

\subsubsection{Disscussion}

The performed bleaching experiments deliver initial values for adequate excitation intensities and possible observation times. In experiments with transiently bound and fluorescently labeled ribosome factors it can be shown that an excitation intensity of $\approx 0.12 \mu \mathrm{W} / \mu \mathrm{m}^{2}$ will deliver a sufficient fluorescence signal. The estimated observation times should lie between $\approx 20 \mathrm{~s}$ and $\approx 75 \mathrm{~s}$ (tab. 4.2). Achievable frame rates can be as high as $400 \mathrm{~Hz}$ (fig. 4.14). However, they go along with increased excitation intensities and compromised bleaching times between $1.2 \mathrm{~s}$ and 13s. The background light from the cantilever tips is a limiting factor since it can outshine single fluorophores despite appropriate spectral filtering. The findings show that if tip and fluorophore are closer than roughly a micron, the fluorophore will only be detected in the TIRF illumination mode. The proximity of the AFM tip to the labeled structure creates another obstacle. It could be shown that the often used Olympus BL150 cantilever is able to quench the light emissions from single labeled ribosomes.

The quenching could also prevent the detection of labeled factors as envisaged in further experiments. The Olympus BL150 is one of the softest cantilevers available. With its high sensitivity and its low noise this cantilever is very often used for single molecule AFM experiments. Since the gold coating of the tip makes it an efficient quencher, measurements with a similar cantilever without a gold coated tip are of interest. The closest choice is the Olympus RC800 cantilever, for which we could not observe quenching. Quenching experiments with several AFM tips and separate single fluorophores could give further insight into methodological aspects, which is important for future research. 


\subsection{Practical AFM force set point limits in liquid environments}

Resolving contours and dynamics of soft macromolecular complexes with force probing techniques like AFM and optical tweezers requires to locate the soft surface by using the smallest possible contact force. Otherwise contours might be distorted and dynamics might be disturbed or even prevented. The challenge is to hold the contact force on its minimal level. This is done by using a force feedback. Such experiments are named force clamp experiments. In the extreme case, the feedback has to balance the interaction force with the sample and the influence of random thermal force by adjusting the probe strain. Gittes \& Schmidt [7] analyzed the minimal contact force to locate the "edge" of a force profile for an idealized feedback. A central finding is that force resolution and minimal contact forces are both limited by the thermal white noise force acting on the probe.

Practically, the mechanical cantilever noise is often judged by deriving the PSD of the thermal driven cantilever fluctuations and calculating the RMS force noise (equation 4.1) [70]. The RMS force noise (over the total frequency range) of a cantilever $20 \mathrm{~nm}$ away from surface is experimentally evaluated with $f_{r m s} \sim 19 \mathrm{pN}$ (fig. 4.22). This force seems to be suitable to be used as force set point. However, approaching a target with such low force set points rarely works on a practical level. During the approach the velocity dependent drag acts on the cantilever and the cantilevers drag coefficient increases close to the surface. Furthermore, an electrostatic mediated repulsion on the cantilever close to the surface might occur [76]. These effects add to the cantilever bending so that the force set point might be reached even before a real contact is achived. Moreover, the RMS expression does not include every possible fluctuation amplitude height. In terms of the peak to peak amplitudes, the RMS includes only $65 \%$ of the amplitudes presented in the signal. This happens since the RMS value is based on the standard deviation of the amplitude distribution. Consequently, 35\% of the peak to peak amplitudes are higher than the RMS value. Including $95 \%$ of the possible peak to peak amplitudes already reveals that amplitudes of $2 \cdot f_{r m s}$ can occur.

Interestingly, even force set points of $30 p N$ can be still problematic under certain conditions. In the presented examination, a force clamp experiment with a $40 \mathrm{~s}$ 
dwell time was performed. Silanized cover glasses with TAKM7 buffer were used (section 3.3.1). The experiment was conducted with a low feedback gain $(\mathrm{I}=0.01)$. Surprisingly, the resulting deflection vs. time signal looks like active height changes occur during the experiment (fig. 4.19).

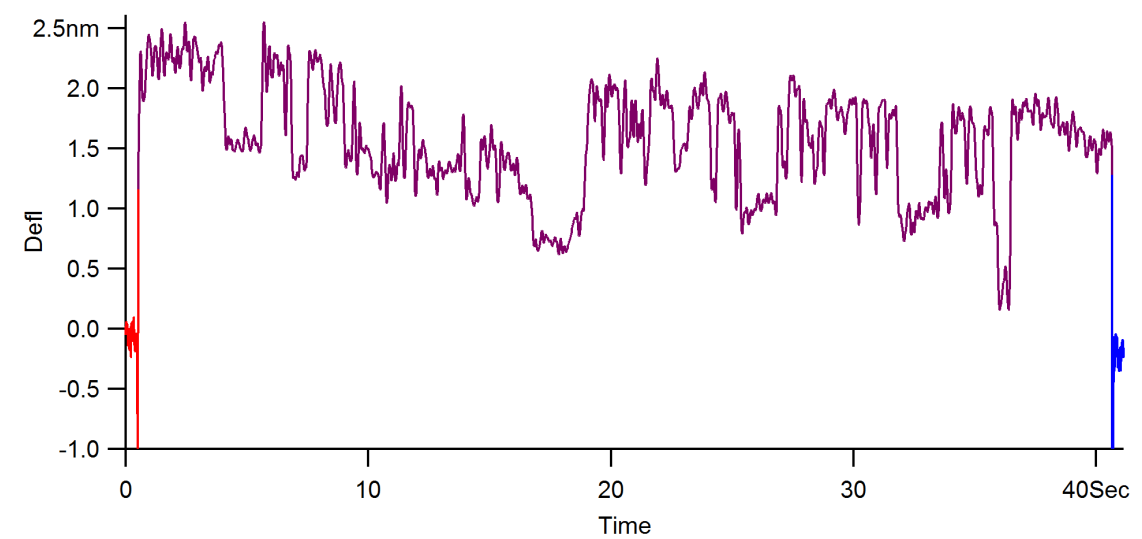

Figure 4.19: Artifacts in force-clamp experiments with a low force set point $(30 p N)$ and low integral gain. During the $40 s$ long dwell in the force-distance curves, jumps occur which look like active height changes of the underlying substrate. Cantilever: BL-RC150VB-HW, Olympus, Japan.

This kind of artifacts can be found in $3 \%(\mathrm{~N}=300)$ of the measurements described above. The height/depth of the 'jumps' ranges from $\sim 0.4 \mathrm{~nm}$ to $\sim 1 \mathrm{~nm}$. To deform a cantilever by these distances requires forces between $\sim 11 p N$ and $\sim 27 p N$ $(f=\kappa z)$. As one possible interpretation of these fluctuation the tip jumps in and out of contact. A jump into contact can be based on the following mechanisms: Close to the surface the electrostatic mediated repulsion acts on the tip. At very small separations, however, attractive van der Waals forces come into play and the tip jumps into contact [77], [78]. Müller et al. [76] reports jump distances from $\sim 0.51 \mathrm{~nm}$ to $\sim 0.61 \mathrm{~nm}$ in experiments on purple membrane in physiological buffer with blunt tips. To explain the jump out of contact, one can think of a force which pulls the cantilever tip out of adhesive contact. The main suspect for creation of such a force here is sample drift. In fig. 4.20 the results of a drift investigation during force-distance curves with a $40 s$ dwell are plotted. 


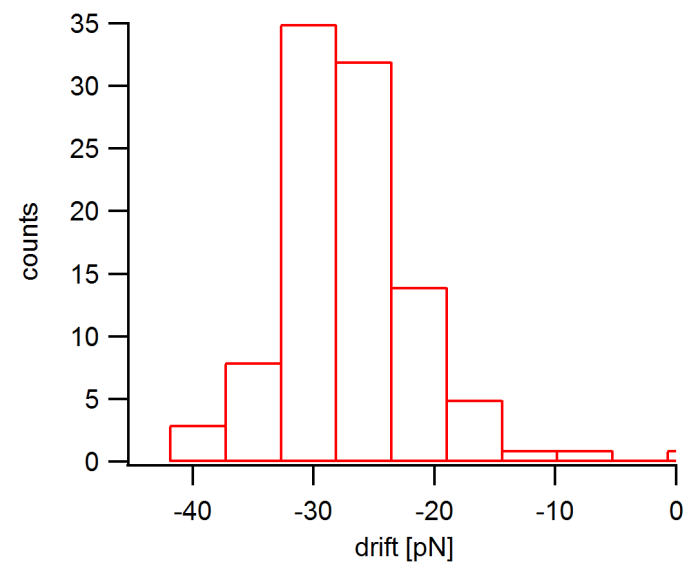

Figure 4.20: Drift during force-distance curves with a $40 s$ dwell on the surface $(\mathrm{N}=98)$. The result is expressed as a force signal. The drift is attributed to a slow separation of the sample holder from the AFM head. Same experimental conditions as in fig. 4.19 .

The results reveal existing drift forces which lead to a cantilever bending of around $\sim 0.9 \mathrm{~nm}$. To proof which forces are necessary for a jump out of contact, adhesion forces experiments were performed. The adhesion force maps cover a square area of $200 \mathrm{~nm}$ by $200 \mathrm{~nm}$ with 10 by 10 force-distance curves. The results are shown in fig. 4.21a.

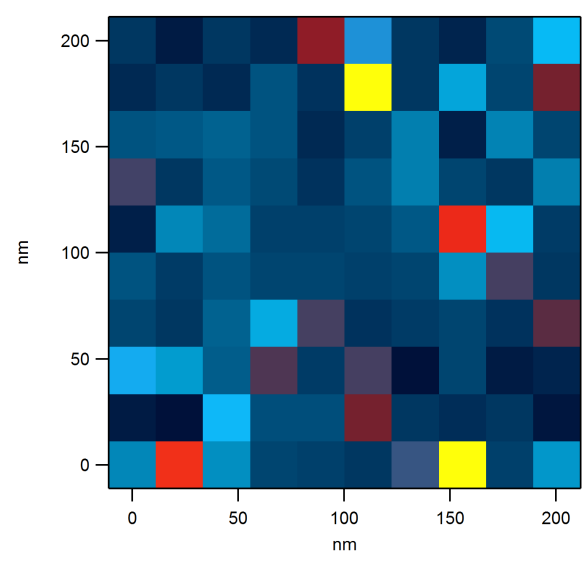

(a) Adhesion map.

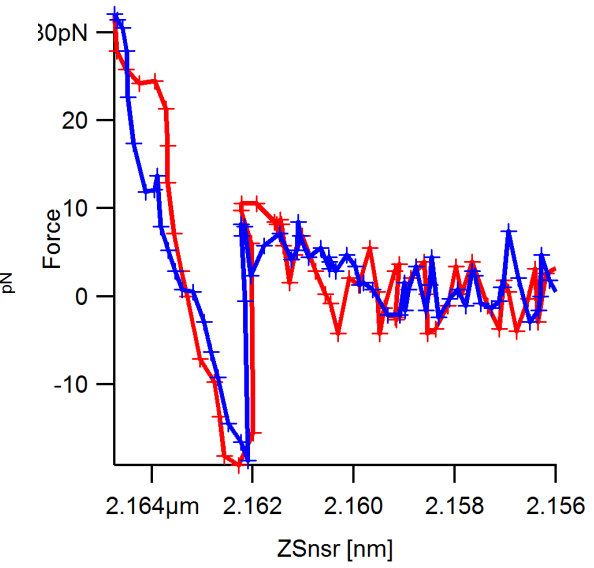

(b) Jump in and out of contact.

Figure 4.21: Adhesion forces and their influence on the cantilever. (a) The adhesion force experiments show adhesion forces which would bend the cantilevers before contact loss with values corresponding to the jump heights in fig. 4.19. (b) A single force-distance curve from the adhesion map. The force jumps during the approach (red) and withdraw (blue) correspond to deflections of $0.8 \mathrm{~nm}$ and $0.9 \mathrm{~nm}$. Same cantilever, surface, buffer as in fig. 4.19. 
These maps are screened for forces-distance curves where approach (jump in contact) and withdraw (jump out of contact) have jump amplitudes between $\sim 11 p N$ $(\sim 0.4 n m)$ and $\sim 27 p N(\sim 1 \mathrm{~nm})$. The analysis reveals that $14 \%(\mathrm{~N}=100)$ of the force distance curves fulfill the criteria. Fig. 4.21b shows such a force distance curve. To construe the fluctuations in fig. 4.19, it has to be explained how the cantilever jumps back into contact. Once jumped out of contact, the separation between cantilever and substrate is only very small. Since the cantilever fluctuates due to its thermal noise response, it may come so close to the surface that it jumps back into contact. Fig. 4.22 shows the cantilevers positional noise in z direction.

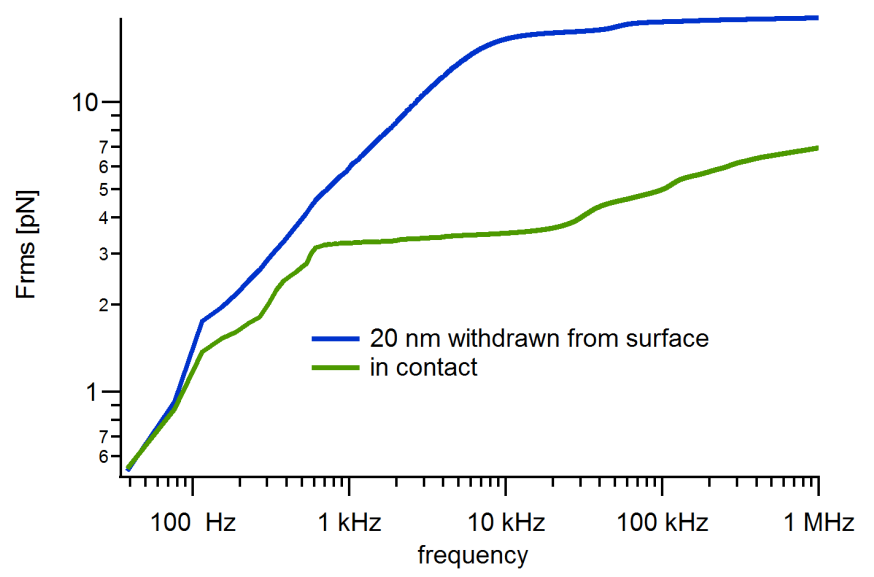

Figure 4.22: RMS force noise for different separation of cantilever, calculated with equation 4.1. Same cantilever, cover glass, buffer as in fig. 4.19 .

The RMS cantilever force noise of $\sim 19 p N$ (surface separations of 20nm) can be transferred to a fluctuation of $\sim 0.6 \mathrm{~nm}$. This shows, the possibility might exist that the tip jumps back into contact with the surface. If these mechanisms keep running, the resulting position signal will show reversal height jumps.

In conclusion, the presented experiments show that care should be taken when force clamp experiments are performed at forces close to $f_{r m s}$. Since an observed height step cannot be unambiguously attributed to motions of the sample.

\subsection{The optical tweezers setup}

This section is in part reproduced from Bodensiek et. al, RSI 2013 [58]. 


\subsubsection{Opto-mechanical design}

The majority of all optical trapping experiments are performed in a plane lying parallel to the microscope coverslip. In this horizontal geometry it is difficult to compare the quantitative results of optical trapping experiments with AFM indentation experiments because the experimental boundary conditions are very different. The ability to operate the trap in a direction perpendicular to the surface [61] and to detect the axial position of the bead in the trap [79] has been demonstrated before. This section describes the development of the optical trap in detail. The optical trap allows stable force spectroscopy in the vertical direction so that it can be applied routinely to investigate the response of biomaterials and macromolecules at single $p N$ forces. Specific attention is given to the following points:

- Integration of a stable optical trap into an upright optical microscope.

- Obtaining nearly constant trap stiffness over a vertical length of $100 \mu m$ by the use of a high NA water immersion objective.

- A low-loss optical design to obtain a relative high maximum trapping force of $80 p N$ with a $300 m W$ single mode LD.

- A short optical path to maximize the instrumental stability and to achieve $\mathrm{nm}$ and sub- $p N$ accuracy.

- The effect of reflections between the bead and the coverslip that create a micrometer sized interferometer.

- A fast field programmable gate array (FPGA) based force feedback loop that allows a millisecond response time.

For the assembly of the vertical optical trap each element was carefully chosen to ensure maximal long-term stability and a minimal sensitivity to vibrations and drift. A commercial upright microscope body (Eclipse 50i, Nikon, Japan) was used as platform since it provides both the focusing mechanism and the $\mathrm{x}-\mathrm{y}$ stage in a compact and stable castaluminium body. The additional optics to couple the near infrared laser beam into the optical path of the microscope were mounted on a custom-made optical table that was placed on top of the microscope (fig. 4.23). 


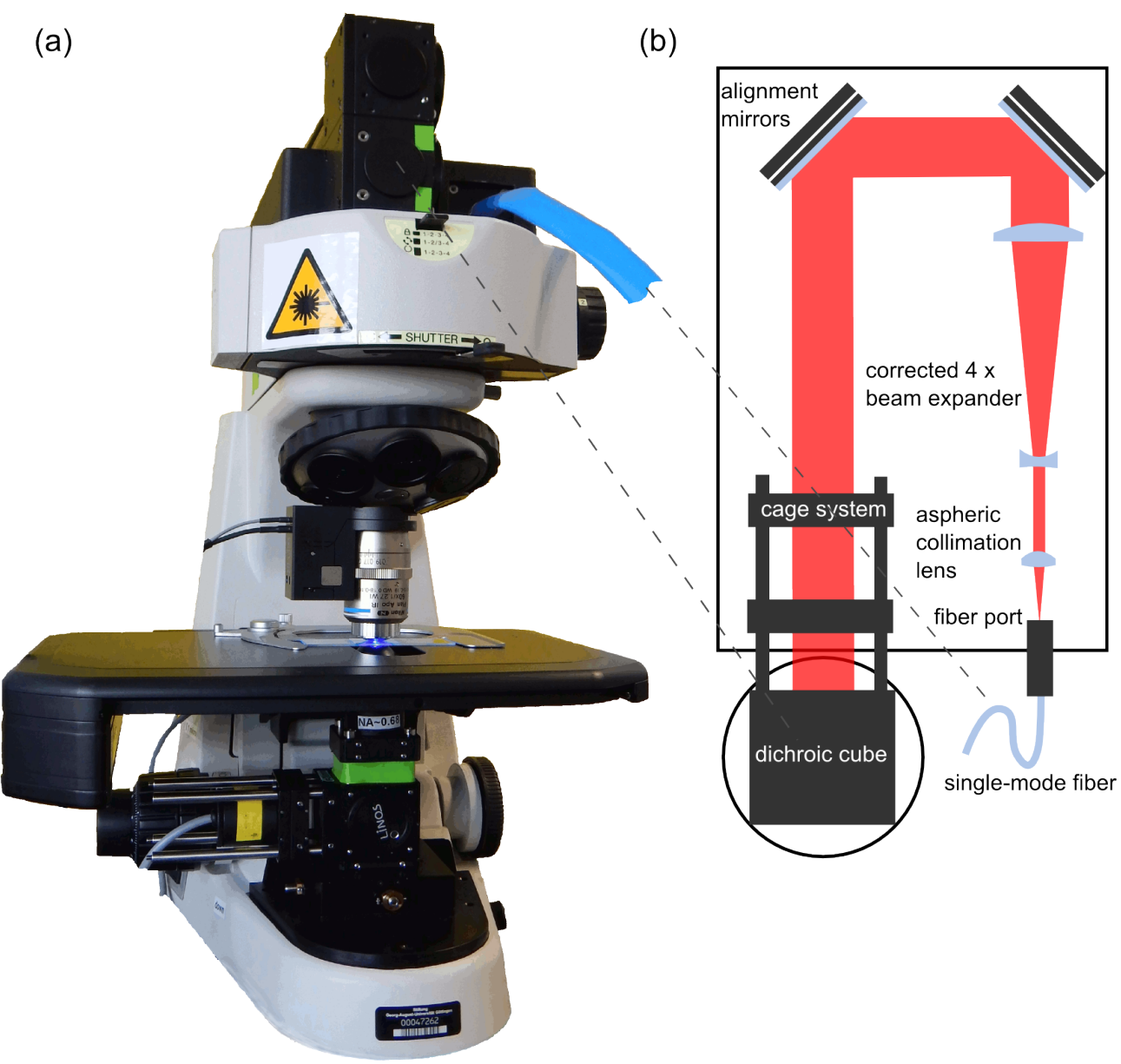

Figure 4.23: Mechanical design of the vertical optical trap. (a) A photograph of the complete instrument. (b) The additional optics to form the optical trap were mounted on a small optical table that was rigidly connected to the top-part of the microscope. The position detector and the Kohler illumination were mounted under the standard condenser. Other modifications to the microscope were limited to an objective-piezo and a motorized xy stage. The power supply for the LED and the analogue electronics to compute the bead position from the QPD signal were mounted on the back of the microscope.

Instead of mounting the laser optics on a separate optical table, the advantage of the approach used here is the increased mechanical rigidity of the whole system. Furthermore, the optical path of the laser beam can be kept as short as possible $(65 \mathrm{~cm})$. Due to this compact design, all optics are easily enclosed which prevents disturbing air flow in the beam path. These measures increase the resistance of 
the system against mechanical vibrations and ensure the safety of the operator. The detailed design and principles of the optical beam path in the instrument are explained under method section 3.4.1.

\subsubsection{Force limits}

For each trapped bead we determined the response of the detector (in $m / V$ ) and the trap stiffness (in $N / m$ ) by recording the thermal motion of the bead in the trap. The motion was converted into a power spectrum, which was then fitted with equation 2.18 in section 2.3.2. Using the maximum $300 \mathrm{~mW}$ output power of the optical fiber and $0.76 \mu \mathrm{m}$ diameter polystyrene beads, we typically obtained a trap stiffness of $0.2 p N / n m$ along the vertical axis. Without modifications to the optical system, beads made out of different materials and with different diameters can be used (we successfully tested both silica and polystyrene beads from 0.5 to $1.5 \mu \mathrm{m})$.

For experiments that require a lower trap stiffness, the laser power can be reduced since the trap stiffness scales almost linearly with the laser power. To achieve an optimal focus quality, it is critical to adjust the objective correction collar to the cover slip thickness. Figure 4.24 shows that the adjustment of the correction collar also has a large effect on the trap stiffness. We performed this optimization for each coverslip that was mounted by trapping a bead at a distance of $\approx 10 \mu \mathrm{m}$ from the coverslip and adjusting the correction collar until a maximum stiffness was obtained. 


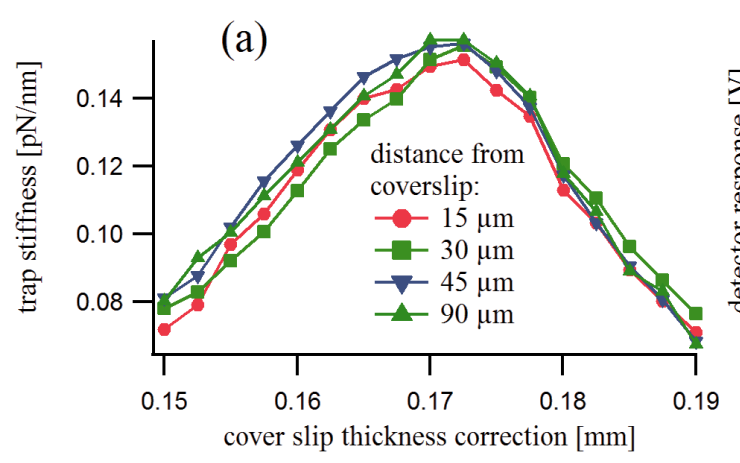

(b)
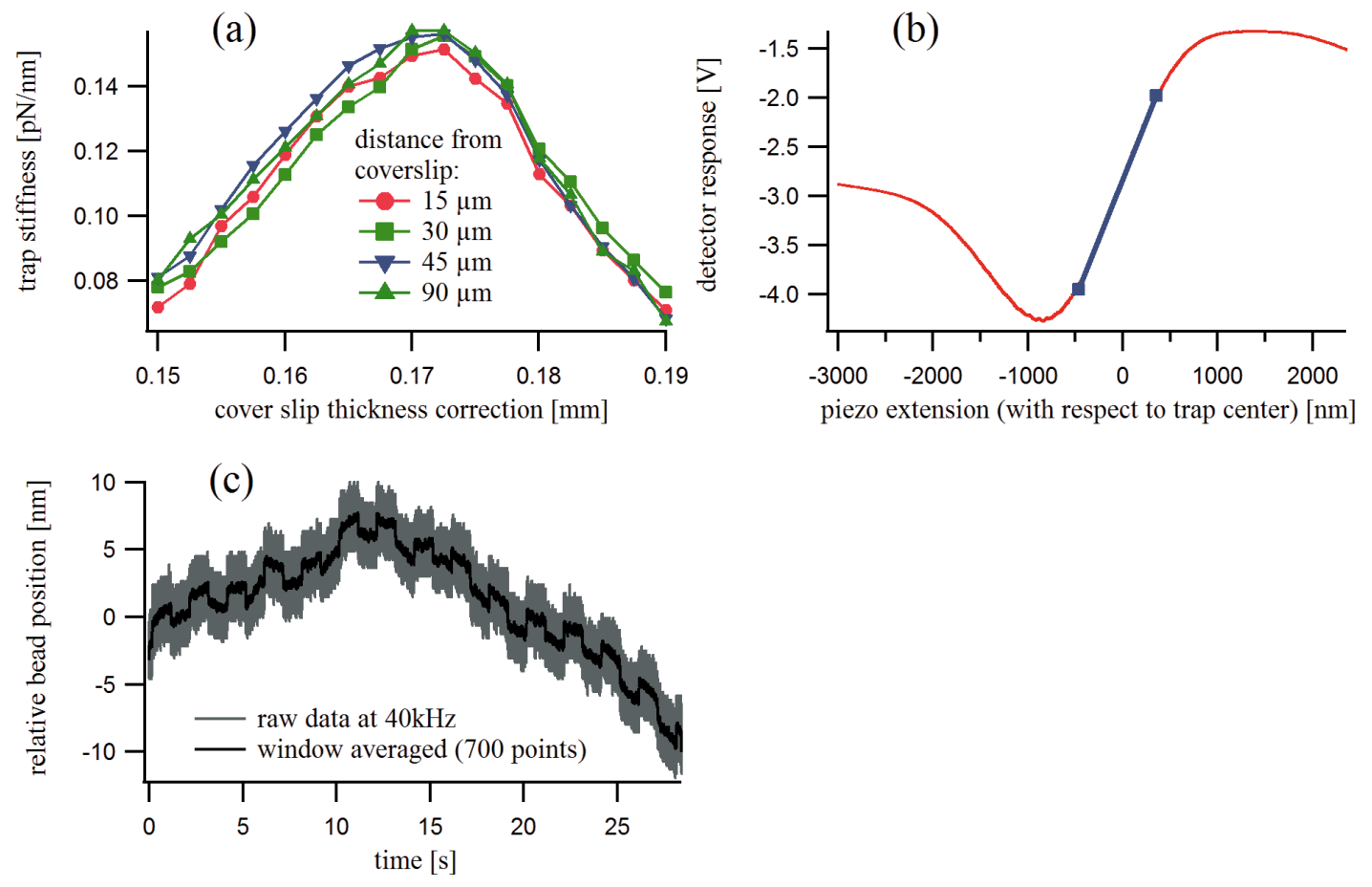

Figure 4.24: Trapping and detection performance. (a) Adjustment of the "coverslip thickness correction" collar of the objective is critical to achieve the maximum trap stiffness. At every distance from the coverslip the highest trap stiffness was obtained by adjusting the correction collar for each new sample chamber. (b) To measure the linear range of the vertical optical trap, we focused the trap onto a $760 \mathrm{~nm}$ diameter bead that was fixated in a $0.5 \%$ agarose gel. The trap was swept through the stuck particle in the z-direction. The detector signal shows that the response is linear for $\approx 0.8 \mu \mathrm{m}$. From the slope the detector sensitivity can be obtained $(0.43 \mu \mathrm{m} / \mathrm{V})$. However, for most experiments the thermal noise method described in section 2.3.2 was used. (c) To probe the resolution of the detection, we trapped a $760 \mathrm{~nm}$ diameter bead and moved it towards the glass coverslip where it attaches. Then, the trap was moved up-and-down by $2 \mathrm{~nm}$ steps using the objective z-piezo stage. For this specific experiment, we used a passive three-times voltage divider to increase the bit-depth of the FPGA output signal for such small signals. In the unfiltered as well as in the the window averaged data the steps are clearly visible.

The exertable force is given by the product of the trap stiffness and the displacement of the bead out of the trap center. To quantify the linear range of the trap, a $760 \mathrm{~nm}$ diameter bead was placed into a $0.5 \%$ agarose gel and the trap center was moved up-and-down through the bead. For displacements of up to $0.4 \mu \mathrm{m}$ away from the trap center, the signal is linearly proportional to the displacement (Figure 4.24(b)). Combined with a trap stiffness of $0.2 p N / n m$, a maximum force of $80 p N$ could be reliably detected. To test the limits of the vertical detection sensitivity, the experiment was repeated with moving the trap in steps of $2 \mathrm{~nm}$. 
The detector response (Figure 4.24(c)) shows that such small displacements are still clearly detectable. By multiplying the $2 \mathrm{~nm}$ steps by the $0.2 \mathrm{pN} / \mathrm{nm}$ trap stiffness, a force resolution of $0.4 p N$ results. We believe, however, that $2 \mathrm{~nm}$ represents an upper limit for the displacement resolution. The noise of the piezo controller, the mechanical vibrations of the microscope coverslip, and the linkage between the bead and the coverslip all contribute to the observed noise. The noise might partially mask the ability to detect smaller displacements. The slow fluctuation of the signal in Figure 4.24(c) is caused by a thermal drift of the stage in the z-direction with respect to the trap center which is typically limited to $1 \mathrm{~nm} / \mathrm{s}$.

To quantify the intrinsic noise limits of the vertical optical tweezers instrument, we recorded the fluctuations in the bead position and compared it to the fluctuation of a low-noise AFM cantilever in water. Figure 4.25a shows the force noise in form of the PSD. The RMS force noise is calculated with equation 4.1. In general, the total amount of noise is lower for the optical tweezers. From figure $4.25 \mathrm{~b}$ it can be seen that the total RMS force noise value of the optical tweezers is with $0.2 p N$ much lower than for the AFM cantilever $20 p N$.

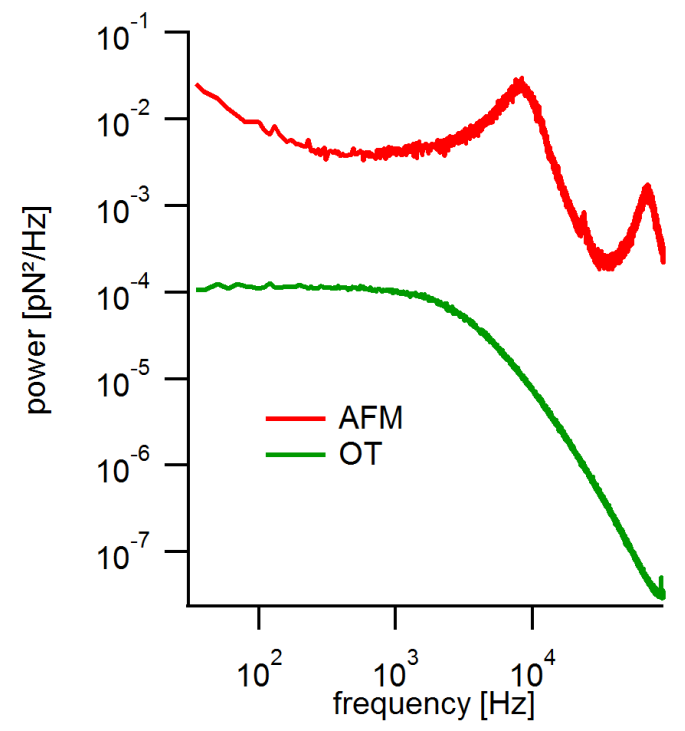

(a) PSD

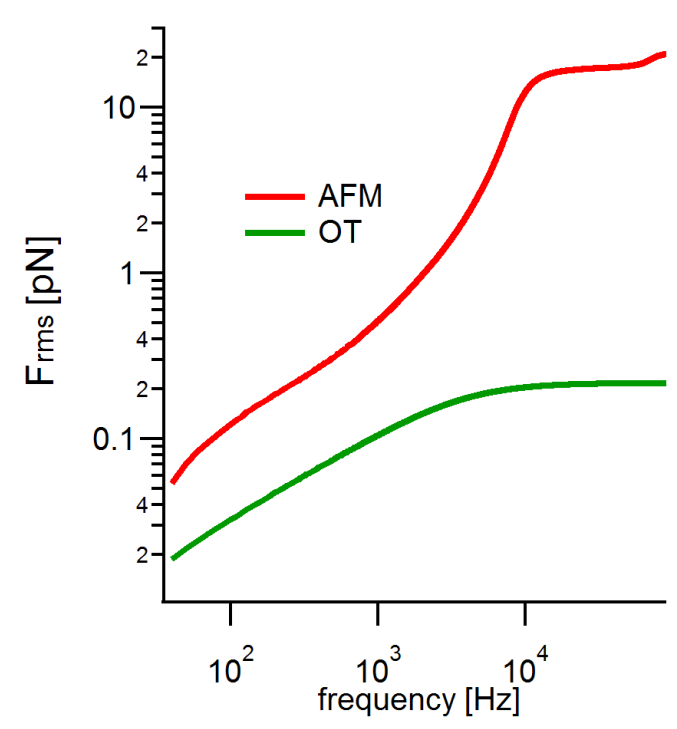

(b) Force noise

Figure 4.25: The intrinsic force noise in AFM and optical trapping. AFM cantilever in red $(\mathrm{BL}-\mathrm{RC} 150 \mathrm{VB} \kappa=0.03 \mathrm{~N} / \mathrm{m}, 30 \times 60 \times 15 \mu \mathrm{m})$. Trapped micro sphere in green $(r=380 \mathrm{~nm}$, trap stiffness $0.2 p N / n m)$

Figure 4.25a shows the characteristic peak for the AFM cantilever at the fundamental resonance frequency around $8 k H z$. The trapped bead behaves like an 
over-damped oscillator and shows a plateau before the spectrum falls off at about $2 k H z$, the resonance frequency of the trapped particle.

From the presented results we suggest, that performing low force spectroscopy and measuring macro-molecule dynamics with an optical tweezers instrument is the method of choice. However, the advantage of AFM is that it can also be used to image the sample. It is a useful feature to localize the sample before the mechanical measurement.

\subsubsection{Trap stiffness in vertical direction}

Since biological samples such as cells can reach heights of more than $10 \mu \mathrm{m}$ and many of the deformation experiments are performed within a range of multiple $\mu m$, it is essential that the performance of the optical trap remains constant away from the cover slip and over a large z-range. This requirement excludes the use of an oil immersion objective as it suffers from increasing spherical aberrations when the focus is moved away from the coverslip. This results in a decrease of the trap stiffness away from the coverslip [80] and a focus shift (the actual focus in the sample moves less than the objective displacement) [20]. Both are due to the refractive index mismatch between the immersion oil (1.51) and aqueous sample media (1.33). For optical traps the focus shift has been estimated to be 0.82 (ratio of focus to objective motion) [81], which would complicate the analysis of the experiments that are performed in the vertical direction. Both limitations can be overcome by the use of a water immersion objective, which delivers a constant quality of the focus and is, therefore, expected to deliver a constant trap stiffness throughout the sample [81]. To test this, a bead was trapped in a sample chamber, consisting of a coverslip and an opposing microscope slide approximately $100 \mu \mathrm{m}$ away. We moved the trapped bead in vertical direction from one end of the sample chamber to the other and calculated the trap stiffness at steps of $0.5 \mu \mathrm{m}$. Figure 4.26 shows that throughout the experiment the trap stiffness remained nearly constant and only decreased when the bead was within $\approx 10 \mu \mathrm{m}$ distance away from a surface. This observed reduction in the apparent spring constant can be largely explained by the increase of the hydrodynamic drag on the particle close to the surface [20]. When the increased drag was included into the calculation of the trap stiffness, the obtained value was linear within $5 \%$ of the mean. 


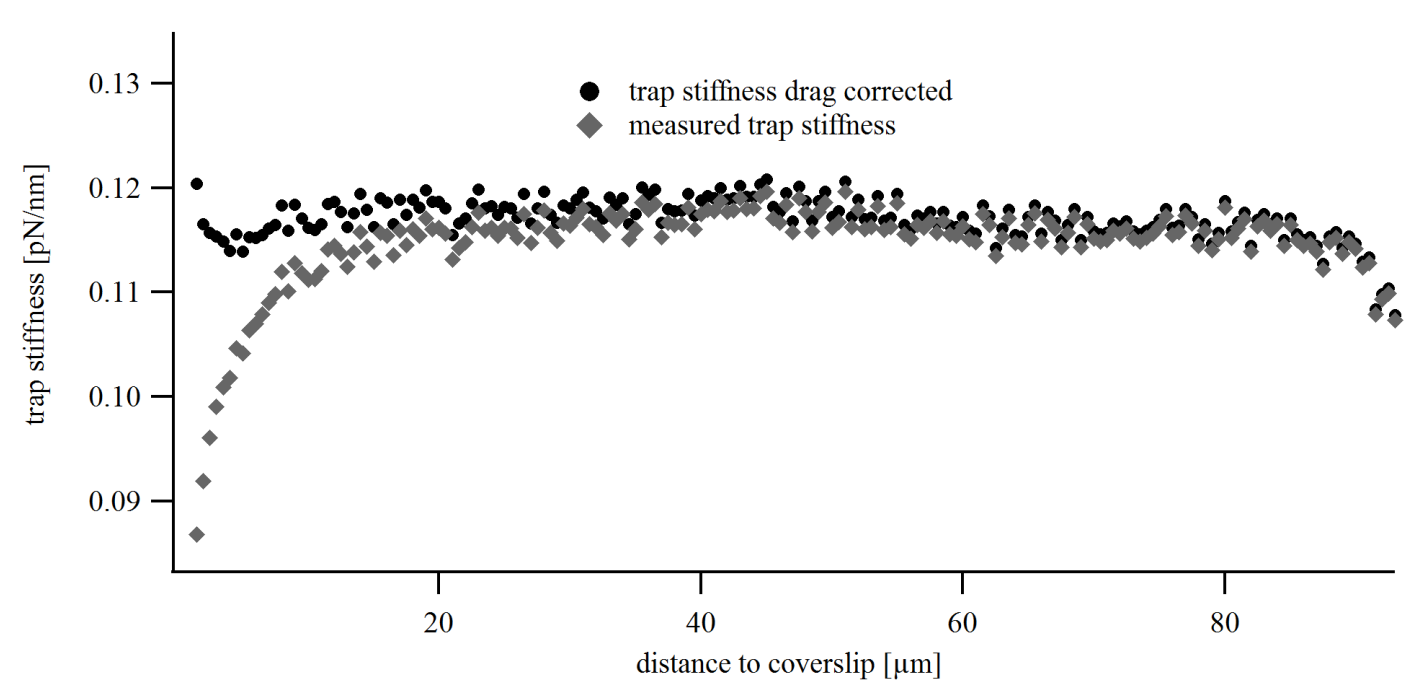

Figure 4.26: Linearity of the trap stiffness in vertical direction. Gray, the trap stiffness as a function of the distance to the coverslip is calculated using Eq. 2.18. A constant drag coefficient acting on the bead is assumed. Black, the increase in drag due to the vicinity of the coverslip is included in the calculation using Eq. 2.21. The spring constant of the trap is nearly constant throughout the whole sample over a length of almost $100 \mu \mathrm{m}$. The decay at $90 \mu \mathrm{m}$ is caused by the increase of drag due to the presence of the microscope slide on the other side of the sample cell which is not corrected for.

\subsubsection{Interferometry between the trapped bead and the surface}

As a consequence of operating an optical trap in the vertical direction, a part of the laser light will be scattered by the bead and "reflects" between the bead and the coverslip. The reflected light will interfere with the non-diffracted light. A total intensity on the QPD results that varies with the distance between the bead and the coverslip. This effect is called Fabry-Perot interferometric effect and visualized in Figure 4.27(a). The periodicity $\left(\lambda_{\text {measured }}\right)$ depends on the center wavelength of the laser $\left(\lambda_{\text {laser }}=974 n m\right)$ and the refractive index $(n)$ of the medium: $\lambda_{\text {measured }}=$ $\lambda_{\text {laser }} / 2 n$. This interference effect results in an artificial displacement (and force) signal that is as high as $10 \mathrm{~nm}$ when the operation takes place very close to the surface (Figure 4.27(a)). 

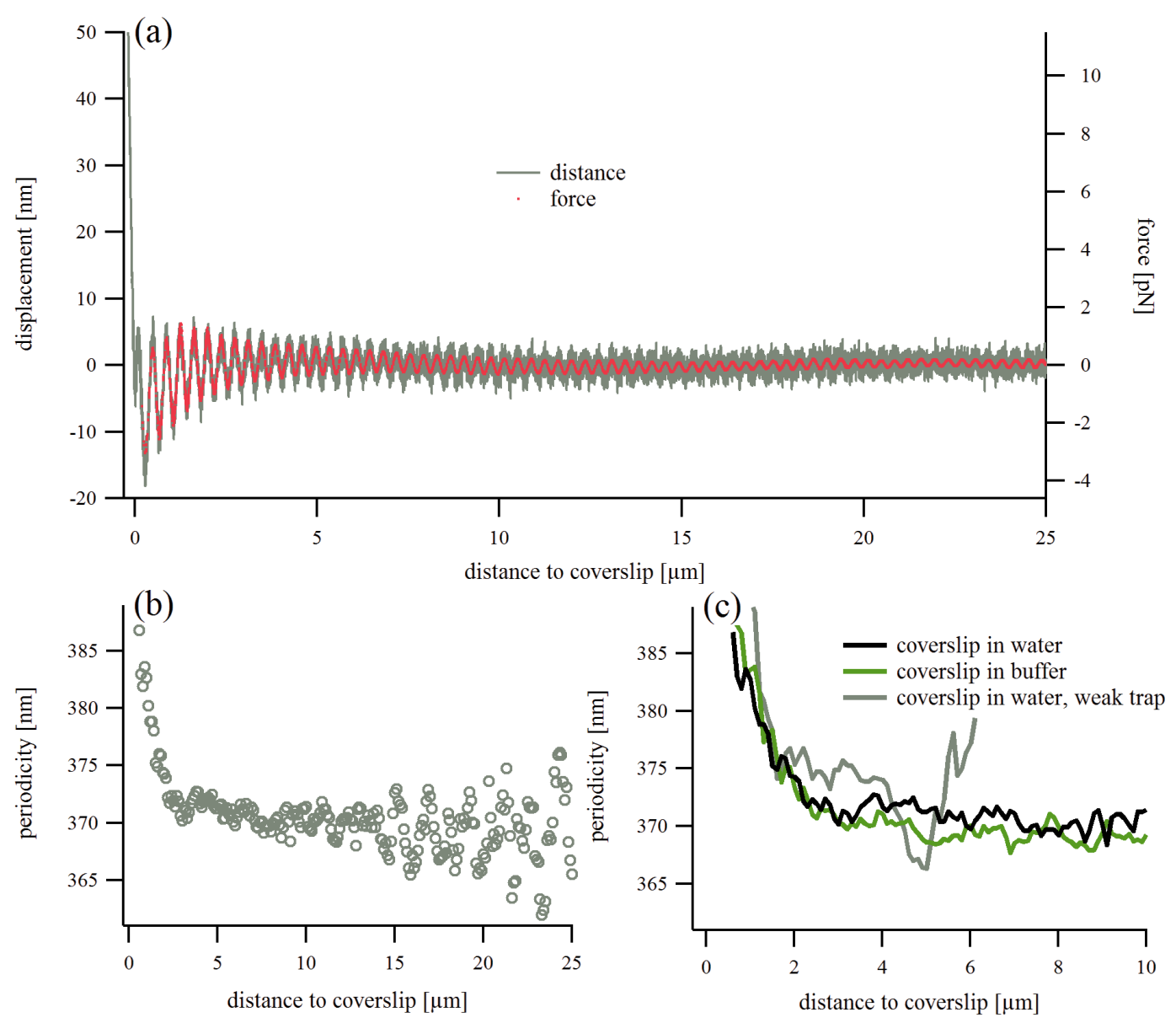

Figure 4.27: (a) A trace from a single trapped bead shows a periodic fluctuation with its amplitude increasing when the bead approaches the coverslip. The zero distance is defined as the position where the bead hits the surface and the displacement rapidly increases. The force (right axis) is obtained by multiplying the displacement with the spring constant of the trap. (b) The local periodicity was obtained by a fitting procedure (Eq. 3.4) for 12 different beads in water that were approached to the coverslip. The trap stiffness is $\approx 0.2 p N / n m$. Close to the surface the periodicity is $\approx 385 \mathrm{~nm}$. When the distance increases, the periodicity quickly decreases and converges to $\approx 370 \mathrm{~nm}$. Due to the decreasing amplitude of the interference signal, the fitting results become noisier when the distance increases. (c) The periodicity was also measured in buffer of high ionic strength (Krebs-Ringer solution) when the coverslip was approached (green, $n=4)$. The gray curve $(n=11)$ was performed with a three times lower trap stiffness but shows an identical trend. The noise is higher because the interference signal is less pronounced in the weaker trap.

When the vertical optical trap is used to perform deformation experiments on biological samples, this interference effect can lead to significant artefacts in the recorded signal. For experiments performed on cells, this problem, however, can be neglected since the cells own heights between 5 and $10 \mu \mathrm{m}$. At this distance 
the interference signal is reduced to single $\mathrm{nm}$. The interference effect can be further reduced by simply lowering the trap stiffness such that the bead has a higher mobility in the vertical direction. However, a lower trap stiffness also limits the maximum force that can be exerted. An alternative solution to reduce the interference is to reduce the reflectivity of either the coverslip or the bead. This can be achieved by antireflective coatings [82].

\subsubsection{Focus shift}

To confirm that with the use of a water immersion objective the shift in focus is indeed eliminated, we made use of the periodicity of the interference signal [83], [84]. To obtain the local periodicity of the interference signal, we splitted the traces as shown in Figure 4.27(a) in segments of $1110 \mathrm{~nm}$ (see equation 3.4), and fitted those with a sinusoidal function. Figure 4.27(b) shows that at a distance of $2 \mu \mathrm{m}$ or more away from the coverslip, the periodicity is $\approx 370 \mathrm{~nm}$. In the absence of a focus shift the expected periodicity of our $974 \mathrm{~nm}$ laser source in water is $\approx 367 \mathrm{~nm}$ $\left(n_{\text {water }}\right.$ at $\left.974 n m \approx 1.326[85]\right)$.

The constant periodicity confirmes a negligible focus shift away from the coverslip in our system. Interestingly, when the bead was moved close to the surface, the periodicity of the interference signal increases to $\approx 385 \mathrm{~nm}$ at $1 \mu \mathrm{m}$ distance. This finding indicates that either: (i) an actual focus shift exist when the trap is brought close to the surface, or (ii) that the bead is pushed out of the trap center by a repellent force originating from the surface. Recently, it had been shown that the hydrodynamic effects caused by the proximity of a surface effect the Brownian motion of the bead at distances of up to multiple micrometers from the surface [86]. This shows an interaction between the surface and the bead at these length scales still to be present. Moreover, Figure 4.26 displays that the hydrodynamic drag on the bead affects the calibration of the trap stiffness up to a distance of $20 \mu \mathrm{m}$ away from the surface. To record the interference effect, the bead was moved at very low speeds $(0.5-2 \mu \mathrm{m} / \mathrm{s})$ towards the surface. The drag forces on the bead resulting from this motion be in the femtonewton range only. They cannot explain the observed axial bead displacement of $15 \mathrm{~nm}$ at $1 \mu \mathrm{m}$ distance. To test for electrostatic mediated repulsion from the surface that could push the bead out of the trap center, the experiment was repeated in a buffer of high ionic strength 
$(0.14 M)$. Notably, no change of this effect could be observed (Figure 4.27(c)). To rule out any repulsive forces from the surface, the experiment was repeated using a three times lower spring constant. If repulsive forces played a role, using a weaker trap would increase the observed displacement. However, again the interference was not affected (Figure 4.27(c)). We conclude that the increase observed in periodicity must originate from a shift of the trapped bead away from the surface. This occurs when the trap is brought to the surface within $2 \mu m$ distance. Although detectable with $15 \mathrm{~nm}$ at $1 \mu \mathrm{m}$ distance, the shift is relatively small.

\subsubsection{Discussion}

The constructed vertical optical tweezer instrument can perform measurements at low force ranges $(1-50 p N)$. Compared to AFM it has the following advantages: It can work with a lower force set point since the inherent force noise of the force probe is smaller than in AFM. Thus, low force spectroscopy and probing of macromolecule dynamics is less disturbed than with AFM.

E.g. the measured RMS force noise of the tweezer probe at a detection bandwidth of $90 k \mathrm{~Hz}$ is much lower with $0.2 p N$ than the $20 p N$ for a low noise AFM cantilever. For example, the AFM cantilever noise would overcome the forces kinesin motor proteins can produce $(5-8 p N)$ [87]. Moreover, the finding that a ribosome is able to move on mRNA against a force of up to $20 \mathrm{pN}$ [88] can probably not be revealed within the AFM noise limits. Even the softest cantilevers have a spring constant of $\approx 0.03 \mathrm{~N} / \mathrm{m}$, which means that a small $1 \mathrm{~nm}$ error in the feedback loop that controls the position of the cantilever (and the indentation of the sample) will already propagate to a force error of $30 p N$.

Noteably, low force probing is not only of interest in the spatial regime of macromolecules. Small forces are also required to induce deformations smaller than those by the limits of AFM force noise. The deformation length scale determines the mechanical response of the cell: small deformations, such as performed with the vertical optical trap, result in a more elastic response and larger deformations performed with AFM in a more viscous response, [89]. When small probes (diameter around a $\mu \mathrm{m}$ ) are used to investigate the local mechanical response of the cell, a small force of $10 p N$ already leads to a $0.2 \mu \mathrm{m}$ indentation. Mechanical probing at this force / indentation regime can be well accessed with optical tweezers. With 
AFM only forces of at least $\sim 40 p N$ can be reliable used which results in a much higher indentation. An example for low force indentation is shown in fig. 4.28. We applied a force of $10 p N$ on fibroblasts, which results in indentations of 100 to $250 \mathrm{~nm}$.

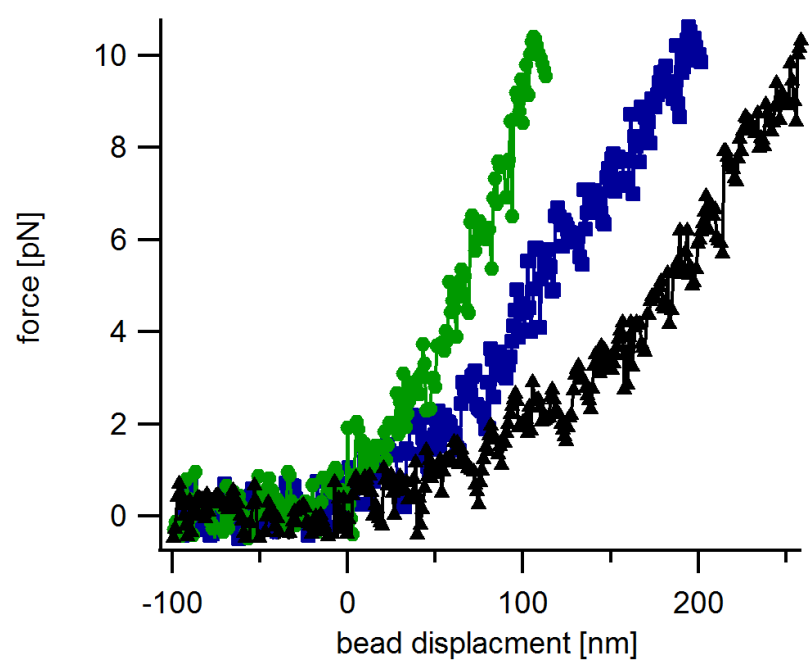

Figure 4.28: Low force indentation. Typical indentation curves on fibroblast cells. The cells were indented between 100 and $250 \mathrm{~nm}$ at a force of $10 \mathrm{pN}$. This indentation region can be well accessed with optical tweezers. For AFM this region is however hard to access. The large variation between the curves is inherent to measurements on cells.

To sum it up, an optical tweezer instrument could be shown to be a valuable instrument when force-noise of the AFM needs to be overcome. Therefore, it might be of valuable interest for future experiments of different research fields. 


\subsection{The application of the AFM-TIRFM setup on biological samples}

\subsubsection{Cell mechanics and Parkinson's Disease associated LRRK2 mutations}

\subsubsection{Introduction to experiments}

To explore the effects of LRRK2 on the mechanical structure of the cell, a LRRK2 variant was expressed in a $\mathrm{H} 4$ cell line $\mathrm{i}^{\mathrm{ii}}$. The expression of LRRK2 in the cells was visualized by labeling it with GFP. In fluorescence microscopy observations we have noticed a mixture of different expression patterns. Different cells present different cytoplasmic structures although they are from the same cell line (fig.4.29).
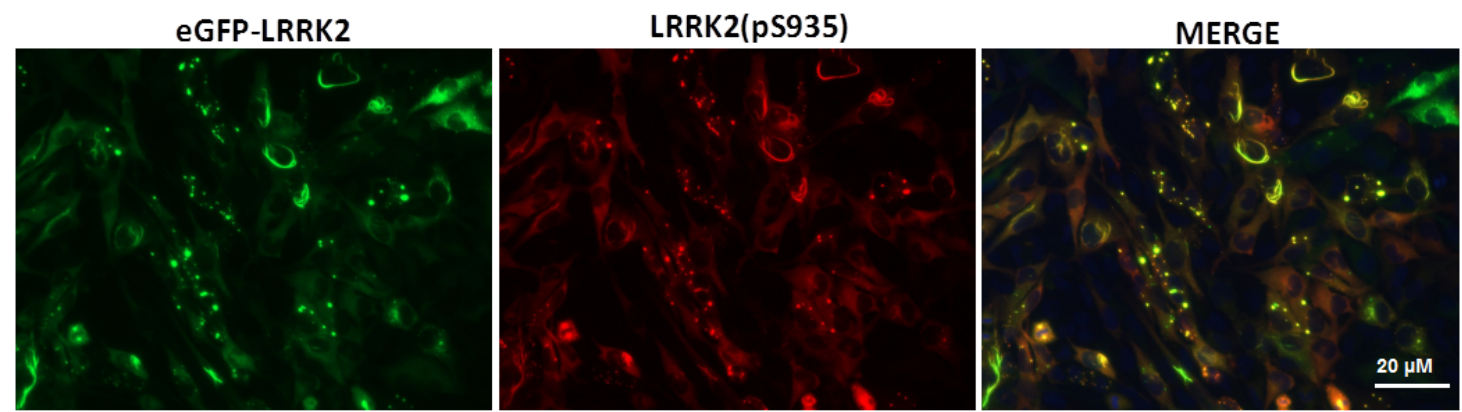

Figure 4.29: Expression of eGFP-LRRK2 in H4 cell line. H4 cells transduced with eGFP-LRRK2 lentivirus stably express LRRK2. The fluorescence microscope images show the different cytoplasmic expression pattern of LRRK2. Immunostaining revealed those to be highly phosphorylated. Images were taken by P. Guerreiro.

These findings lead to speculations about changes in cytoplasmic crosslinking, aggregation, depolymerisation, and polymerisation. In order to closely analyze the different intracellular patterns, we divided the cells into three fluorescence sub-types: uniform, aggregates, and filaments (fig.4.30). Earlier efforts to isolate these sub-types with cell biological methods had failed. To investigate a possible correlation between mechanical properties and fluorescence sub-type of the cells, the presented combination of AFM and TIRFM was applied. The TIRFM part

\footnotetext{
ii The cells were kindly provided by Patricia Guerreiro from the group of Prof. T. Outeiro at the Georg-August-Universität Göttingen.
} 
was used to identify the fluorescence sub-types and the AFM part to perform mechanical probing.
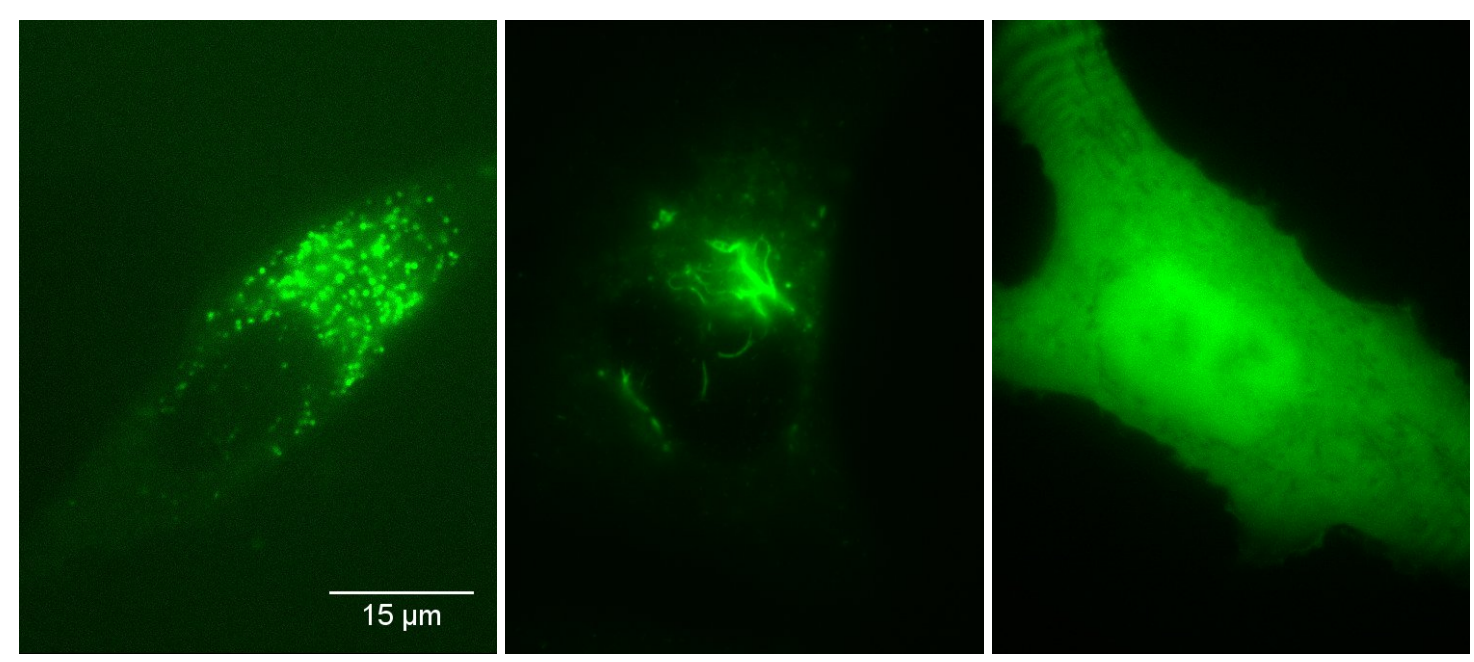

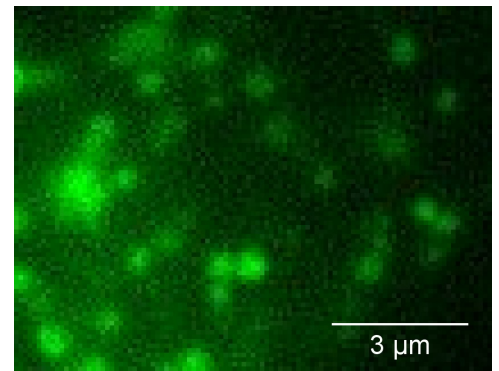

(a) aggregates

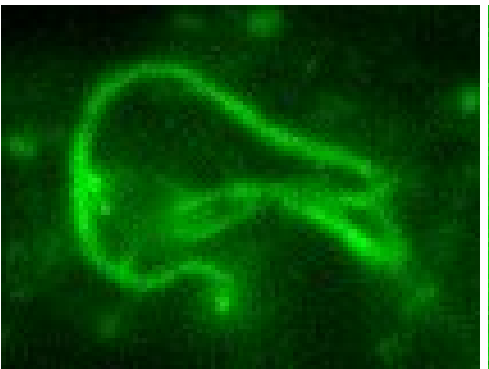

(b) filaments

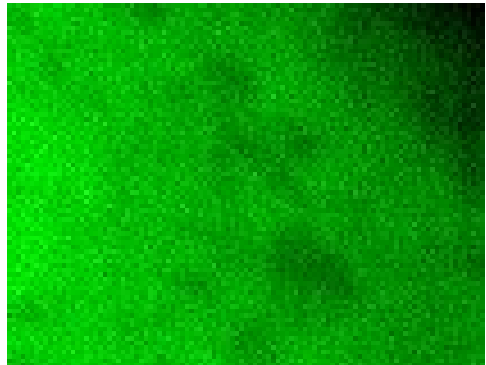

(c) uniform

Figure 4.30: The distinct fluorescence sub-types of the H4 cells expressing a GFP labeled LRRK2 variant.

The probing was performed via indention experiments. The cantilever, with an attached sphere was brought into contact with the cell until a certain force set point was reached. Then, the cantilever was withdrawn to the starting point. Effectively, the loading force vs. indentation relation was measured. In combination with the Hertz model (section 3.5) this delivers the Young's modulus $E$ as a characterization parameter. However, due to the cell structure it does not represent a purely elastic behavior but likely includes a viscous component as well.

\subsubsection{Indentation data analysis}

The AFM indentation experiments aim to evaluate mechanical differences between the three fluorescence sub-types (fig.4.30) and two control groups. The control 
groups include non-transfected $\mathrm{H} 4$ cells and cells that were transfected only with a GFP tag. The analysis shows that the Young's modulus of the uniform and filaments fluorescence sub-type is significantly higher than that of the sub-type aggregates and both control groups, fig. 4.31.

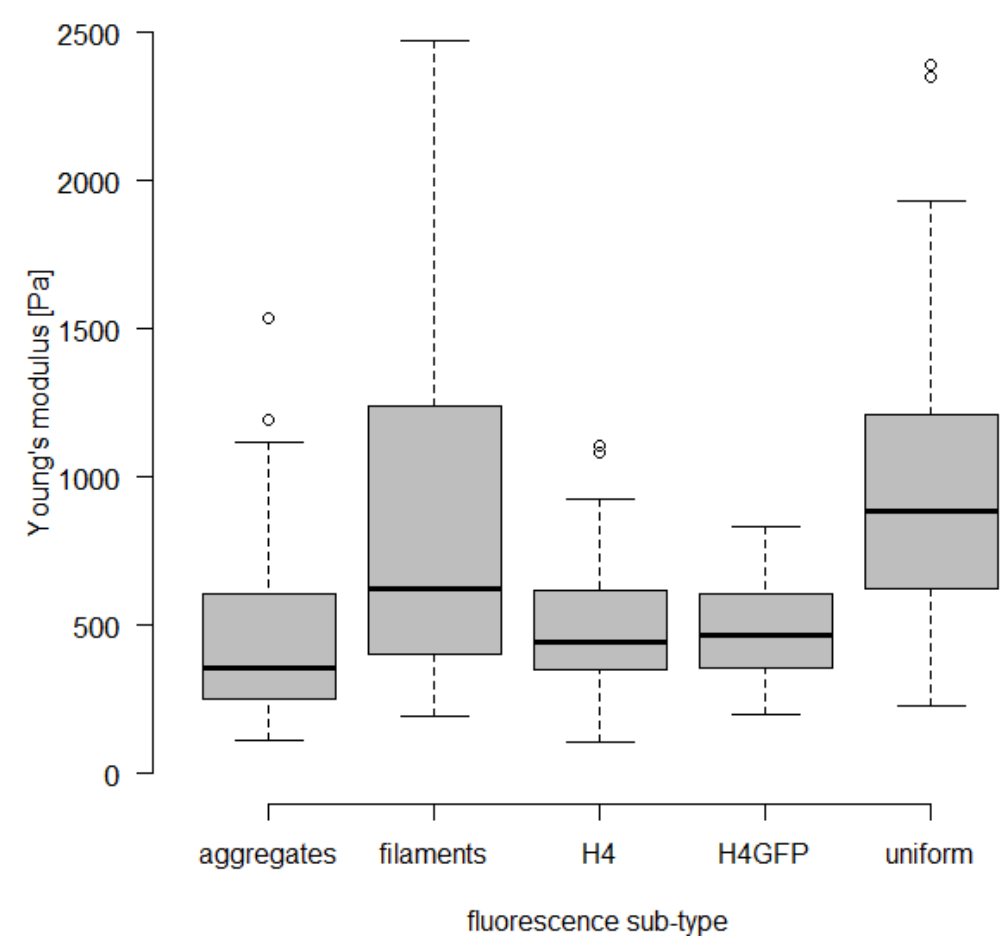

Figure 4.31: Boxplots, main effect fluorescence sub-type: the uniform $(\mathrm{N}=13)$ and filaments $(\mathrm{N}=16)$ type show significant differences in their Young's moduli compared to the type aggregates $(\mathrm{N}=18)$ and the two control groups $(\mathrm{N}=50)$.

The fluorescence sub-types were measured at three different indentation speeds (section 3.5.2.2). The analysis is based on a 2-factorial analysis of variance (ANOVA) with the inter-sample factor fluorescence sub-type and the intra-sample factor indentation speed. It was applied to test whether the observed differences of the Young's modulus can be attributed to the fluorescence sub-type, the used indentation speed, and/or an interaction of both factors. We found a significant main effect of the factor fluorescence sub-type, which is given with $F(2,44)=3.93, p<$ 0.05. This justified to perform post-hoc T-tests between the fluorescence sub-type 
groups. The total paring possibilities and their T-test results are shown in tab. 4.3.

Table 4.3: Post-hoc $\mathrm{t}$ tests between the three fluorescence sub-types. *: significant differences.

\begin{tabular}{l|lll} 
& uniform & filaments & aggregates \\
\hline $\mathbf{H} 4$ & $\mathrm{t}(13.16)=3.085$ & $\mathrm{t}(16.68)=2.17$ & $\mathrm{t}(23.36)=0.052$ \\
& $\mathrm{p}<0.05^{*}$ & $\mathrm{p}<0.05^{*}$ & $\mathrm{p}=0.95$ \\
& & & \\
aggregates & $\mathrm{t}(18.31)=2.78$ & $\mathrm{t}(23.67)=1.92$ & \\
& $\mathrm{p}<0.05^{*}$ & $\mathrm{p}<0.1$ & \\
filaments & $\mathrm{t}(26.1)=0.79$ & & \\
& $\mathrm{p}=0.43$ & & \\
\hline
\end{tabular}

Moreover, the analysis also revealed a significant effect of the factor indentation speed $(F(2,88)=76.80, p<0.001)$. This means that an increasing indentation speed leads to an increasing Young's modulus, probably caused by viscous elements in the cell structure. Interestingly, a significant interaction effect indentation speed $\mathrm{x}$ fluorescence sub-type $(F(4,88)=3.11, p<0.05)$ was found as well (fig.4.31). It reveals that the fluorescence sub-types reacts differently on the indentation speeds.

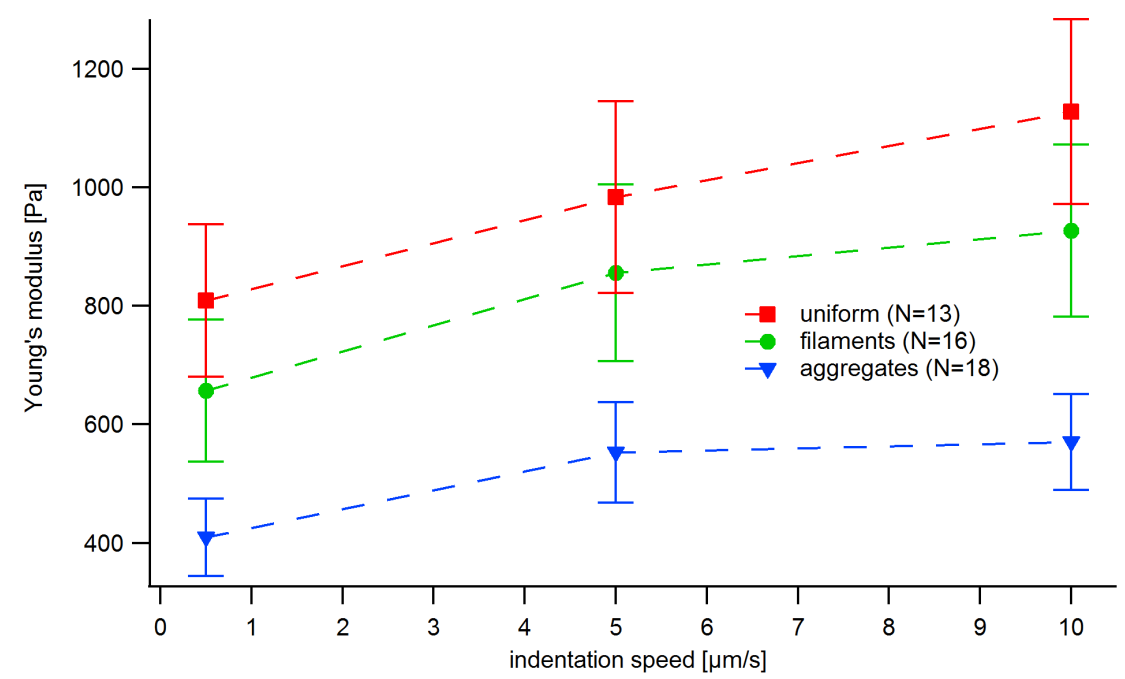

Figure 4.32: Both the fluorescence sub-types and the indentation indentation speed affected the measured Young's modulus. Error bars are SEMs. 
Whereas the uniform type shows an almost linear increase of the Young's modulus with the indentation speed, the Young's modulus of the types filaments and aggregates shows a much smaller increase above an indentation speed of $5 \mu \mathrm{m} / \mathrm{s}$. The data shown in fig. 4.32 visualize the effect of the indentation speed on the Young's modulus.

\subsubsection{Discussion}

The interaction of LRRK2 with the cytoskeleton in Parkinson's Disease (PD) is a widely adopted phenomenon. It originates from the discovery of LRRK2 interactors that represent cytoskeleton building blocks. The interactors including $\alpha / \beta$-tubulin could be linked to reduced neurite outgrow in different studies [90], [91]. Accumulations of hyperphosphorylated microtubule related protein Tau possibly cause effects on microtubuli dynamics [92], [93]. The actin network might be influenced by LRRK2 interactions with actin and actin-related proteins as well [32]. Furthermore, direct structural effects like microtubuli bundling induced by LRRK2 interactions with other proteins are reported [94]. Also regulations in anchoring the actin cytoskeleton to the plasma membrane were found to be induced by LRRK2 [95].

We investigated the relation between the three different expression patterns of LRRK2 and the cell mechanical properties. The TIRFM was used to identify the different fluorescence sub-types: uniform, filaments, and aggregates. Subsequently, the AFM was used to measure the Young's modulus of the cells by indentation experiments. The results reveal a correlation between the different fluorescence sub-types and their Young's modulus. The Young's modulus shows significant higher values for the sub-types uniform and filaments compared to the sub-type aggregates. The Young's modulus of the the sub-type aggregates shows no significant differences compared to the healthy control cell groups.

One of the pathological hallmarks of PD is the presence of inclusions composed by misfolded proteins (Lewy bodies) in the remaining neurons. Here, LRRK2, besides $\alpha$-synuclein, is often identified as one of the inclusion components [96]. The role of these inclusions, which may correspond to the fluorescence sub-types aggregates, has been extensively discussed. Whether they are toxic to the cells or protective is still unclear [97], [98]. 
The fluorescence sub-types uniform and filaments show higher stiffness values. Here, LRRK2 is spread through the entire cell and may compromise the cytoskeleton organization. The fluorescence sub-type filaments might reflect a LRRK2 induced bundling interaction via microtubule-related proteins. For the uniform sub-type an effect on the connection between actin cytoskeleton and plasma membrane would be imaginable. If the observed aggregations are indeed related to inclusions in forms of Lewy bodies, the following assumptions can be made on the basis of the described findings: The Young's modulus of the fluorescence subtype aggregates is not altered compared to the healthy control cells. Hence, the aggregated pattern might indicate an alternative way of the cells to compensate the excess of free cytoplasmic LRRK2. Thereby, interactions of LRRK2 with the cytoskeleton are prevented resulting in normal cell mechanics.

In the experiments on PD-like cells the AFM-TIRFM approach shows that different patterns of LRRK2 can be linked to different mechanical properties of the cells which is a first step in a mechano-functional analysis of LRRK2. Further experiments will be required to determine the molecular details of LRRK2 organization in the different subtypes.

\subsubsection{Actin binding protein DBN-1}

DBN-1 affects the actin filament organization and stability in the sarcomeres which are critical for force generation by muscle cells. In fluorescence microscopy it could be observed that in vitro DBN-1 bundles actin. The actin bundles tend to form loops with a significant higher probability than normal actin fibers. To investigate the structure of the loops, they can be traced using the TIRFM (fig. 4.33). Subsequent AFM imaging delivers structural details of the bundles. 


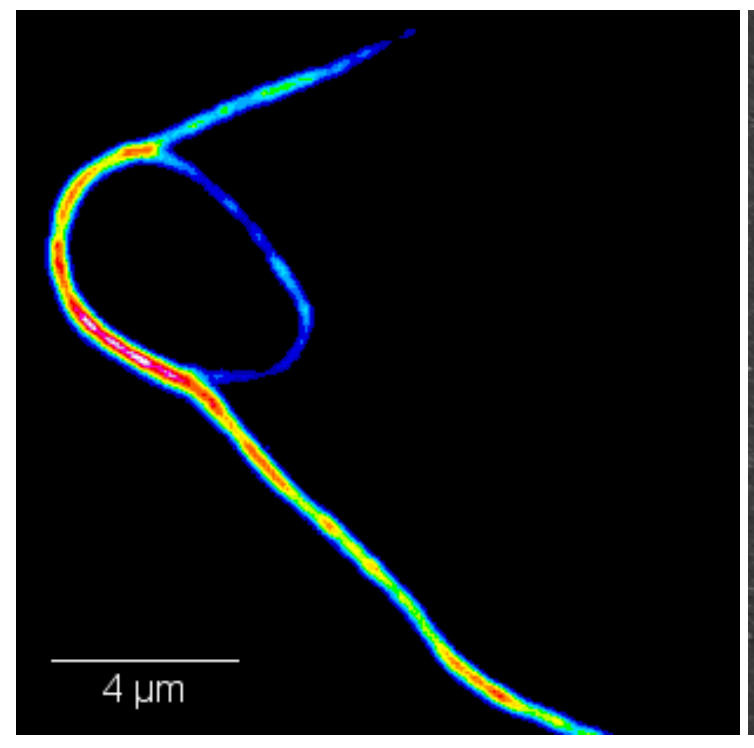

(a) Actin bundel, TIRFM

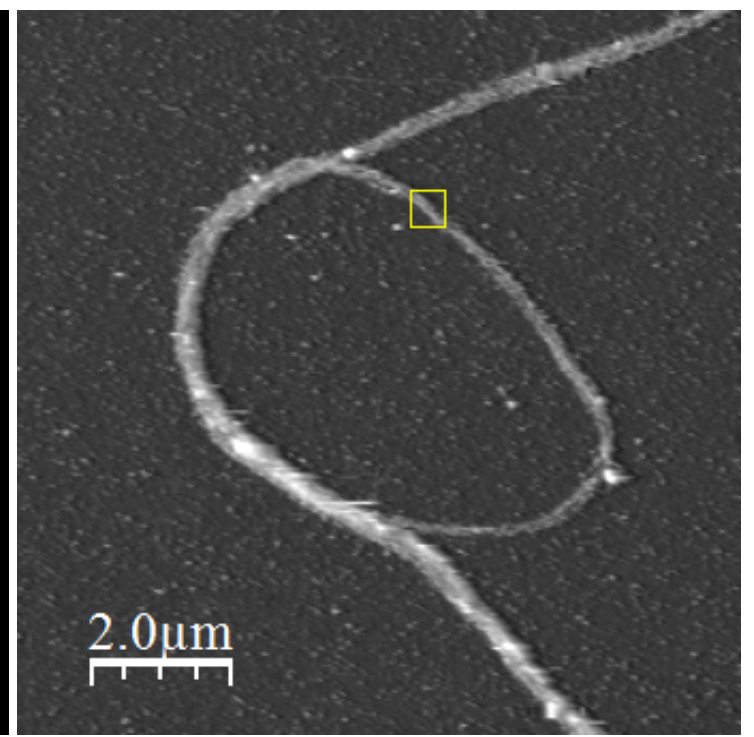

(b) Actin bundel, AFM

Figure 4.33: Direct visualization of actin filament loop formed in the presence of DBN-1. (a). Actin bundle loop formed in the presence of DBN-1, labeled with Rhodamine Phalloidin observed by fluorescence microscopy. (b). AFM image of the same actin/DBN-1 bundle loop. The yellow square marks the magnified region depicted in figure $4.34 \mathrm{a}$

The region in the yellow marked square of the actin bundle loop in figure 4.33b was imaged in more detail. The AFM imaging at single protein resolution shows that most of the actin/DBN-1 bundles in the loops contain 3-4, and in some cases up to 10, actin filaments. The DBN-1 molecules are localized along the of filaments, fig. 4.34a. 


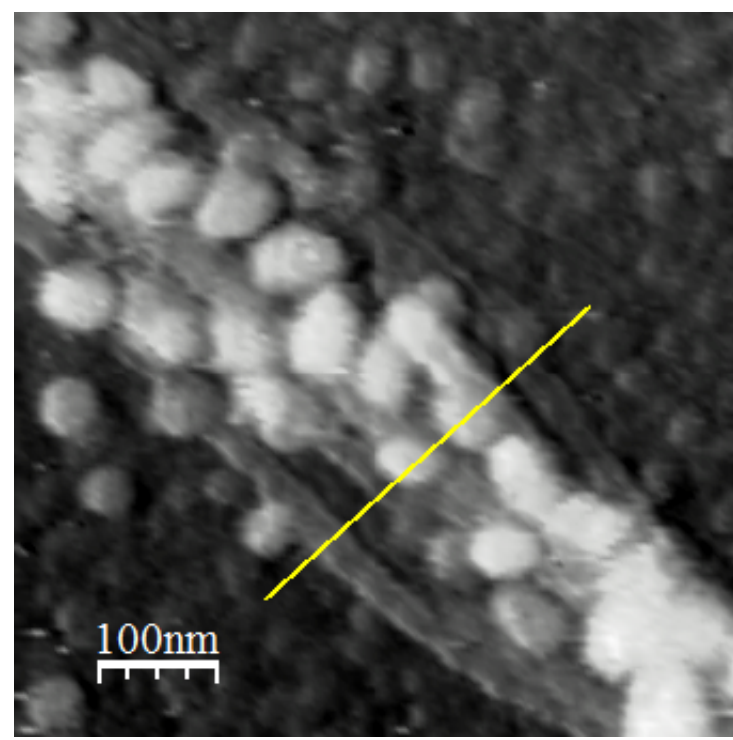

(a) actin fliaments plus DBN-1

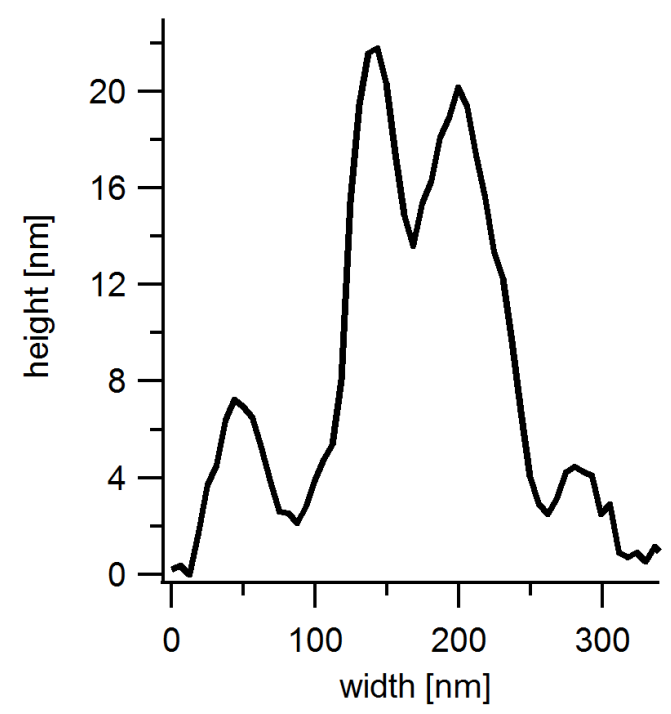

(c) bundle height

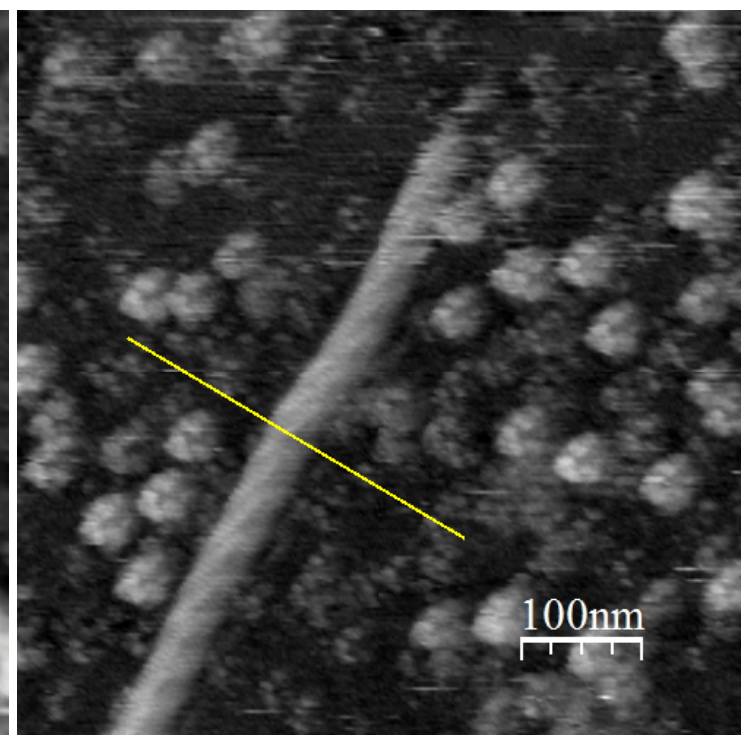

(b) actin filament only

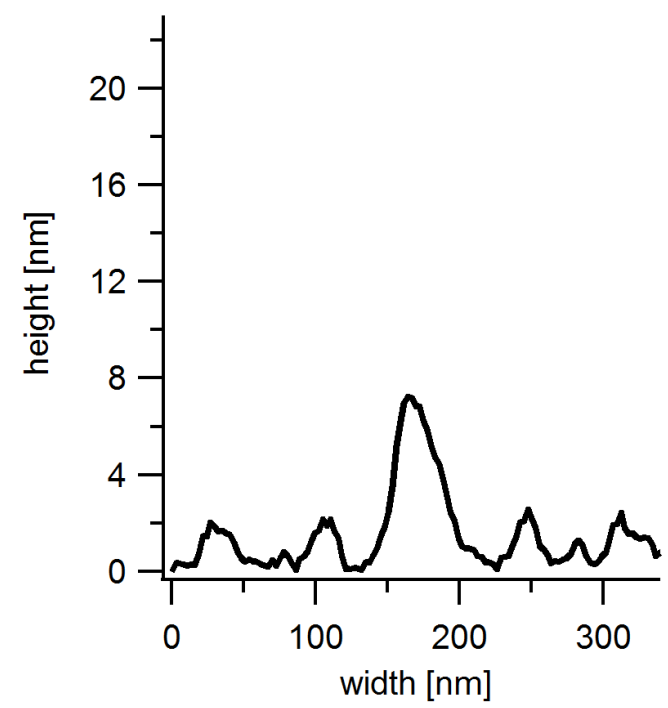

(d) single filament height

Figure 4.34: Detailed AFM imaging of actin fibers in presence and absence of DBN-1. (a): High magnification of the region depicted in fig. 4.33b. The loops contain bundles of several actin fibers. Single molecules of DBN-1 decorate the actin filaments. (b): AFM image of actin filament in the absence of DBN-1. The helical pseudo-repeat can be clearly distinguished. (c \& d): Height profiles along the lines in (a) and (b), with $\sim 20 \mathrm{~nm}$ the bundles are much higher than the individual $\sim 8 \mathrm{~mm}$ filaments.

The AFM imaging of several actin bundle loops allows to generalize the observations made in fig. 4.34: the bundles contain several filaments and DBN-1 is localized along the filaments, respectively the bundles. Herewith, we visually confirm the actin binding/bundling in the presence of DBN-1. The bundling likely 
occurs by DBN-1 cross-linking individual actin filaments. Interestingly, the diameter of the loops (around $\sim 17 \mu \mathrm{m}$ ) is significant smaller than the persistence length of F-Actin $(\sim 17 \mu m)$ [99], which suggests a stabilization of the loops by DBN-1 cross-linking. From our high resolution images we found, however, no evidence that binding of DBN-1 induces additional curvature in the actin filaments.

\subsubsection{Mechanical unpacking of Adenovirus capsids}

\subsubsection{Introduction to experiments}

To study the genome release of the Adenovirus, we induced a capsid dissasembly by an AFM force distance curve (single push). The genome spread is followed by fluorescence labeling and TIRFM. The labeling was done by using a DNA specific dye (YoYo-1) which increases its fluorescence $\sim 3000$ times when bound to the double-stranded DNA. The genome release can be observed in real time since the dye gains access to the DNA after capsid breaking. The experimental idea is sketched in fig. 4.35. The aim of this experimental configuration was to compare the genome release of mature WT and immature TS1 virons ${ }^{\text {iii }}$. Results of this in vitro experiments might contribute to a better understanding of the relevance of the maturation step for the efficient genome release during the infection pathway.

\footnotetext{
iii WT \& TS1 viruses were kindly provided by Dr. Maarit Suomalainen from the group of Prof.
} Urs Greber at the University of Zurich. 


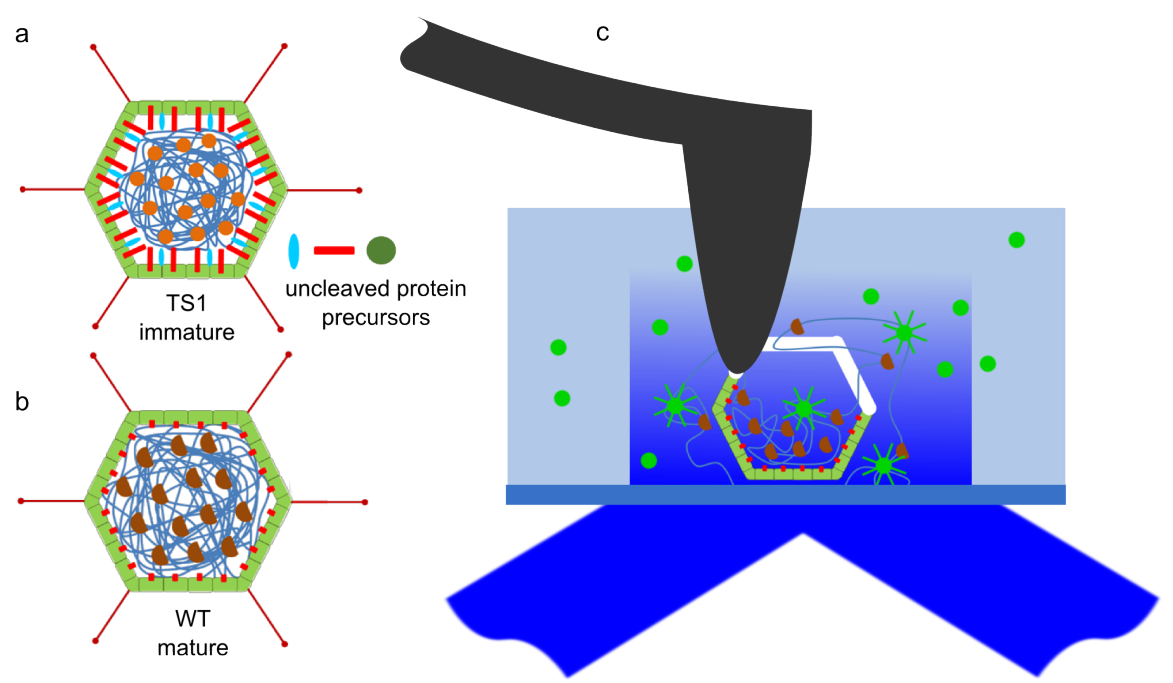

Figure 4.35: Mechanical unpacking of viral capsids. The basic idea is to induce capsid disassembly by a single force distance curve and to observe the genome release by fluorescence detection. Contrarily to continuous AFM image acquisition, the method does not induce additional spread of the genome by touching it repeatedly. The aim is to reveal genome release differences between a mature WT adenovirus and an immature TS1 mutant. The mutant contains uncleaved proteins which are speculated to keep the core in a more condensed state after capsid dissasembly.

\subsubsection{Mechanical fatigue and dye permeability of the adenovirus capsid}

First, we performed a mechanical fatigue experiment and explored the possibility of using fluorescence detection as a marker for capsid rupture. The virus capsid was repeatedly imaged in tapping mode (see section 3.6.4 for the conditions). During each scan the possibility exist that the sample gets damaged [48]. In our results we find that initial damage is visible after 5-10 frames and disruption is completed after $\sim 20$ frames. A TIRFM image after every AFM image was used to check for an eventual increase in fluorescence. Fig. 4.36b shows that the first penton is removed from the capsid at frame 7 . After this, a small increase in fluorescence can be detected (fig. 6.1a). Fig. 4.36c shows the clear breaking of the capsid at frame 17, which is followed by a massive increase in fluorescence fig. 6.1a. The results confirm that the DNA specific dye present in the buffer can only access the viral genome in relevant quantities after the capsid has been disrupted. Furthermore, the fluorescence detection turned out to be sensitive enough to log even a single missing penton from the capsid. 


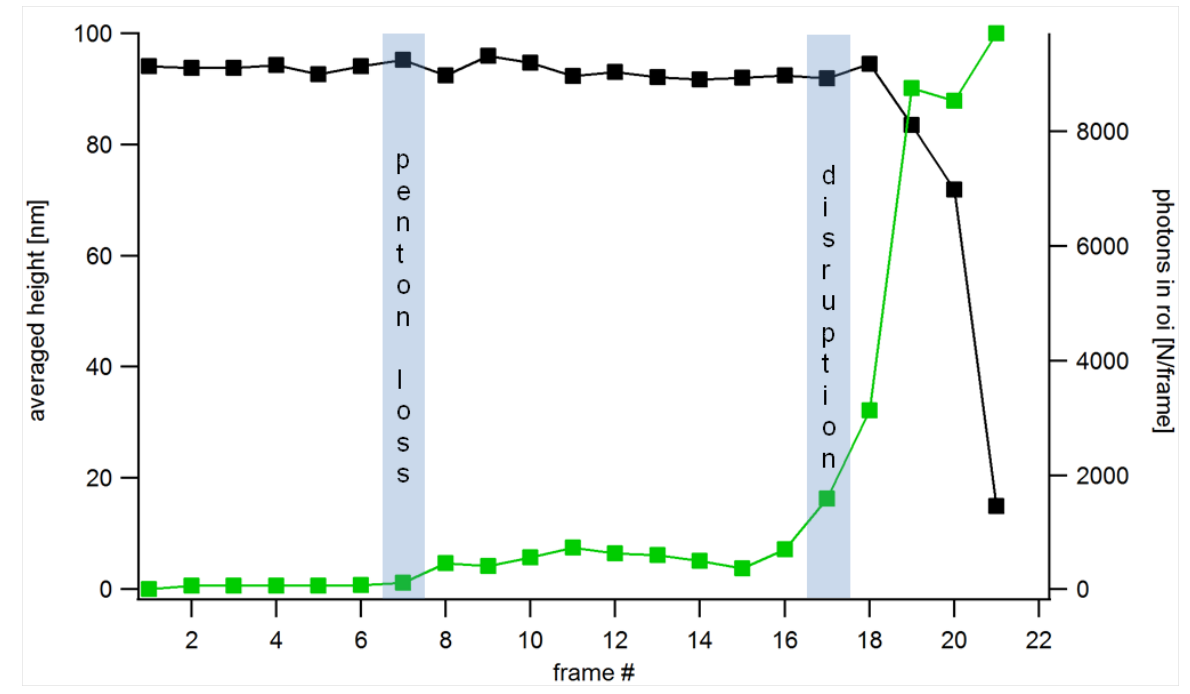

(a) height and fluorescence evolution

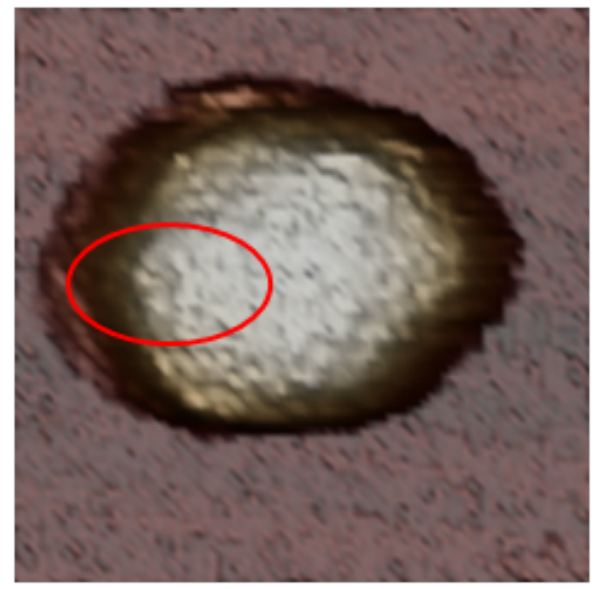

(b) penton loss, (frame 7)

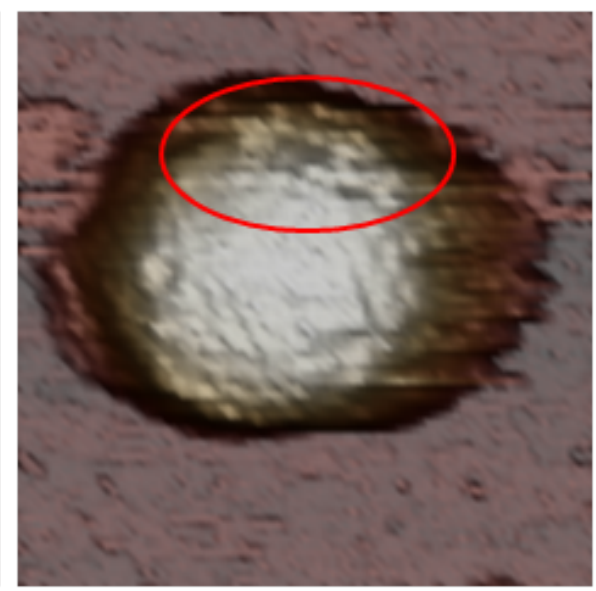

(c) shell disruption, (frame 17)

Figure 4.36: Mechanical fatigue experiment. (a): height and fluorescence of a viral particle vs. the number of acquired AFM images. The height was measured from every AFM image. After every AFM image, a TIRFM image was acquired to check viral shell integrity. On frame 7 a region indicating a missing penton can be seen. Interestingly, after frame 7 an increase in the fluorescence signal occurs, so the viral shell have must become permeable for the DNA specific dye present in the buffer. On frame 17 a region occurs which looks like a disintegration of the viral shell. The fluorescence signal after frame 17 rises strongly since the dye suddenly obtained access to a large amount of DNA binding places. (b): AFM frame 7. (c): AFM frame 17. All AFM images were acquired in tapping mode with the lowest possible set point. Dye concentration: $300 n M$. 


\subsubsection{Breaking force and spring constant}

In the next experiment we ruptured the viron particles with a single force distance curve performed at high force. The force distance experiments deliver two mechanical parameters of the capsid: the breaking force and the spring constant. The breaking force is indicated by the point where the continuous force increase breaks down. This sharp kink marks the shell rupture, fig. $4.39 \& 4.44$. The viral spring constant is obtained by fitting a linear function to the force vs. indentation relation between a force of $50 \mathrm{pN}$ up to the mentioned breaking point. Former mechanical investigations of the mature and immature particles reveal an increased capsid stiffness for mature virons $(\sim 121 \%)$ [43]. Since the viral core is decompacted by the processing of viral protease during maturation, higher stiffness might be related to an increase of the internal pressure [100]. The analysis of our collected data delivers a consistent result. The mature virons show a higher stiffness $(0.95 \pm 0.06 \mathrm{~N} / \mathrm{m})$ than the immature ones $(0.75 \pm 0.06 \mathrm{~N} / \mathrm{m})$, (fig. 4.37). Moreover, the breaking force is higher for the WT capsids $(8.23 \pm 0.5 n N)$ compared to the immature capsids $(6.31 \pm 0.4 n N)$. When the DNA specific dye is present in the buffer, the spring constant seems to be slightly affected. However, no common effect can be addressed, since the spring constant seems to be slightly increased for TS1 and slightly decreased for the WT group. The breaking forces remain largely unaffected by the presence of YoYo-1. 


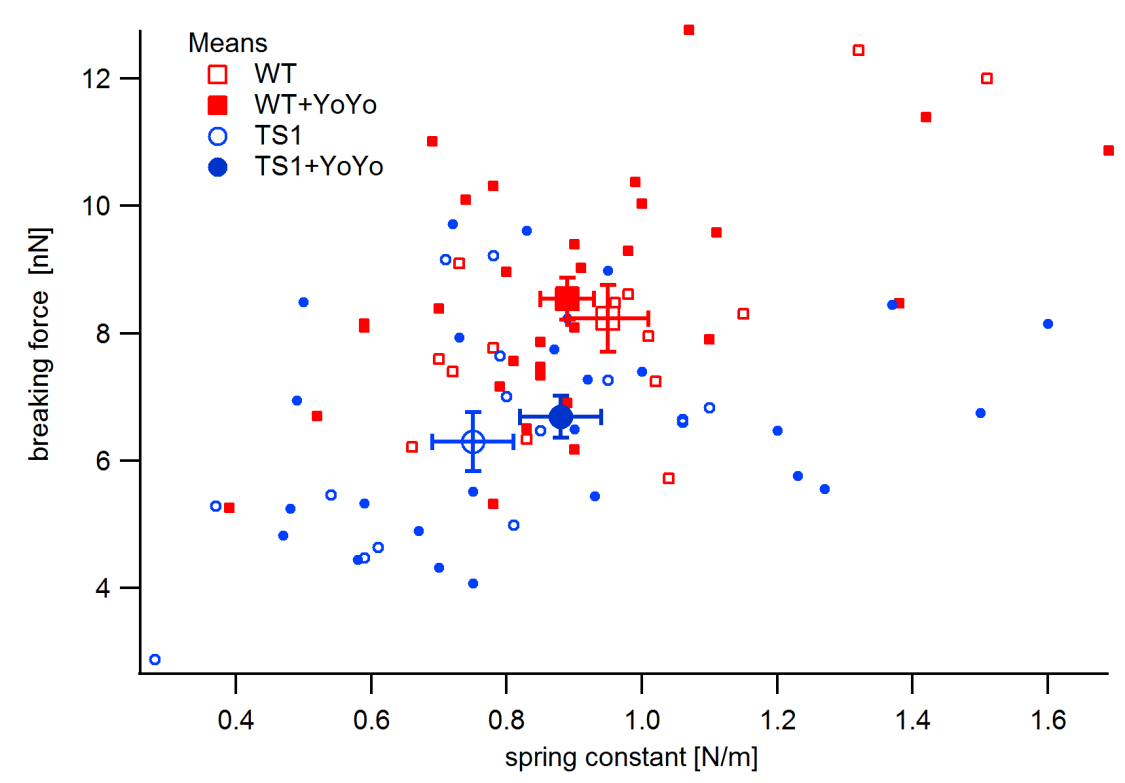

Figure 4.37: Breaking force vs. spring constant. WT \& TS1: the mature WT virons require higher breaking forces compared to the immature TS1 virons. Furthermore, mature particles have higher spring constants than immature ones. This is consistent with previous literature findings. The dye seems to have little effect apart from slightly stiffening TS1. Error bars show the SEM.

\subsubsection{Real time fluorescence detection during virus rupture}

The experimental course for single particle investigations started with an AFM overview scan of $5 \mu \mathrm{m} \times 5 \mu \mathrm{m}$ to localize a virus particle. The first viron was always ruptured before the dye was added to the buffer. This procedure served as control for sample preparation: intact virons, proper immobilization, and cantilever mounting. Afterwards the dye was added up to a concentration of $300 n M$ and the cantilever tip was co-aligned with the center of the field of view. Then a particle was selected, imaged at high resolution (fig. 4.38a), and a TIRFM image was taken (fig. 4.38c). After performing a force curve at high force $(10 n N)$, the fluorescence intensity was observed for $80 \mathrm{~s}$ (both data sets are shown further below). A fluorescence image of the destroyed particle is shown in fig. 4.38d. Finally, an AFM image was recorded, to confirm the disruption of the virus, fig. 4.38b. For imaging details please refer to the method section 3.6. 


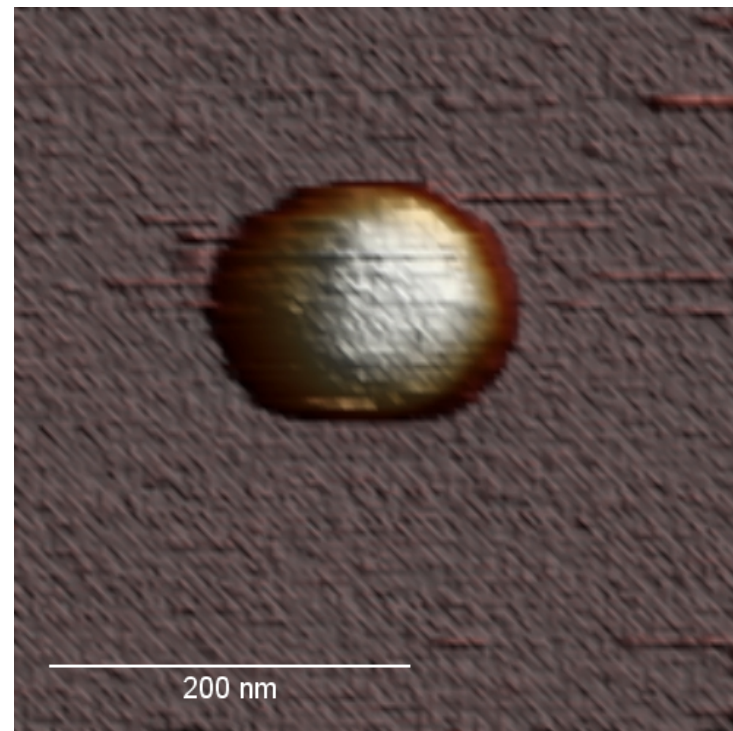

(a) intact viron

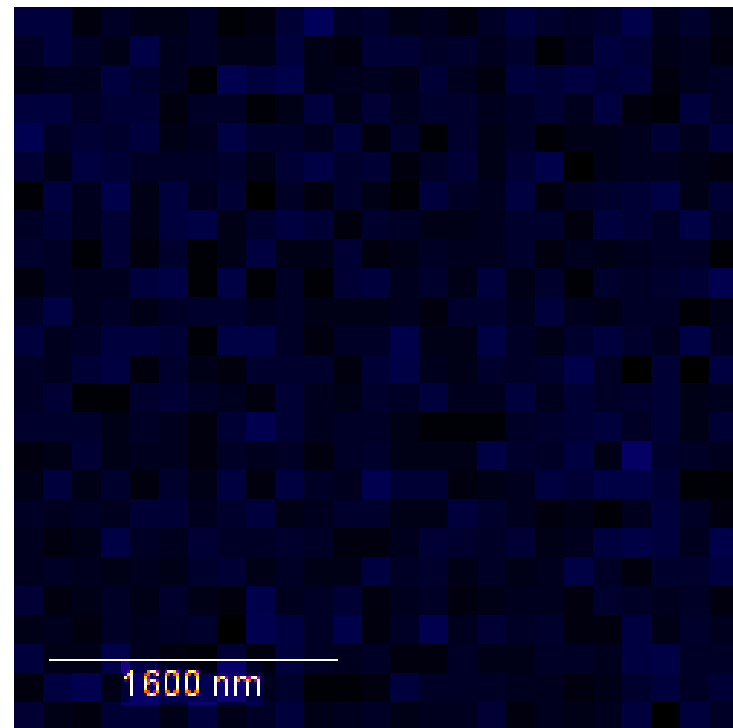

(c) intact viron

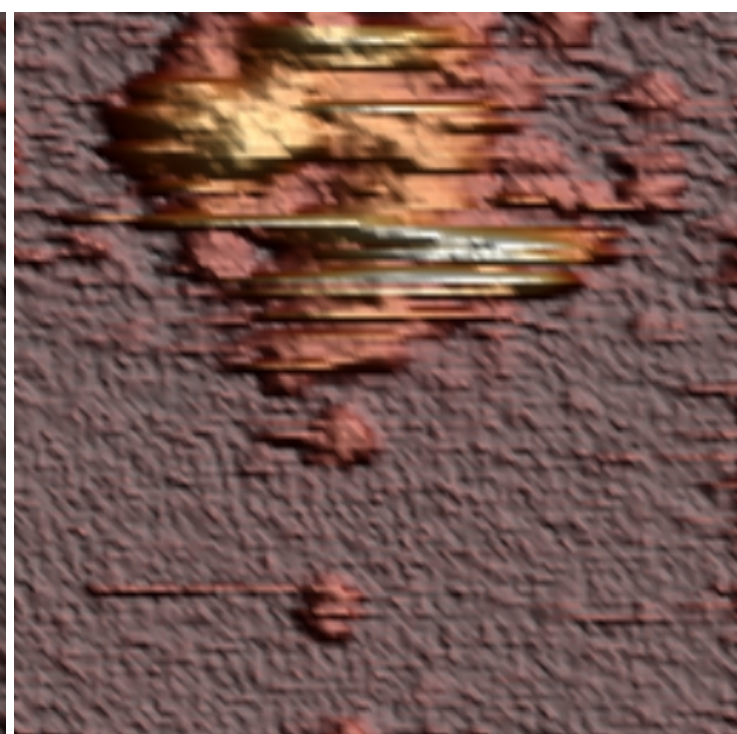

(b) after shell rupture

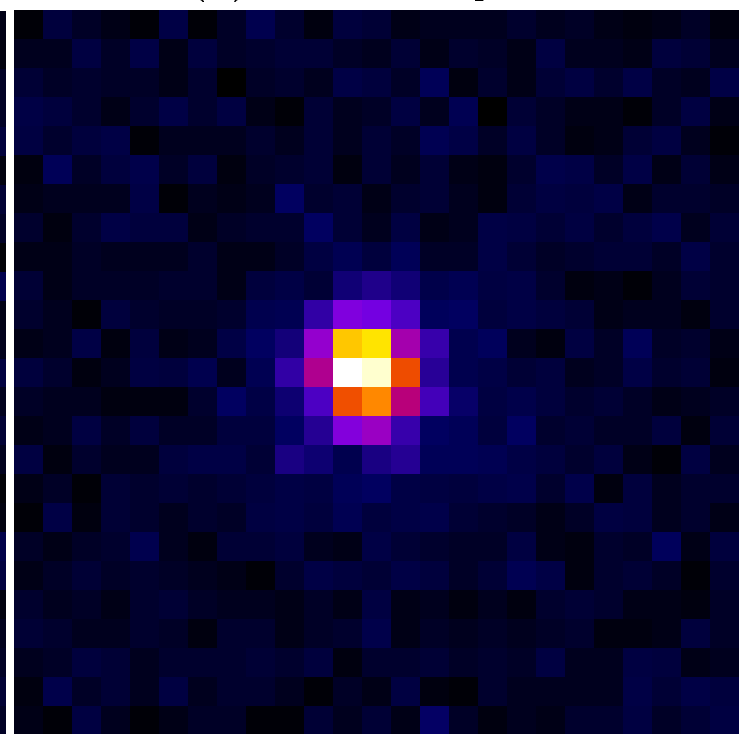

(d) after shell rupture

Figure 4.38: AFM (a \& b) and TIRFM (c \& d) images before and after induced virus shell disruption. In (a) the viron is adsorbed in a 3 -fold symmetry orientation. Only these orientations are stable enough for AFM imaging since the virus surface contact area is maximized. The viron is ruptured with a force distance curve at a force of $\approx 10 n N$. (c) same region as in (a), no fluorescence above the background level is detectable. (d) after capsid rupture the DNA specific dye binds and the genome becomes fluorescent.

The force vs. distance data acquired during capsid rupturing and the simultaneous detected fluorescence intensity allow to gain further insight into the mechanical unpacking process. The time course of the mechanical and fluorescence data are both plotted in figure 4.39. Here, it can be cleary seen that the virus shell rupture 
correlates with the fluorescence increase.

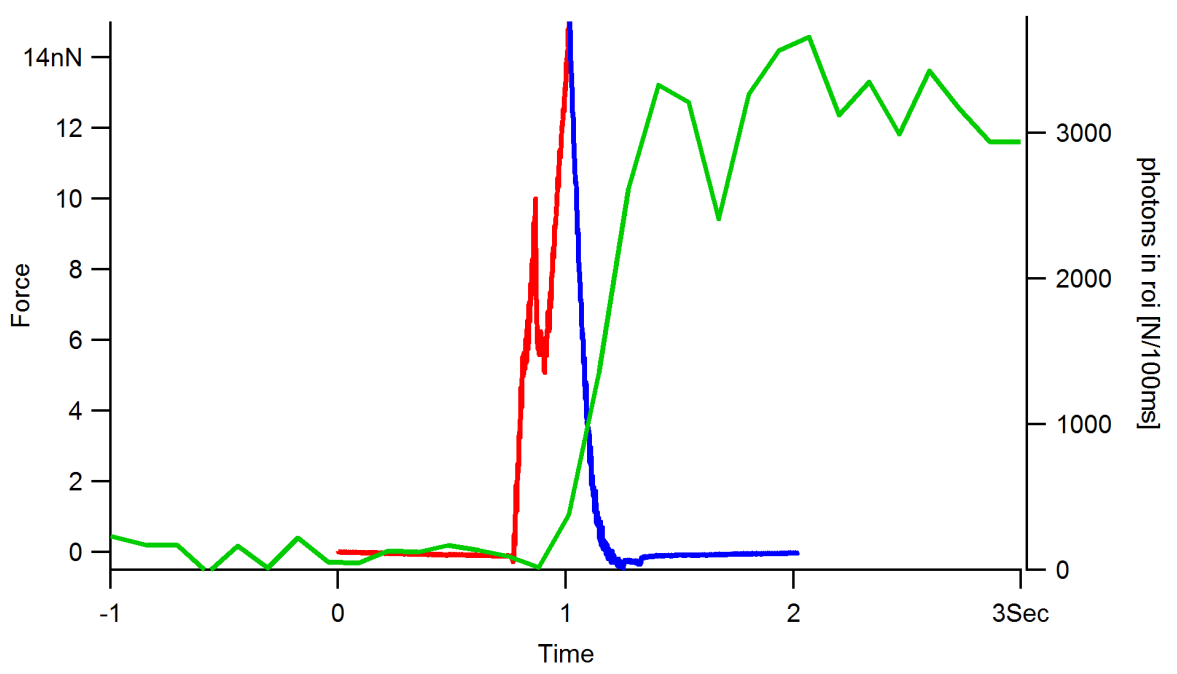

Figure 4.39: Virus capsid rupture and fluorescence increase. (Red): approach of the AFM tip towards the virus capsid. The force signal increases as the tip gets into contact. At $\approx 10 n N$ it shows a sharp kink which corresponds to the capsid rupture. After the force set point has been reached, the tip is withdrawn (blue). As soon as the shell ruptures, the dye can bind to the DNA and becomes fluorescent (green). The camera starts to acquire images $1 s$ before the force curve is started.

The introduced hypothesis about the TS1 genome remaining in a more condensed state than the WT genome was investigated as follows: The first parameter of interest is the evolution of the fluorescence intensity over time. This was recorded over a time of $80 \mathrm{~s}$ with an exposure time for a single frame of $100 \mathrm{~ms}$. As a general finding, intensity increases for the WT and slightly less for the TS1 particles over time. The inset in figure 4.40 shows the detected photons emitted from a single WT particle. To compare the WT group with the TS1 group, the number of detected photons for every particle is collected as a cumulative time trace. For every group the averaged time trace is plotted as presented in fig. 4.40. To connect this signal to a more or less condensed genome, one can think of the number of emitted photons as a value proportional to the number of bound dye molecules. The number of bound dye molecules depends on the number of available binding places. A more condensed genome will have less binding places avaliable for dye molecules. Therefore, the total number of emitted photons should be less. 


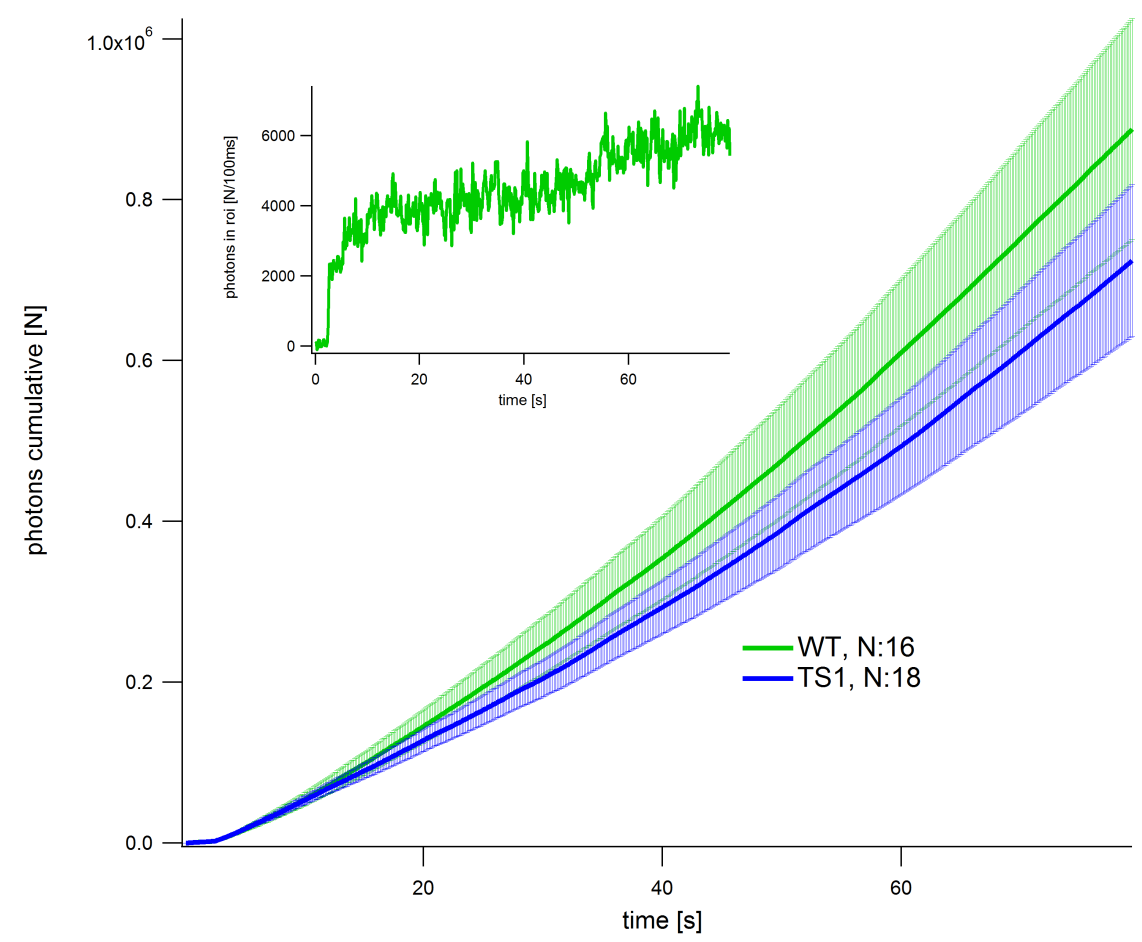

Figure 4.40: Emitted photons after capsid rupture for the WT virus and TS1 mutant. The inset shows the detected photons emitted from a single virus. The main plot compares the averages of the detected photons for the WT and the TS1 group over time. The photons are counted cumulatively since the total number corresponds to the bound dye molecules. This depiction includes dye molecules already bleached. Those are not taken into account in a photons per frame depiction (inset). Error bars are SEMs.

The comparison of the cumulative emitted photons does show indeed that more dye molecules bind to WT particles than to TS1 particles on average. However, the difference is small. The influence of the sample age (time from adding the dye to pushing an individual particle) could effect the number of available dye molecules. Therefore, we also looked at this influence and found a decrease of the emitted photons with increased sample age (data shown in the appendix section, fig. 6.1a). However, the sample ages for both groups are spread in the same way, so that this influence should level out.

As a second parameter we determined the spatial genome spread directly. A 2D-Gauss distribution can be fitted to the intensity distribution of the imaged particles. The width parameter (standard deviation) of this fit indicates the spatial spread. The details of the method are explained under section 3.6.1. Figure 4.41 illustrates radial averages of the intensity distributions of a WT and a TS1 particle. 
Both are fitted with a 1D-Gauss function.

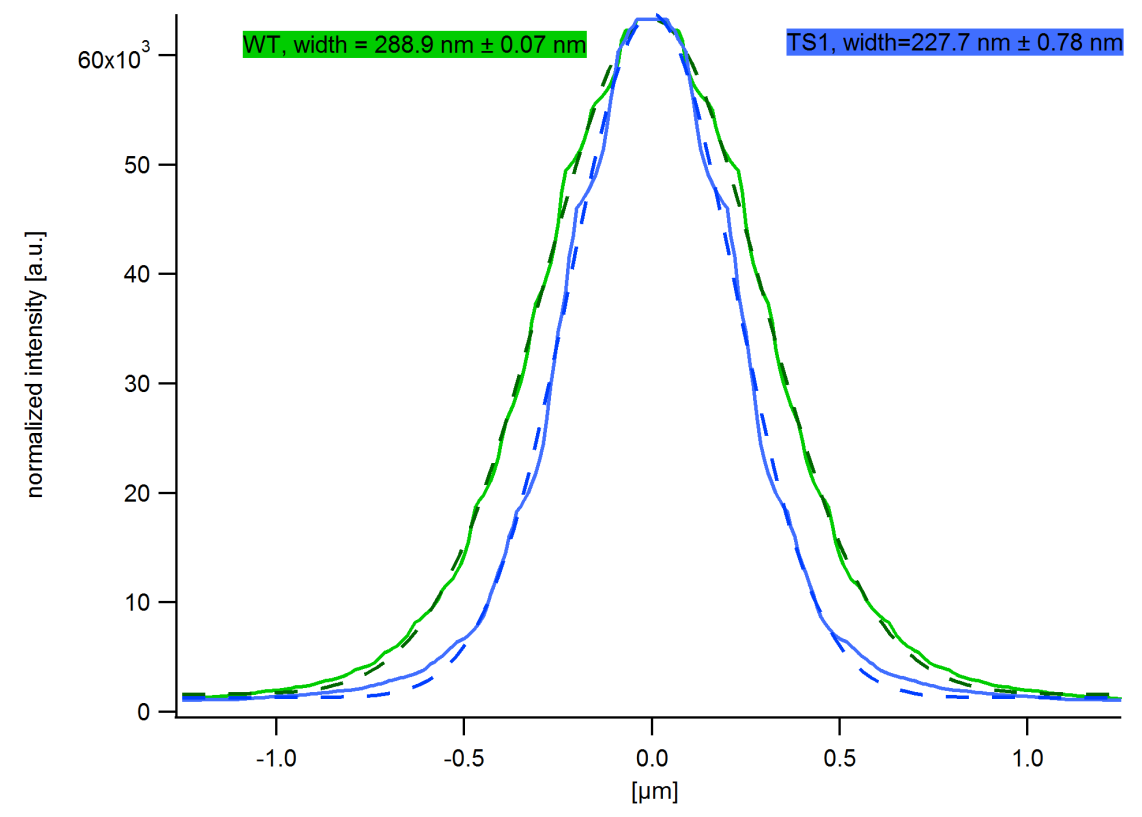

Figure 4.41: Radial averaged intensity distribution of a single WT and a TS1 particle (solid lines) fitted with a Gauss function (dashed lines). The width parameter of the fits reveal the spatial spread of the fluorescent dye molecules bound to the viral genomes (see also section 3.6.1). The chosen particles and their widths roughly represent the average values for the widths of the investigated WT and the TS1 group as presented in fig. 4.42 .

The indicated widths correspond to the mean values determined for the WT and the TS1 group. Figure 4.42 shows the average width values for both groups. A T-test supports a significant difference $(t(26)=2.9, p<0.05)$ between the width of the WT group and the TS1 group. The width value of a single particle is derived by fitting a 2D-Gauss to every frame of the 80 s movie that shows a fluorescent spot. Hence, in total, the individual particle average width is calculated from $\approx 580$ frames, respectively fits. The whole analysis was performed by a custom written program, see section appendix 6.2 . 


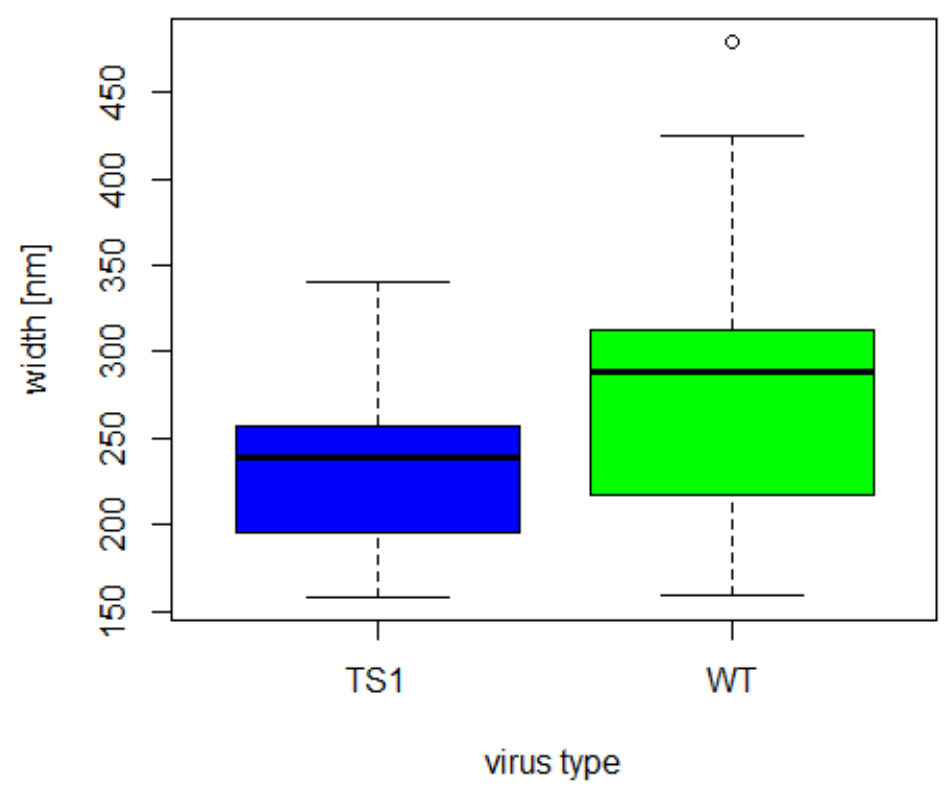

Figure 4.42: Boxplots, average width of the intensity distributions from WT and TS1 cores after viral shell rupture. WT $(\mathrm{N}=16), \mathrm{TS} 1(\mathrm{~N}=18)$. The width of the WT cores is significant higher than for the TS1 cores. This indicates that the WT genome stays less compact or spreads more after the shell has been disrupted.

Also for the width parameter, the correlation with the total emitted photons was investigated. No correlation could be found between both parameters. Hence, the differences must originate from the spreading behavior of the genomes. The scatter plot is shown in the appendix section, fig. 6.1b.

To investigate if the genome spreading is a dynamic event after capsid rupture we plotted the fitted width for each frame as a function of frame number (time), shown in figure 4.43. Here, every data point represents the average width of the WT group and the TS1 group at a certain time coordinate. Interestingly, the variance of the widths of the WT particles is larger than the variance of the TS1 group values during the first twenty seconds. This observation might indicate the viral content conglomeration to be looser/more diffusive. Variance decreases during the second half of the examined time period. This might be explained by the settling of loose genome parts on the surface or by the loss of loose DNA strands. Akerman \& Tuite reported photocleaving of single- and double-strand DNA strands by the YoYo class dyes [101]. The width of the TS1 particles seems 
to slowly increase over time. A possible explanation can be a slow ongoing core decompaction of parts which are not adsorbed on the substrate.

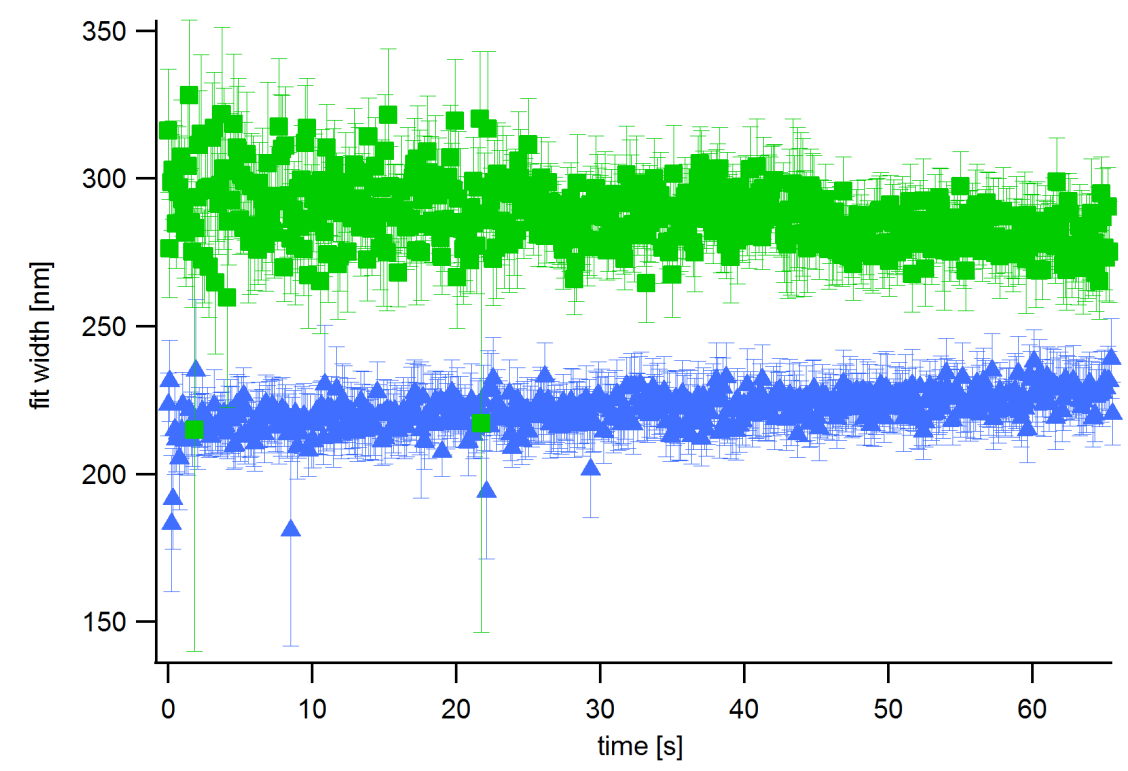

Figure 4.43: Average width of WT and TS1 particles vs. time. The average WT width decreases over time. This might be due to the diffusive loss of DNA or photocleavage of DNA strands by the bounded dye molecules [101].

The third parameter analyzed in the mechanical virus unpacking experiment was the fluorescence rising time after capsid rupture. Here, the idea is that the fluorescence increase of the TS1 genome could be slower since the dye molecules can access binding places only at the external periphery of the remaining core structure. In contrast, the less compact genome of the WT virus should offer more binding places such that the fluorescence signal should rise faster. Measuring the rising time requires the definition of distinct points over time. The first event is the moment of capsid rupture (sharp kink in the force distance signal). The second time point is defined by a certain fluorescence intensity level. This level is set by the mean fluorescence intensity before the tip contacts the viron (rising of force) plus twice its standard deviation (fig. 4.44). 


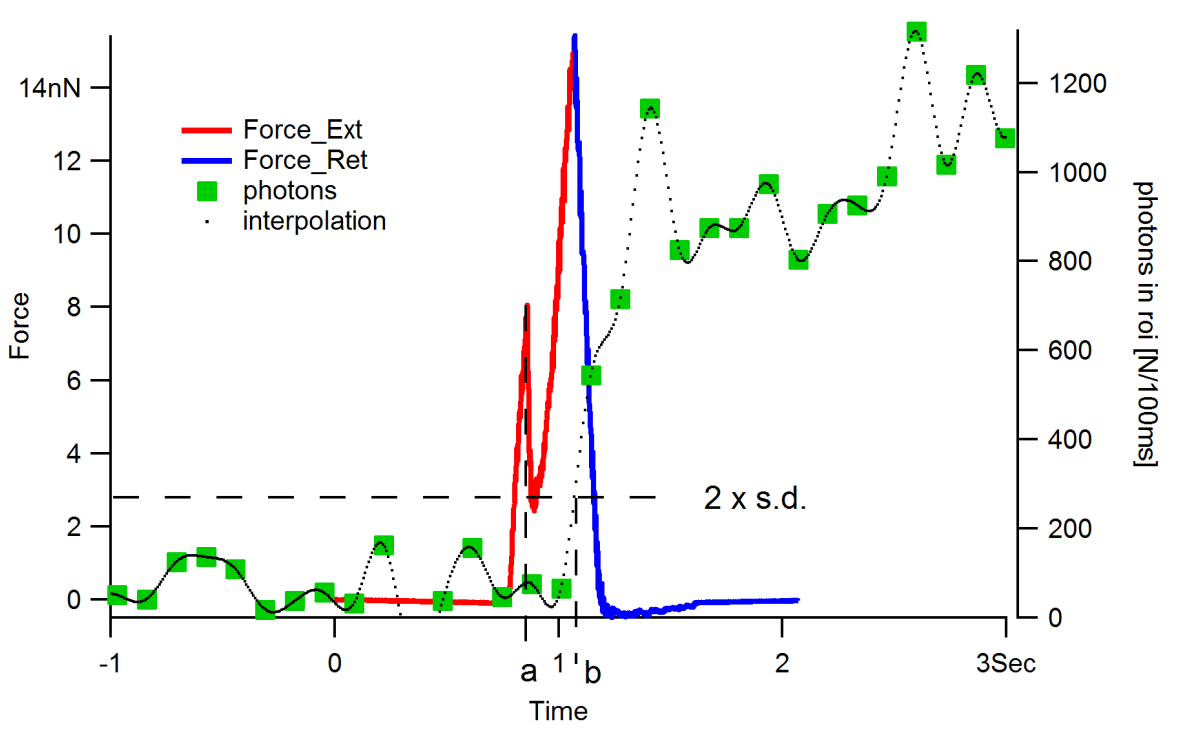

Figure 4.44: Measuring the fluorescence rising time after capsid rupture. The fluorescence rising time is defined as the time between the capsid breaking (kink in force distance signal (a)) and the time where the fluorescence intensity reaches the mean plus twice the standard deviation (b) of the pre-contact regime (before the tip touches the viron).

Fluorescence was acquired with a frame rate of $\approx 7.5 \mathrm{~Hz}$. Therefore, the data had to be interpolated to determine the fluorescence rising time. Interpolation, level finding, and time determination were performed with a custom written program shown under section 6.2. The results of the fluorescence rising time analysis are illustrated in figure 4.45. On a descriptive level, the WT fluorescence seems to rise indeed slightly faster. However, there is no statistically significant difference compared to the TS1 fluorescence rising time $(t(31.9)=1.2, p=0.22)$. 


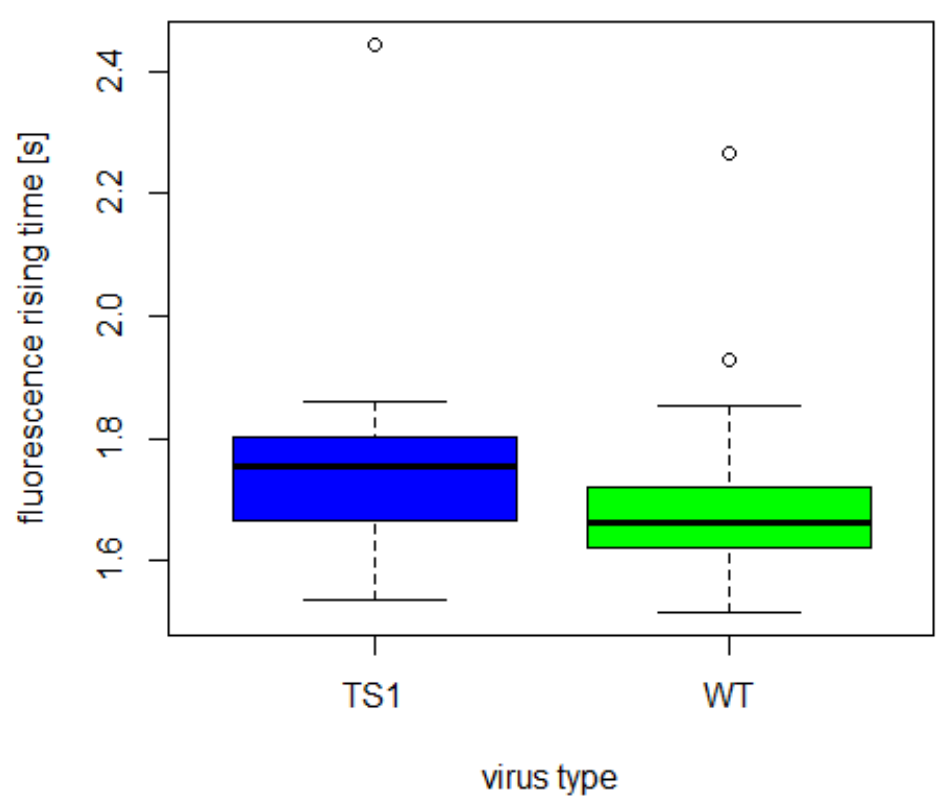

Figure 4.45: Boxplot, fluorescence rising time for WT and TS1. Statistically, there is no significant difference, but descriptively the WT signals rises slightly faster. The WT virus core may offer more dye binding places than the TS1 core.

If one plots the fluorescence rising time depending on the number of total emitted photons, it seems that more emitted photons correlate with a shorter fluorescence rising time. This would be consistent with the hypothesis mentioned above. The scatter plot can be found under the appendix section, fig. 6.1c.

\subsubsection{Discussion}

The human Adenovirus is only rendered infective after a maturation step during its assembly [43]. In contrast to the mature WT virus the immature TS1 phenotype is not infective. It remains in the endosome and never reaches the cell core, [46]. Using AFM in mechanical fatigue experiments has shown that after AFM induced shell disassembly, the remaining immature core and shell structures stay more condensed than the mature ones, [48]. These findings are consistent with mechanical effects of the maturation steps. If the step is missing, uncleaved protein precursors will remain, which stabilize the connection between capsids vertices and the viral 
genome. Furthermore, the viral DNA is condensed and stabilized by the protein precursors [43].

To complement the previous findings, the presented AFM-TIRFM setup was applied to identify DNA in the remaining core and to investigate spatial differences between the WT \& TS1 genome after induced shell disassembly. Here, the shell disassembly was induced by a single puncture (AFM tip). The subsequent spreading of the core was detected by fluorescence microscopy. The fluorescence labeling was facilitated by the use of a DNA specific dye present in the buffer, which becomes fluorescent when it binds to DNA (after shell rupture). The decompaction of the genome determines the spatial separation of the dye molecules and so the detected fluorescence spot size. Moreover, the offered dye binding places should scale (within limits) with the DNA decompactation level. Therefore, the fluorescence brightness and the fluorescence rising time could also deliver indicators for spatial genome differences after shell dissasembly.

These three parameters were measured and compared for the WT viruses and the TS1 viruses. To determine the offered binding places, the detected photons were counted cumulatively over a time of $80 \mathrm{~s}$ after shell disruption. This method also includes the contributions from dye molecules which bleach during the observations. The results show no significant differences between the WT and TS1 group. However, descriptively the WT group emits more photons. The fluorescence signal rising time also shows a tendency to be faster in case of the WT virons. These two parameters measure the virus core spread in an indirect way. A more direct parameter is the fluorescence spot size. Since the size of the remaining genome is close the diffraction limited spot size, the width (standard deviation) was derived from a 2D-Gauss fit to the intensity spots. The fluorescence spot width shows a significant higher value for the WT genome. These findings lead to the conclusion that the WT genome spreads more than the TS1 genome after shell disruption.

The measurements were performed with custom made glass-mica sample substrates since initial measurements revealed that proper virus immobilization could only be established on mica. The mica was cleaved directly before every experiment as thin as possible. Due to the manual procedure it can not be guaranteed that the mica thickness was exactly identical for every sample. This has to be taken into account since the optical properties of mica introduce a focal spot degradation which depends on its thickness. This could then effect the measured width of the 
viral cores. However, the influence on the width turned out to be small: $<\sim 10 \mathrm{~nm}$, (section 4.1.4.1). Because the average number of particles measured per sample substrate ranges around three, the effect should average out when comparing both groups. Furthermore, the width parameter is influenced by defocusing. In our experiments, the substrate surface was focused manually before each individual virus pushing so that the absolute minimal focus position in the longitudinal focal region might not have been reached in every case. The focal spot width changes over the visual depth of field by less than $\sim 10 \%$ (4.1.4.1). So, only a minor error on the width is expected. Again, the defocusing error is present in both viral groups. Hence, it is not expected to change the differences between both groups.

To sum it up, the AFM observations made by Ortega-Esteban et al. [48] could be completed by our AFM-TIRFM experiments. Strikingly, the WT genome could be shown to spread more than the TS1 genome by the additional use of fluorescence microscopy. Since the fluorescence detection is less invasive (non mechanical) than the detection by AFM imaging, we could show this effect also under more diffusive driven conditions. Due to the immobilization method (mica substrate $+N i^{2+}$ ), our experiments are not able to deliver information about the native diffusive behavior of the WT viral genome. We suggest that the DNA will be absorbed nearby the disrupted capsid, so that diffusion is inhibited and restricted. Moreover, the observed time evolution of the WT spot width (slight shrinking over time) indicates a DNA photocleavage by the fluorescent dye. Nevertheless, to our knowledge our experiments are the first that combined AFM and TIRFM to induce a virus shell disassembly followed by the specific labeling of the released genome after shell disruption in real time. Furthermore, we could show that the method is sensitive enough the detect the release of single pentons in the virus shell. Since the pentons are regarded as nucleation points for capsid disassembly, our work might serve as a basis for investigating capsid disassembly in more detail in the future.

Thus, this application of the combined AFM-TIRFM setup illustrates its potential of connecting mechanical interventions by AFM with specific non-invasive imaging by TIRFM. 


\section{Conclusion}

In this thesis a new method to combine AFM imaging and low force manipulation with time correlated fluorescence detection is presented. I developed a combined AFM-TIRFM setup with special emphasis on minimizing noise originating from the interaction of both microscope systems. Those include mechanical noise interactions and optical effects of the cantilever and tip. The capability to detect single dye molecules in close proximity of the AFM cantilever tip was demonstrated in experiments to characterize the fluorescence of labeled 70S ribosomes. The following conclusions could be drawn: the background light from the AFM cantilever and tip close to the ribosomes allows single molecule fluorescence detection only in the TIRFM configuration and not with the conventional epi-fluorescence configuration. For distances between tip and dye molecule of less than a few nanometer, the quenching potential of the AFM tip limits the detection of single dye labels. Analysis of bleaching times and signal to noise ratios show that we can detect single fluorescent molecules on the minute time scale at video frame rate. Alternatively, detection rates of up to $400 \mathrm{~Hz}$ are possible with observation times of several seconds.

To test the potential of the combined AFM-TIRFM setup, I applied it on the investigation of 3 completely biological different samples ranging from micrometer to nanometer scales: Parkinson's Disease (PD)-like cells, Adenoviruses, and actin binding proteins.

PD-like cell line: Three fluorescence sub-types of the PD-like cell line could be identified according to their LRRK2-distribution patterns by TIRFM. The mechanical properties for each of the sub-types could be quantified by AFM indentation experiments. As a striking result, only one sub-type showed mechanical properties (Young's modulus) similar to the control cells. Both other sub-types revealed a higher Young's modulus indicating a modified cellular organization leading to a 
higher cell stiffness. LRRK2 has been repeatedly identified as one of the inclusion components of Lewy bodies (cytosolic aggregations) in affected PD neurons. Interestingly, the mechanically unaffected sub-type is the one that indeed shows an aggregation pattern of the LRRK2-distribution. Whether the Lewy bodies are protective or toxic is still unclear [97], [98]. Therefore we speculate, that the aggregation of LRRK2 could prevent its significant involvement in a modification process affecting the cytoskeleton. The next goal is to find out when, and perspectivly how, cells can differentiate into the 3 sub-types. Fluorescence microscopy and mechanical testing could be one approach to follow these questions.

Adenoviruses: To study the mechanism of genome release of the Adenovirus a shell induced disassembly was applied on both a wild type (WT) and an genetically modified immature (TS1) virus. AFM was used to crack open the virus and genome spreading was observed in real time by TIRFM. The WT genome could be shown to spread out more and thus remains less condensed than the TS1 genome. To our knowledge, our experiments are the first facilitating and visualizing viral genome release with a combined AFM-TIRFM setup and have shown the inability of the non-infective TS1 virus to disperse its genome. Further experiments are planned to fluorescently label the various core proteins with different colors to identify the proteins that regulate the organization of the genome of the TS1 mutant.

Actin binding proteins: By direct imaging with AFM we could confirm that DBN-1 binds to actin filaments and bundles them in vitro. Prior to high resolution AFM imaging, TIRFM was used to localize the bundles and to identify actin. The presented results show that most of actin/DBN-1 bundles contain several filaments and that DBN-1 is localized along the filaments. Our findings are described in Butkevich et al. [50]. We speculate that DBN-1 cross-links actin filaments into bundles. This is consistent with the observation of micrometer sized loops formed by the actin bundles. Since the loop diameters are significant smaller than the persistence length of actin, DBN-1 is thought to stabilize the loops by cross-linking.

As an additional project I constructed and characterized a vertical optical tweezers instrument, which is described in Bodensiek et al. [58]. This technique was specifically developed to overcome the mechanical noise limitations of AFM. Indeed, the forces that can be controlled by the vertical optical tweezers are on the single pN level and an order of magnitude lower than in AFM. Its application in measuring 
the mechanics of living cells at very low forces and deformations is described by Nawaz et al. [89]. 



\section{Appendix}

\subsection{Mechanical unpacking of Adenovirus capsids: supplementary data}

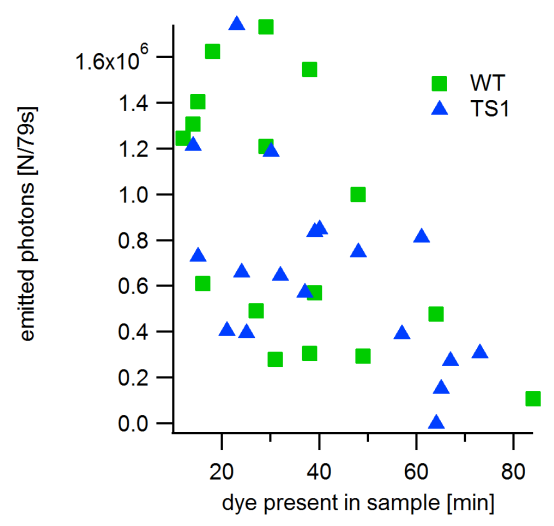

(a)

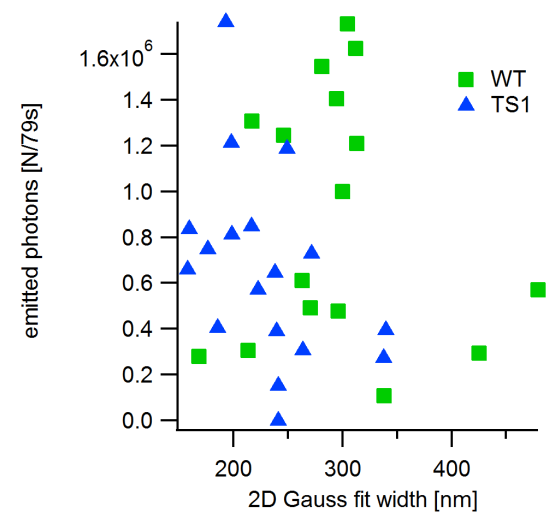

(b)

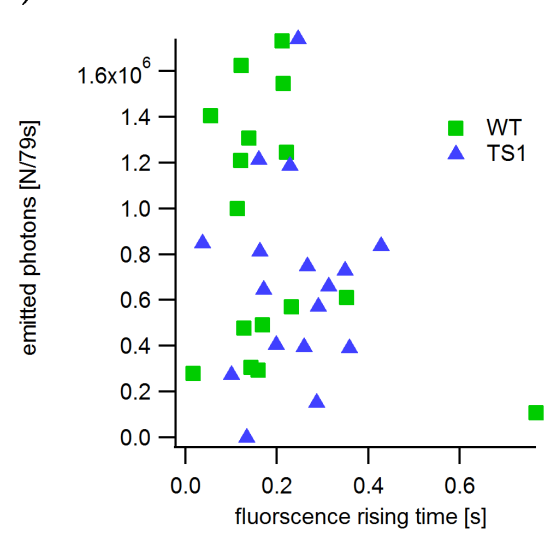

(c)

Figure 6.1: Scatter plot of emitted photons vs. sample age, width, fluorescence rising time. (a): Due to the experimental procedure (alignment of both microscopes, finding of viral particles), the individual particles are measured at different sample 'ages'. We plotted the total emitted photons as function of the 'age' (time after preparation). Clearly, there is temporal dependence of the emitted photons, the older a sample gets the less photons it emits. Since both virus groups are spread over comparable sample 'ages', we did not include this effect in our analysis. (b): There is no clear correlation between the total number of emitted photons and the measured width. Therefore, the higher average width of the WT group must originate from the spread behavior of the genome. (c): The fluorescence rising time shortens with the number of total emitted photons. This support our hypothesis that a more dispersed genome presents more binding places to the fluorescence dye. 


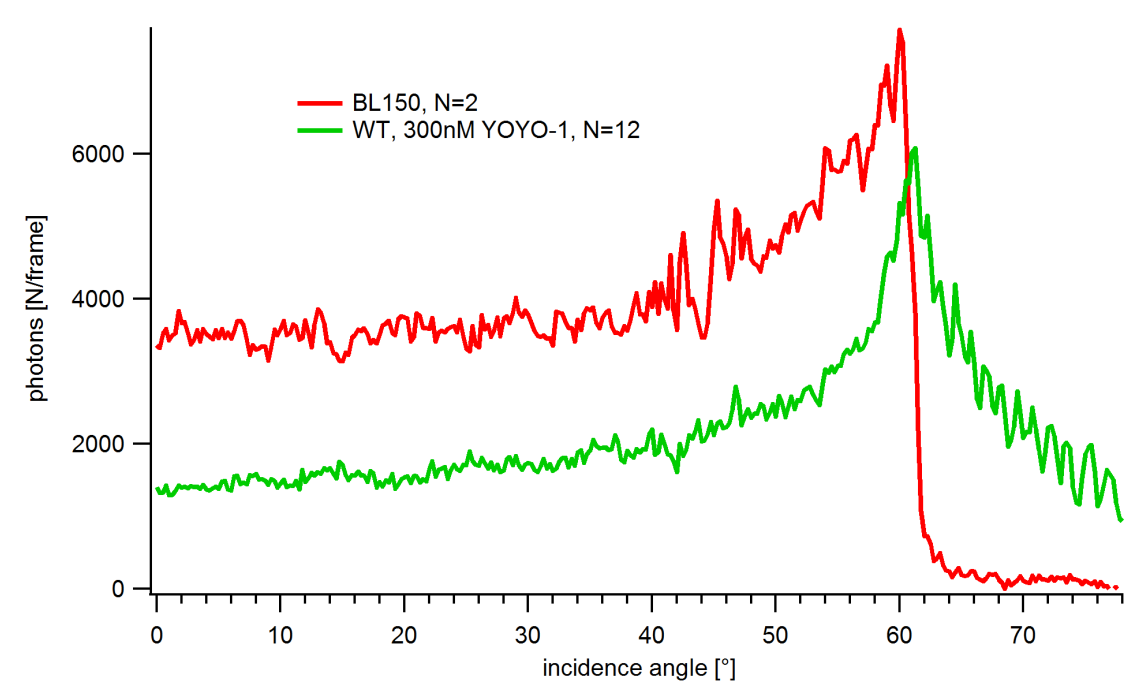

(a) Cantilever: BL-RC150-HW, (OLympus, Japan). V-shaped tip, Au coated

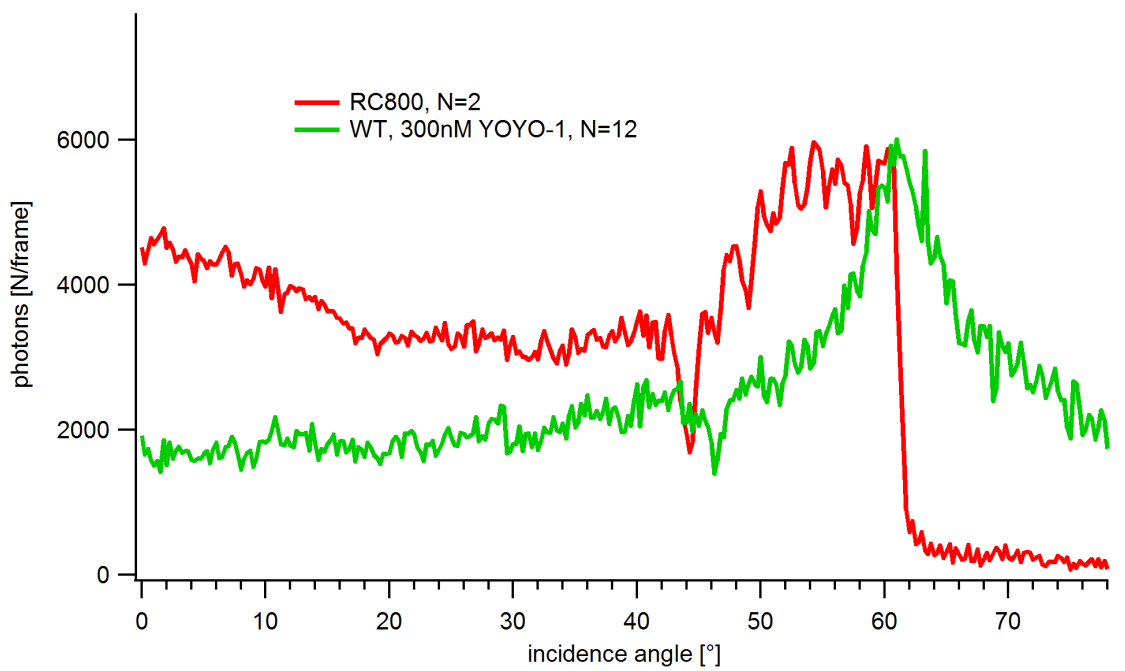

(b) Cantilever: OMCL-RC800PSA, (Olympus, Japan). Pyramidal tip, $\mathrm{Si}_{3} \mathrm{~N}_{4}$

Figure 6.2: Adenovirus genome fluorescence vs. background light from cantilevers from sub critical to super critical incidence angles. Even with the use of the appropriate high quality dichroic mirrors and bandpass filters the background signal from cantilever and tip can outshine fluorescent targets for illumination angles smaller than the critical angles of TIR. The fluorescence targets are single Adenovirus genomes. They become fluorescent when a DNA specific dye binds to them (YOYO-1 Iodide, Life Technologies, USA). For this experiment the capsids were ruptured by incubating the sample at a temperature of $65^{\circ} \mathrm{Cfor} 10 \mathrm{~min}$. Above the critical angles of TIR the cantilever tips are illuminated by the evanescent wave, which causes no extra background. Conclusively combined AFM-TIRFM operations for mechanical unpacking of Adenovirus capsids are only practical in the TIR range, above laser incidence angles of $61^{\circ}$. The tips are brought into contact with the Mica substrates with a force set point of $200 p N$. The exposure time of the EMCCD was $100 \mathrm{~ms}$, with a EM gain of $300 \%$. Laser intensity at $64^{\circ}$ is $0.34 \mu \mathrm{W} / \mu \mathrm{m}^{2}$, excitation beam diameter at $0^{\circ}$ is $82 \mu \mathrm{m}$. Used emission band pass filter: FF01-512/25-25, Semrock, USA. 


\subsection{Custom programs}


//Author: Kai Bodensiek, 2014

/Nersion: 0.4

\#pragma rtGlobals=1

\#include <All IP Procedures>

\#include <Image Saver>

\#include <AllStatsProcedures>

\#include <Waves Average>

//Main function, goes through the imagestack , fits the 2D gaussian, calculates positions and $/ /$ plots the results

Function PointsourceTracker(imageinstance)

//Array and variable declaration

Wave imageinstance

Variable stacksize, i, k, a=160, d, sx, sy, sz

Variable/G fitxo, fityo, fitywidth, fitxwidth, xywidth, nphotons, background, sem

stacksize $=$ dimsize $($ imageinstance, 2$)$

Make/O/N=(stacksize) xpositionwave, ypositionwave, zpositionwave, xywidthwave, photonswave

Make/O/N=(stacksize) xysemwave, timwave

Make/O/N=(stacksize-1) deltaxwave, deltaywave, deltaplanewave, deltaplanesemwave

Make/O/N=(2) cumlativephotonsmeanwidth

//Stack processing: point source positions and parameters in every frame

ImageGenerateROIMask orginalimage

for $(i=0 ; i<$ stacksize; $i+=1)$

ImageTransform/p=(i) getPlane imageinstance

CurveFit/NTHR=0/Q=1 Gauss2D M_ImagePlane /D/M=M_ROIMask

fitparameters(W_coef) I/Sub function call

xpositionwave[i]=fitxo*a

ypositionwave[i]=fityo*a

xywidth $=($ fitywidth+fitxwidth $) / 2$

xywidthwave $[i]=x y$ width*a

zpositionwave[i]=-1977+((23.051+xywidth*a)/0.00016429+1977^2)^(1/2)

nphotons $=0$

background $=0$

centroidpixelvalues(M_ImagePlane) I/Sub function call

sem $=0$

centroidsem() //Sub function call

xysemwave[i]=sem

photonswave $[i]=$ nphotons-background ${ }^{*}\left(\left(14^{*} x y \text { width }\right)^{\wedge} 2\right)$

endfor

//Parameters over the whole stack

cumlativephotonsmeanwidth[0]= sum (photonswave)

cumlativephotonsmeanwidth[1] = mean (xywidthwave) 
//Relative point source positions

for $(\mathrm{k}=0 ; \mathrm{k}<$ stacksize- $1 ; \mathrm{k}+=1)$

deltaxwave[k]=(xpositionwave[k+1]-xpositionwave[k])

deltaywave[k]=(ypositionwave[k+1]-ypositionwave[k])

deltaplanewave $[k]=\left(\text { deltaxwave }[k]^{\wedge} 2+\text { deltaywave }[k]^{\wedge} 2\right)^{\wedge}(1 / 2)$

deltaplanesemwave $[k]=\left(\left(x y s e m w a v e[k+1]^{\wedge} 2+x y \operatorname{semwave}[k]^{\wedge} 2\right)^{\wedge}(1 / 2)\right)$

endfor

//Absolute point source positions

sx=xpositionwave[0]

sy=ypositionwave[0]

sz=zpositionwave[0]

for $(d=0 ; d<$ stacksize; $d+=1)$

if(zpositionwave[d]<sz)

$\mathrm{sz}=z$ positionwave[d]

endif

endfor

for $(d=0 ; d<$ stacksize; $d+=1)$

xpositionwave[d]=xpositionwave[d]-sx

ypositionwave[d]=ypositionwave[d]-sy

zpositionwave[d]=zpositionwave[d]-sz

timwave $[\mathrm{d}]=\mathrm{d}^{*} 0.1$

endfor

//Data plotting

Edit

AppendToTable root:xpositionwave, ypositionwave, zpositionwave, xysemwave, deltaxwave, deltaywave, deltaplanewa AppendToTable deltaplanesemwave, xywidthwave, photonswave, timwave, cumlativephotonsmeanwidth

Display ypositionwave vs xpositionwave

ModifyGraph mode=3

ErrorBars ypositionwave $\mathrm{XY}$, wave $=($ xysemwave, xysemwave $)$, wave $=($ xysemwave, xysemwave $)$

ModifyGraph marker=16

ModifyGraph fSize(left)=13; DelayUpdate

Label left "IIZ14position in y [nm]"

ModifyGraph fSize=13;DelayUpdate

Label bottom "IIZ14position in x [nm]"

ModifyGraph zColor(ypositionwave)=\{timwave, , , , Rainbow256,0\}

ColorScale/C/N=text0/F=0/A=RC/E trace=ypositionwave;DelayUpdate

ColorScale/C/N=text0 "time [s]"

Display deltaywave vs deltaxwave

ModifyGraph mode=3

ErrorBars deltaywave $\mathrm{XY}$, wave=(deltaplanesemwave,deltaplanesemwave), wave=(deltaplanesemwave,deltaplanesemu ModifyGraph marker $=16$

ModifyGraph fSize(left)=13; DelayUpdate

Label left "IIZ14relative position in y [nm]"

ModifyGraph fSize=13;DelayUpdate

Label bottom "IIZ14relative position in x [nm]" 
Display xywidthwave vs timwave

Label left "IIZ14xy width [nm]"

Label bottom "IIZ14time [s]"

ModifyGraph mode=2, Isize $=2$

Display photonswave vs timwave

Label left "IIZ14photons [N]"

Label bottom "I|Z14time [s]"

ModifyGraph mode $=2$, Isize $=2$

concatenate\{xpositionwave,ypositionwave,zpositionwave\}, tripletwave

End

redimension

//Sub function, callded in every frame, delivers the fit parameters

Function fitparameters(Fitresults)

Wave Fitresults

variable/G fitxo,fityo,fitywidth, fitxwidth

fitxo=Fitresults [2]

fityo=Fitresults [4]

fitxwidth=Fitresults[3]

fitywidth=Fitresults [5]

End

//Sub function, callded in every frame, dcounts photons under the centroid minus the background

Function centroidpixelvalues(slice)

Wave slice

variable/G fitxo,fityo,xywidth,nphotons,background

Variable I,m

for $\left(I=\left(\right.\right.$ fityo $-7^{*} x y$ width $) ; \mid<\left(\right.$ fityo $+7^{*} x y$ width +1$)$; $\left.\mid+=1\right)$

for $\left(\mathrm{m}=\left(\right.\right.$ fitxo- $7^{*}$ xywidth $) ; \mathrm{m}<\left(\right.$ fitxo $+7^{*}$ xywidth +1$\left.) ; \mathrm{m}+=1\right)$

if(I ==(fityo-7*xywidth) \&\& m==(fitxo-7*xywidth)

background=slice[m] [l]

endif

if $\left(\mathrm{I}==\left(\right.\right.$ fityo $+7^{*} x y$ width $) \& \& \mathrm{~m}==\left(\right.$ fitxo $+7^{*}$ xywidth $\left.)\right)$

background+=slice[m] [l]

endif

if ( $==\left(\right.$ fityo- $7^{*}$ xywidth $) \& \& m==\left(\right.$ fitxo $+7^{*}$ xywidth $)$ )

background+=slice[m] [l]

endif

if $\left(\mathrm{I}==\left(\right.\right.$ fityo $+7^{*} x y$ width $) \& \& \mathrm{~m}==\left(\right.$ fitxo- $7^{*} x y$ width $\left.)\right)$

background+=slice[m] [l]

endif

nphotons $+=($ slice $[\mathrm{m}][\mathrm{l}])$

endfor

endfor 
16.07.2014

Procedure

background $=($ background $/ 4)$

End

//Sub function, callded in every frame, calculates the position standandard error of the mean

Function centroidsem()

Variable/G nphotons,background,xywidth,sem

Variable, $a=160$

sem $=\left(\left(x y w i d t{ }^{*} a\right)^{\wedge} 2 / \text { nphotons }+a^{\wedge} 2 /\left(12^{*} \text { nphotons }\right)+\left(8^{*} \text { pi }^{*}(x y \text { width*a } a)^{\wedge} 4^{*}(\text { background })^{\wedge} 2\right) /\left(a^{\wedge} 2^{*} n p h o t o n s^{\wedge} 2\right)\right)^{\wedge}(1 / 2)$

End 
//Author: Kai Bodensiek, 2014

INVersion: 0.2

\#lfdef ARrtGlobals

\#pragma rtGlobals=1

\#else

\#pragma rtGlobals $=3$

\#endif

\#include ":AsylumResearch:Code3D:Initialization"

\section{StartMeUp()}

\#pragma rtGlobals $=3$

\#pragma rtGlobals $=1$

\#include <All IP Procedures $>$

\#include < Image Saver $>$

\#include <AllStatsProcedures $>$

\#include <Waves Average $>$

//Main function, interpolates the fluorescence trace and findes the designated level

Function fluorescencerisingtime(ftrace,t)

Wave ftrace,t

Wave M_WaveStats

variable $\bar{i}, \mathbf{i} 2$,avg,sdev

string newWaveName=NameOfWave(ftrace)

print newWaveName

Make/O/N=18 precam

Interpolate $2 / \mathrm{T}=3 / \mathrm{N}=10000 / \mathrm{F}=0 / \mathrm{Y}=\mathrm{ftraceSS} \mathrm{t}$, ftrace

AppendToGraph/R ftraceSS

ModifyGraph mode(ftraceSS) $=2$,Isize (ftraceSS $)=3$; DelayUpdate

ModifyGraph rgb(ftraceSS $)=(0,52224,0)$

Showinfo

DoUpdate

for $(i=0 ; i<17 ; i+=1)$

precam[i]=ftrace[i]

endfor

WaveStats/Q precam

avg $=V \_a v g$

sdev $=\bar{V}$ sdev

print "avg=",avg, "sdev=",sdev

i2 $2=17^{*} 16$

do

i2+=1

If $\left(\right.$ ftraceSS $\left.[\mathrm{i} 2]>\left(\operatorname{avg}+\left(2^{*} \mathrm{Sdev}\right)\right)\right)$

print "point" ,i2 
16.07.2014

Cursor/P A,ftraceSs ,i2

break

endif

while $(\mathrm{i} 2<500)$

End
Procedure

2 


\section{List of abbreviations}

AFM Atomic Force Microscopy

TIRFM Total Internal Reflection Fluorescence Microscopy

MFD Mode Field Diameter

LD Laser Diode

DPSSL Diode Pumped Solid State Laser

CL Clear Aperture

DLS Defraction Limeted Spot

TIR Total Internal Reflection

PD Parkinson's Disease

IR Infrared

ATP Adenosine triphosphate

PSF Point spread function

FBS Fluorescence to Background Signal

Ad Adenovirus

DBN-1 DreBriN-like family homolog 1

Abp-1 Actin binding protein

mRNA messenger Ribonucleic acid

FWHM Full width at half maximum

TIRF Total Internal Reflection Fluorescence

LRRK2 Leucine-rich repeat kinase2 
GFP Green fluorescent protein

CNS Central nervous system

SEM Standard Error of the Mean

ANOVA Analysis of variance

EMCCD Electron Multiplying Charge Coupled Device

DNA Deoxyribonucleic acid

PSD Power Spectral Density

RMS Root mean square

FPI Fabry-Pérot-Interferometer

NA Numerical Aperture

BFP back-focal-plane

QPD quadrant photo diode

NIR near infrared

OEP objective entrance pupil

FP focal plane

PS polystyrene

FS focal spot

Si Silicon

SiN SiliconNitrate

CCD charge-coupled device

LED light-emitting diode 


\section{List of Tables}

4.1 Bleaching times of single labeled ribosome samples at different excitation intensities. . . . . . . . . . . . . 69

4.2 Bleaching times of mRNA labeled ribosomes vs. excitation intensity. 70

4.3 Post-hoc t tests between the three fluorescence sub-types. *: significant differences. . . . . . . . . . . . . . . . . 996 


\section{List of Figures}

2.1 AFM principle with its instrumental core components. . . . . . . 9

2.2 Fitting the cantilever model to the cantilevers PSD delivers its spring constant. . . . . . . . . . . . . . . . . 12

2.3 Objective type TIRFM. . . . . . . . . . . . . . . 13

2.4 Refraction and reflection of a plane wave at an interface. . . . . . . 14

2.5 Intensity decay of an evanescent wave. . . . . . . . . . . 16

2.6 Intensity $I_{p, s}(0)$ plotted versus $\theta_{1} \ldots \ldots \ldots \ldots$

2.7 Optical trapping principle. . . . . . . . . . . . . 20

2.8 PSD of a trapped bead $10 \mu m$ inside the sample chamber. . . . . . 22

2.9 Overall structure of the Adenovirus. . . . . . . . . . . . . 26

2.10 Fluctuations in height measured in preliminary AFM experiments on ribosomes. . . . . . . . . . . . . . . . . . . . 28

3.1 Principle of objective type TIR. . . . . . . . . . . . . . . 31

3.2 Functional layout of the combined AFM-TIRFM setup . . . . . . . 32

3.3 Spectral separation in the TIRFM. . . . . . . . . . 35

3.4 Rayleigh criteria. . . . . . . . . . . . . . 36

3.5 Optical design of the vertical optical trap. . . . . . . . . . . 41

3.6 Stress \& strain parameters . . . . . . . . . . . . . . . . 44

3.7 Elastic and viscouselastic material response. . . . . . . . . . . . . 44

3.8 The Standard Linear Solid model. . . . . . . . . . . . . . . . . . . 45

3.9 Sample and cantilever deformation during a force distance experiment with an spherical cantilever probe. . . . . . . . . . 46

3.10 AFM indentation. . . . . . . . . . . . . . . 47

3.11 Detection principle of fluorophore separations close to the diffraction limit. . . . . . . . . . . . . . . . . . . . 49

3.12 Refractive indexes in the sample support. . . . . . . . . . 52 
4.1 Mechanical realization of the central combination of both microscopes. 55

4.2 Mechanical realization of the combined AFM-TIRFM setup. . . . . 56

4.3 Instrument noise. . . . . . . . . . . . . . . . . . 57

4.4 Calibration curve for the galvo mirror system. . . . . . . . . . 59

4.5 Fluorescence microscopy localization accuracy with a mechanically decoupled camera. . . . . . . . . . . . . . 6 60

4.6 Capabilities to measure translations of point sources. . . . . . . . 61

4.7 Spot size of a $100 \mathrm{~nm}$ diameter fluorescent bead vs. z-focus position. 63

4.8 Three dimensional microscope drift within 30min. . . . . . . . . . 64

4.9 Measured evanescent field decay for different incidence angles. . . . 65

4.10 Intensity decay of $\sim 30$ single labeled ribosomes at varied laser excitation intensities. . . . . . . . . . . . . 68

4.11 Intensity decay of $\sim 400$ mRNA labeled (single label) ribosomes at varied laser excitation intensities. . . . . . . . . . . 69

4.12 Bleaching times vs. excitation intensity for peripheral and mRNA labels. . . . . . . . . . . . . . . . . 71

4.13 The FBS definition and its scaling with the excitation intensity. . . 72

$4.14 F B R$ vs. detection frequency. . . . . . . . . . . . . . 73

4.15 Cantilever-tip background and single labeled ribosomes vs. excitation mode. . . . . . . . . . . . . . . . . 74

4.16 BL 150 cantilever vs. single labeled ribosomes from sub-critical to super-critical incidence angles. . . . . . . . . . . . . 75

4.17 RC 800 cantilever vs. single labeled ribosomes from sub-critical to super-critical incidence angles. . . . . . . . . . . . . . 75

4.18 Quenching of a single labeled ribosome. . . . . . . . . . . . 76

4.19 Artifacts in force-clamp experiments with a low force set point. . . . 79

4.20 Drift during force-distance curves with a $40 s$ dwell on the surface. . 80

4.21 Adhesion forces and their influence on the cantilever. . . . . . . . 80

4.22 RMS force noise for different separation of cantilever an substrate. . 81

4.23 Mechanical design of the vertical optical trap. . . . . . . . . . 83

4.24 Trapping and detection performance. . . . . . . . . . . . . 85

4.25 The intrinsic force noise in AFM and optical trapping. . . . . . . . 86

4.26 Linearity of the trap stiffness in vertical direction. . . . . . . . . 88

4.27 Interference effect between bead and coverslip. . . . . . . . . . . . 89

4.28 Low force indentation. . . . . . . . . . . . . . . . 92 
4.29 Expression of eGFP-LRRK2 in H4 cell line. . . . . . . . . . . . . . 93

4.30 The distinct fluorescence sub-types. . . . . . . . . . . . . . . 94

4.31 Boxplots, main effect fluorescence sub-type. . . . . . . . . . 95

4.32 Both the fluorescence sub-type and the indentation indentation speed affected the measured Young's modulus. . . . . . . . . . . 96

4.33 Direct visualization of actin filament loops generated in the presence of DBN-1. . . . . . . . . . . . . . . . . 99

4.34 Detailed AFM imaging of actin fibers in presence and absence of

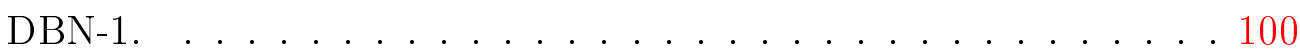

4.35 Mechanical unpacking of viral capsids. . . . . . . . . . . . . 102

4.36 Mechanical fatigue experiment. . . . . . . . . . . . . 103

4.37 Breaking force vs. spring constant. . . . . . . . . . . 105

4.38 AFM and TIRFM images before and after induced virus shell disruption. . . . . . . . . . . . . . 106

4.39 Virus capsid rupture and fluorescence increase. . . . . . . . . . . 107

4.40 Emitted photons after capsid rupture for the WT virus and TS1 mutant. . . . . . . . . . . . . . . . . . 108

4.41 Radial averaged intensity distribution of a single WT and a TS1 particle fitted with a Gauss function. . . . . . . . . . . . . . 109

4.42 Boxplots, average width of the intensity distributions from WT and TS1 cores after viral shell rupture. . . . . . . . . . . . . 110

4.43 Average width of WT and TS1 particles vs. time. . . . . . . . . . 111

4.44 Measuring the fluorescence rising time after capsid rupture. . . . . . 112

4.45 Boxplot, fluorescence rising time for WT and TS1. . . . . . . . . 113

6.1 Scatter plot of emitted photons vs. sample age, width, and fluorescence rising time. . . . . . . . . . . . . . . 120

6.2 Adenovirus genome fluorescence vs. background light from cantilevers from sub-critical to super-critical incidence angles. . . . . . 121 


\section{Bibliography}

[1] G. Binnig and C. F. Quate. Atomic force microscope. Physical Review Letters, 56(9):930-933, Mar 1986.

[2] R. E. Mahaffy, S. Park, E. Gerde, J. Käs, and C. K. Shih. Quantitative analysis of the viscoelastic properties of thin regions of fibroblasts using atomic force microscopy. Biophys J, 86(3):1777-1793, Mar 2004.

[3] Yu G. Kuznetsov and Alexander McPherson. Atomic force microscopy investigation of turnip yellow mosaic virus capsid disruption and rna extrusion. Virology, 352(2):329-337, Sep 2006.

[4] Sanja Trajkovic, Xiaoning Zhang, Sylvia Daunert, and Yuguang Cai. Atomic force microscopy study of the conformational change in immobilized calmodulin. Langmuir, 27(17):10793-10799, Sep 2011.

[5] N A Burnham, X Chen, C S Hodges, G A Matei, E J Thoreson, C J Roberts, M C Davies, and S J B Tendler. Comparison of calibration methods for atomic-force microscopy cantilevers. Nanotechnology, 14(1):1-6, Jan 2003.

[6] H J Butt and M Jaschke. Calculation of thermal noise in atomic force microscopy. Nanotechnology, 6(1):1-7, Jan 1995.

[7] Frederick Gittes and Christoph F. Schmidt. Chapter 8 signals and noise in micromechanical measurements. In Michael P. Sheetz, editor, Methods in Cell Biology, volume Volume 55, pages 129-156. Academic Press, 1997.

[8] R Proksch, T E Schaeffer, J P Cleveland, R C Callahan, and M B Viani. Finite optical spot size and position corrections in thermal spring constant calibration. Nanotechnology, 15(9):1344-1350, Sep 2004.

[9] E. J. AMBROSE. A surface contact microscope for the study of cell movements. Nature, 178(4543):1194-1194, Nov 1956. 
[10] D. Axelrod. Topics in Fluorescence Spectroscopy, Vol. 3: Biochemical Applications, chapter \%. Springer, 1992.

[11] Hecht. Optik. Oldenbourg, 2009.

[12] Lukas Novotny and Bert Hecht. Principles of Nano-Optics. Cambridge University Press, 2012.

[13] Bergmann,Ludwig / Schaefer,Clemens: Lehrbuch Der Experimentalphysik: Optik - Wellen- Und Teilchenoptik: Vol 3. Walter de Gruyter \& Co, 2004.

[14] Anselmetti D. Frey H G, Paskarbeit J. Tip-enhanced single molecule fluorescence near-field microscopy in aqueous environment. Appl Phys Lett, 94(24), 2009 .

[15] Yasushi Inouye Norihiko Hayazawa. Metallized tip amplification of near-field raman scattering. Optics Communications, 183, 2000.

[16] Pfeiffer Brixner, Aeschlimann. Ultraschnelle nanooptik. Physik Journal, 13:35-41, 2014.

[17] J Azoulay, A Debarre, A Richard, and P Tchenio. Quenching and enhancement of single-molecule fluorescence under metallic and dielectric tips. Europhysics Letters (EPL), 51(4):374-380, Aug 2000.

[18] Nader A. Issa and Reinhard Guckenberger. Fluorescence near metal tips: The roles of energy transfer and surface plasmon polaritons. Opt Express, 15(19):12131-12144, Sep 2007.

[19] B. Persson and N. Lang. Electron-hole-pair quenching of excited states near a metal. Phys. Rev. B, 26(10):5409-5415, Nov 1982.

[20] Keir C. Neuman and Steven M. Block. Optical trapping. Review of Scientific Instruments, 75(9):2787, 2004.

[21] A. Ashkin. Forces of a single-beam gradient laser trap on a dielectric sphere in the ray optics regime. Biophys J, 61(2):569-582, Feb 1992.

[22] Christoph F. Schmidt Frederick Gittes. Thermal noise limitations on micromechanical experiments. Eur Biophys J, 27:75-81, 1998.

[23] F. Gittes and C. F. Schmidt. Signals and noise in micromechanical measurements. Methods Cell Biol, 55:129-156, 1998. 
[24] Miriam W. Allersma, Frederick Gittes, Michael J. deCastro, Russell J. Stewart, and Christoph F. Schmidt. Two-dimensional tracking of ncd motility by back focal plane interferometry. Biophysical Journal, 74(2):1074-1085, Feb 1998.

[25] Erik Schaeffer, Simon F. Norrelykke, and Jonathon Howard. Surface forces and drag coefficients of microspheres near a plane surface measured with optical tweezers. Langmuir, 23(7):3654-3665, Mar 2007.

[26] Bahaa E. A. Saleh and Malvin Carl Teich. Grundlagen Der Photonik (German Edition). Wiley-VCH Verlag GmbH, 2008.

[27] A. Pralle, M. Prummer, E. L. Florin, E. H. Stelzer, and J. K. Hörber. Threedimensional high-resolution particle tracking for optical tweezers by forward scattered light. Microsc Res Tech, 44(5):378-386, Mar 1999.

[28] Ignacio F. Mata, Harvey Checkoway, Carolyn M. Hutter, Ali Samii, John W. Roberts, Hojoong M. Kim, Pinky Agarwal, Victoria Alvarez, Renee Ribacoba, Pau Pastor, Oswaldo Lorenzo-Betancor, Jon Infante, María Sierra, Pilar Gómez-Garre, Pablo Mir, Beate Ritz, Shannon L. Rhodes, Amy Colcher, Vivianna Van Deerlin, Kathryn A. Chung, Joseph F. Quinn, Dora Yearout, Erica Martinez, Federico M. Farin, Jia Y. Wan, Karen L. Edwards, and Cyrus P. Zabetian. Common variation in the lrrk2 gene is a risk factor for parkinson's disease. Mov Disord, 27(14):1822-1825, Dec 2012.

[29] Patricia Guerreiro. The role of LRRK2 in Parkinson's disease: from function to dysfunction. PhD thesis, Universidade de Lisboa, 2014.

[30] Loukia Parisiadou and Huaibin Cai. Lrrk2 function on actin and microtubule dynamics in parkinson disease. Commun Integr Biol, 3(5):396-400, Sep 2010.

[31] Loukia Parisiadou, Chengsong Xie, Hyun Jin Cho, Xian Lin, Xing-Long Gu, Cai-Xia Long, Evy Lobbestael, Veerle Baekelandt, Jean-Marc Taymans, Lixin Sun, and Huaibin Cai. Phosphorylation of ezrin/radixin/moesin proteins by lrrk2 promotes the rearrangement of actin cytoskeleton in neuronal morphogenesis. J Neurosci, 29(44):13971-13980, Nov 2009.

[32] Andrea Meixner, Karsten Boldt, Marleen Van Troys, Manor Askenazi, Christian J. Gloeckner, Matthias Bauer, Jarrod A. Marto, Christophe Ampe, Norbert Kinkl, and Marius Ueffing. A quick screen for lrrk2 interaction partners- 
leucine-rich repeat kinase 2 is involved in actin cytoskeleton dynamics. Mol Cell Proteomics, 10(1):M110.001172, Jan 2011.

[33] Guoxiang Liu, Leonardo Aliaga, and Huaibin Cai. Alpha-synuclein, lrrk2 and their interplay in parkinson's disease. Future Neurol, 7(2):145-153, Mar 2012.

[34] Manfred Radmacher. Studying the mechanics of cellular processes by atomic force microscopy. Methods Cell Biol, 83:347-372, 2007.

[35] S SURESH. Biomechanics and biophysics of cancer cells. Acta Biomaterialia, 3(4):413-438, Jul 2007.

[36] Yun-Bi Lu, Kristian Franze, Gerald Seifert, Christian Steinhäuser, Frank Kirchhoff, Hartwig Wolburg, Jochen Guck, Paul Janmey, Er-Qing Wei, Josef Käs, and Andreas Reichenbach. Viscoelastic properties of individual glial cells and neurons in the cns. Proc Natl Acad Sci US A, 103(47):1775917764, Nov 2006.

[37] Sang-Myung Lee, Thi-Huong Nguyen, Kyounghwan Na, Il-Joo Cho, Dong Ho Woo, Jae-Eung Oh, C Justin Lee, and Eui-Sung Yoon. Nanomechanical measurement of astrocyte stiffness correlated with cytoskeletal maturation. J Biomed Mater Res A, Mar 2014.

[38] J N Glasgow, M Everts, and D T Curiel. Transductional targeting of adenovirus vectors for gene therapy. Cancer Gene Therapy, 13(9):830-844, Jan 2006 .

[39] H. Liu, L. Jin, S. B. S. Koh, I. Atanasov, S. Schein, L. Wu, and Z. H. Zhou. Atomic structure of human adenovirus by cryo-em reveals interactions among protein networks. Science, 329(5995):1038-1043, Aug 2010.

[40] Michael A Kennedy and Robin J Parks. Adenovirus virion stability and the viral genome: Size matters. Molecular Therapy, 17(10):1664-1666, Oct 2009.

[41] Yvette Chardonnet and Samuel Dales. Early events in the interaction of adenoviruses with hela cells. Virology, 40(3):462-477, Mar 1970.

[42] M. Y. Nakano, K. Boucke, M. Suomalainen, R. P. Stidwill, and U. F. Greber. The first step of adenovirus type 2 disassembly occurs at the cell surface, 
independently of endocytosis and escape to the cytosol. Journal of Virology, 74(15):7085-7095, Aug 2000.

[43] A. J. Perez-Berna, A. Ortega-Esteban, R. Menendez-Conejero, D. C. Winkler, M. Menendez, A. C. Steven, S. J. Flint, P. J. de Pablo, and C. San Martin. The role of capsid maturation on adenovirus priming for sequential uncoating. Journal of Biological Chemistry, 287(37):31582-31595, Sep 2012.

[44] K. Wang, T. Guan, D. A. Cheresh, and G. R. Nemerow. Regulation of adenovirus membrane penetration by the cytoplasmic tail of integrin beta 5 . Journal of Virology, 74(6):2731-2739, Mar 2000.

[45] Sten Strunze, Martin F. Engelke, I-Hsuan Wang, Daniel Puntener, Karin Boucke, Sibylle Schleich, Michael Way, Philipp Schoenenberger, Christoph J. Burckhardt, and Urs F. Greber. Kinesin-1-mediated capsid disassembly and disruption of the nuclear pore complex promote virus infection. Cell Host $\mathcal{G}$ Microbe, 10(3):210-223, Sep 2011.

[46] U. F. Greber, P. Webster, J. Weber, and A. Helenius. The role of the adenovirus protease on virus entry into cells. EMBO J, 15(8):1766-1777, Apr 1996.

[47] Oliver Meier and Urs F. Greber. Adenovirus endocytosis. The Journal of Gene Medicine, 6(S1):S152-S163, Feb 2004.

[48] A. Ortega-Esteban, A. J. Perez-Berna, R. Menendez-Conejero, S. J. Flint, C. San Martin, and P. J. de Pablo. Monitoring dynamics of human adenovirus disassembly induced by mechanical fatigue. Sci. Rep., 3, Mar 2013.

[49] Sabine Connert, Simone Wienand, Cora Thiel, Maria Krikunova, Nataliya Glyvuk, Yaroslav Tsytsyura, Denise Hilfiker-Kleiner, Jörg W. Bartsch, Jürgen Klingauf, and Jürgen Wienands. Sh3p7/mabp1 deficiency leads to tissue and behavioral abnormalities and impaired vesicle transport. EMBO J, 25(8):1611-1622, Apr 2006.

[50] Butkevich E. Bodensiek K. von Roden K. Schaap I. Majoul I. Schmidt C. and Klopfenstein D. Drebrin-like protein 1 is a novel sarcomere component which stabilizes actin filaments during muscle contraction. Under review at NCB, 2014. 
[51] Erin E. Wilson and Michael W. Davidson Rudi Rottenfusser. Education in microscopy and digital imaging. Technical report, ZEISS Microscopy Online Campus, 2012.

[52] M. K. Cheezum, W. F. Walker, and W. H. Guilford. Quantitative comparison of algorithms for tracking single fluorescent particles. Biophys J, 81(4):23782388 , Oct 2001.

[53] Bo Zhang, Josiane Zerubia, and Jean-Christophe Olivo-Marin. Gaussian approximations of fluorescence microscope point-spread function models. Appl Opt, 46(10):1819-1829, Apr 2007.

[54] Russell E. Thompson, Daniel R. Larson, and Watt W. Webb. Precise nanometer localization analysis for individual fluorescent probes. Biophys J, 82(5):2775-2783, May 2002.

[55] Paul R. Selvin, Tyler Lougheed, Melinda Tonks Hoffman, Hyokeun Park, Hamza Balci, Benjamin H. Blehm, and Erdal Toprak. Fluorescence imaging with one-nanometer accuracy (fiona). CSH Protoc, 2007:Fluorescence Imaging with One Nanometer Accuracy, 2007.

[56] Joerg Enderlein, Erdal Toprak, and Paul R. Selvin. Polarization effect on position accuracy of fluorophore localization. Opt Express, 14(18):8111-8120, Sep 2006.

[57] Frédéric Eghiaian and Iwan A.T. Schaap chapter 6. Single Molecule Enzymology: Methods and Protocols (Methods in Molecular Biology). Humana Press, 2011.

[58] Kai Bodensiek, Weixing Li, Paula Sanchez, Schanila Nawaz, and Iwan A. T. Schaap. A high-speed vertical optical trap for the mechanical testing of living cells at piconewton forces. Review of Scientific Instruments, 84(11):113707, 2013 .

[59] H. Liang, K.T. Vu, P. Krishnan, T.C. Trang, D. Shin, S. Kimel, and M.W. Berns. Wavelength dependence of cell cloning efficiency after optical trapping. Biophysical Journal, 70(3):1529-1533, Mar 1996.

[60] K. Svoboda and S. M. Block. Biological applications of optical forces. Annu Rev Biophys Biomol Struct, 23:247-285, 1994. 
[61] Christopher Deufel and Michelle D. Wang. Detection of forces and displacements along the axial direction in an optical trap. Biophys J, 90(2):657-667, Jan 2006.

[62] Jakob Kisbye Dreyer, Kirstine Berg-Soerensen, and Lene Oddershede. Improved axial position detection in optical tweezers measurements. Appl. Opt., 43(10):1991, 2004.

[63] Hamid Ladjal, Jean-Luc Hanus, Anand Pillarisetti, Carol Keefer, Antoine Ferreira, and Jaydev P. Desai. Atomic force microscopy-based single-cell indentation: Experimentation and finite element simulation. IEEE/RSJ International Conference on Intelligent Robots and Systems, 2009.

[64] R. E. Mahaffy, C. K. Shih, F. C. MacKintosh, and J. Käs. Scanning probebased frequency-dependent microrheology of polymer gels and biological cells. Phys Rev Lett, 85(4):880-883, Jul 2000.

[65] Valentin L. Popov. Contact mechanics and friction. Springer, 2010.

[66] E. A-Hassan, W. F. Heinz, M. D. Antonik, N. P. D'Costa, S. Nageswaran, C. A. Schoenenberger, and J. H. Hoh. Relative microelastic mapping of living cells by atomic force microscopy. Biophys $J, 74(3): 1564-1578$, Mar 1998.

[67] Anthony C. Fischer-Cripps. Introduction to Contact Mechanics (Mechanical Engineering Series). Springer, 2007.

[68] Wolfgang Singer, Michael Totzeck, and Herbert Gross. Handbook of Optical Systems, Physical Image Formation (Volume 2). Wiley-VCH, 2005.

[69] D. J. Müller, M. Amrein, and A. Engel. Adsorption of biological molecules to a solid support for scanning probe microscopy. J Struct Biol, 119(2):172-188, Jul 1997.

[70] The olympus bio-lever- the first small, low noise cantilever for ac in fluid and force measurements for soft samples. Technical report, Asylum Research, 2010 .

[71] Leica-Microsystems. Leica dvm - 3d visualisation. Technical report, 2008.

[72] S. Pedersen. Escherichia coli ribosomes translate in vivo with variable rate. EMBO J, 3(12):2895-2898, Dec 1984. 
[73] Stefan Wennmalm and Rudolf Rigler. On death numbers and survival times of single dye molecules. The Journal of Physical Chemistry B, 103(13):25162519, Apr 1999.

[74] C. Kanony, B. Akerman, and E. Tuite. Photobleaching of asymmetric cyanines used for fluorescence imaging of single dna molecules. J Am Chem Soc, 123(33):7985-7995, Aug 2001.

[75] Nina Mauser and Achim Hartschuh. Tip-enhanced near-field optical microscopy. Chem Soc Rev, 43(4):1248-1262, Feb 2014.

[76] D. J. Müller, D. Fotiadis, S. Scheuring, S. A. Müller, and A. Engel. Electrostatically balanced subnanometer imaging of biological specimens by atomic force microscope. Biophys J, 76(2):1101-1111, Feb 1999.

[77] G. Dietler B. Cappella. Force-distance curves by atomic force microscopy. Surface Science Reports, 34:1-104, 1999.

[78] M. Kappl H.-J. Butt, B. Cappella. Force measurements with the atomic force microscope: Technique, interpretation and applications. Surface Science Reports, 59:1-152, 2005.

[79] Peter C. Seitz, Ernst H. K. Stelzer, and Alexander Rohrbach. Interferometric tracking of optically trapped probes behind structured surfaces: a phase correction method. Appl. Opt., 45(28):7309, 2006.

[80] S. Nader S. Reihani and Lene B. Oddershede. Optimizing immersion media refractive index improves optical trapping by compensating spherical aberrations. Optics Letters, 32(14):1998, 2007.

[81] Keir C. Neuman, Elio A. Abbondanzieri, and Steven M. Block. Measurement of the effective focal shift in an optical trap. Optics Letters, 30(11):1318, Jun 2005 .

[82] Volker Bormuth, Anita Jannasch, Marcel Ander, Carlos M. van Kats, Alfons van Blaaderen, Jonathon Howard, and Erik Schäffer. Optical trapping of coated microspheres. Optics Express, 16(18):13831, Sep 2008.

[83] U. F. Keyser, J. van der Does, C. Dekker, and N. H. Dekker. Optical tweezers for force measurements on DNA in nanopores. Review of Scientific Instruments, 77(10):105105, 2006. 
[84] Sebastian Knust, Andre Spiering, Henning Vieker, Andre Beyer, Armin Gölzhäuser, Katja Tönsing, Andy Sischka, and Dario Anselmetti. Videobased and interference-free axial force detection and analysis for optical tweezers. Review of Scientific Instruments, 83(10):103704, 2012.

[85] Masahiko Daimon and Akira Masumura. Measurement of the refractive index of distilled water from the near-infrared region to the ultraviolet region. Appl. Opt., 46(18):3811, 2007.

[86] Anita Jannasch, Mohammed Mahamdeh, and Erik Schäffer. Inertial effects of a small brownian particle cause a colored power spectral density of thermal noise. Physical Review Letters, 107(22), Nov 2011.

[87] K. Svoboda and S. M. Block. Force and velocity measured for single kinesin molecules. Cell, 77(5):773-784, Jun 1994.

[88] Jin-Der Wen, Laura Lancaster, Courtney Hodges, Ana-Carolina Zeri, Shige H. Yoshimura, Harry F. Noller, Carlos Bustamante, and Ignacio Tinoco. Following translation by single ribosomes one codon at a time. Nature, 452(7187):598-603, Apr 2008.

[89] Schanila Nawaz, Paula Sanchez, Kai Bodensiek, Sai Li, Mikael Simons, and Iwan A. T. Schaap. Cell visco-elasticity measured with afm and optical trapping at sub-micrometer deformations. PLoS ONE, 7(9):e45297, Sep 2012.

[90] Frank Gillardon. Leucine-rich repeat kinase 2 phosphorylates brain tubulinbeta isoforms and modulates microtubule stability-a point of convergence in parkinsonian neurodegeneration? J Neurochem, 110(5):1514-1522, Sep 2009 .

[91] Bernard M H. Law, Victoria A. Spain, Veronica H L. Leinster, Ruth Chia, Alexandra Beilina, Hyun J. Cho, Jean-Marc Taymans, Mary K. Urban, Rosa M. Sancho, Marian Blanca Ramírez, Saskia Biskup, Veerle Baekelandt, Huaibin Cai, Mark R. Cookson, Daniel C. Berwick, and Kirsten Harvey. A direct interaction between leucine-rich repeat kinase 2 and specific betatubulin isoforms regulates tubulin acetylation. J Biol Chem, 289(2):895-908, Jan 2014.

[92] Payal N. Gandhi, Xinglong Wang, Xiongwei Zhu, Shu G. Chen, and Amy L. Wilson-Delfosse. The roc domain of leucine-rich repeat kinase 2 is sufficient 
for interaction with microtubules. J Neurosci Res, 86(8):1711-1720, Jun 2008.

[93] Mareike Caesar, Susanne Zach, Coby B. Carlson, Kathrin Brockmann, Thomas Gasser, and Frank Gillardon. Leucine-rich repeat kinase 2 functionally interacts with microtubules and kinase-dependently modulates cell migration. Neurobiol Dis, 54:280-288, Jun 2013.

[94] F. Gillardon. Interaction of elongation factor 1-alpha with leucine-rich repeat kinase 2 impairs kinase activity and microtubule bundling in vitro. Neuroscience, 163(2):533-539, Oct 2009.

[95] Mahaboobi Jaleel, R Jeremy Nichols, Maria Deak, David G. Campbell, Frank Gillardon, Axel Knebel, and Dario R. Alessi. Lrrk2 phosphorylates moesin at threonine-558: characterization of how parkinson's disease mutants affect kinase activity. Biochem J, 405(2):307-317, Jul 2007.

[96] Patrícia Silva Guerreiro, Yue Huang, Amanda Gysbers, Danni Cheng, Wei Ping Gai, Tiago Fleming Outeiro, and Glenda Margaret Halliday. Lrrk2 interactions with alpha-synuclein-synuclein in parkinson's disease brains and in cell models. J Mol Med (Berl), 91(4):513-522, Apr 2013.

[97] James T. Lee, Tiffany C. Wheeler, Lian Li, and Lih-Shen Chin. Ubiquitination of alpha-synuclein by siah-1 promotes alpha-synuclein aggregation and apoptotic cell death. Hum Mol Genet, 17(6):906-917, Mar 2008.

[98] Mikiei Tanaka, Yong Man Kim, Gwang Lee, Eunsung Junn, Takeshi Iwatsubo, and M Maral Mouradian. Aggresomes formed by alpha-synuclein and synphilin-1 are cytoprotective. J Biol Chem, 279(6):4625-4631, Feb 2004.

[99] Joost van Mameren, Karen C. Vermeulen, Fred Gittes, and Christoph F. Schmidt. Leveraging single protein polymers to measure flexural rigidity. $J$ Phys Chem B, 113(12):3837-3844, Mar 2009.

[100] W. M. Gelbart and C. M. Knobler. Virology: Pressurized viruses. Science, 323(5922):1682-1683, Mar 2009.

[101] B. Akerman and E. Tuite. Single- and double-strand photocleavage of dna by yo, yoyo and toto. Nucleic Acids Res, 24(6):1080-1090, Mar 1996. 